\title{
DESIGN AND CHARACTERIZATION OF A CERVICAL PHANTOM MODEL FOR
} BIRTH SIMULATION TRAINING

\author{
by \\ Michelle Jennifer Luk, B.Eng \\ Ryerson University \\ 2016
}

\begin{abstract}
A thesis presented to Ryerson University
in partial fulfillment of the requirements for the degree of Master of Applied Science in the Program of

Electrical and Computer Engineering
\end{abstract}

Toronto, Ontario Canada, 2016

(C) Michelle Luk 2016 


\section{AUTHOR'S DECLARATION FOR ELECTRONIC SUBMISSION OF A THESIS}

I hereby declare that I am the sole author of this thesis. This is a true copy of the thesis, including any required final revisions, as accepted by my examiners.

I authorize Ryerson University to lend this thesis to other institutions or individuals for the purpose of scholarly research

I further authorize Ryerson University to reproduce this thesis by photocopying or by other means, in total or in part, at the request of other institutions or individuals for the purpose of scholarly research.

I understand that my thesis may be made electronically available to the public. 


\title{
Design and Characterization of a Cervical Phantom Model for Birth Simulation Training
}

Michelle Luk

Master of Applied Science, 2016

Electrical and Computer Engineering

Ryerson University

\begin{abstract}
In the midwifery and obstetrics, accurate diagnosis of labour progression is critical to ensure maternal and fetal wellbeing. In light of this, simulation systems have been designed to train practitioners. However, existing simulators lack a dynamic cervix to simulate the varying compliance of cervical ripening. In this thesis, characteristics of ex vivo human cervical tissue were studied and a pneumatically controlled compliant cervix phantom was designed. Cervical tissue exhibited strain of 0.50 for an applied stress of $325 \mathrm{kPa}$. The phantom demonstrated $35 \%$ effacement, $1 \mathrm{~cm}$ dilation and was capable of becoming 22 times softer. At maximum vacuum pressure, strain of 0.23 was achieved for an externally applied stress of $419 \mathrm{kPa}$. At atmospheric pressure, strain of 0.50 was achieved for an applied stress of $117 \mathrm{kPa}$. Results yield a deeper understanding of cervix characteristics and establishes the groundwork for the design of a dynamic human birth simulation training system.
\end{abstract}




\section{Acknowledgments}

I would first like to thank my supervisor Dr. James Andrew Smith for his continual support, encouragement and insight. He consistently allowed this paper to be my own work, but steered me in the right the direction whenever he thought I needed it.

I would also like to thank the numerous colleagues and staff members at both Ryerson University and McMaster University for their assistance. Particularly, thanks to Dr. Derek Lobb and the staff of the Anatomical Pathology lab at McMaster Health Sciences Centre for providing us with cervix tissue samples and insights into the biology of the cervix. Thanks to Jim Koch and Nripendra Malhotra in the Electrical Engineering Department of Ryerson University for their help in the mechanical workshop and access to the 3D printer. Thanks to Jung Kim, Andrew Christie and Sulmaz Zahedi for their assistance with the experimental setups and coding.

Finally, I must express my very profound gratitude to my parents for providing me with unfailing support and continuous encouragement throughout my years of study and through the process of researching and writing this thesis. This accomplishment would not have been possible without them. Thank you. 


\section{Table of Contents}

AUTHOR'S DECLARATION FOR ELECTRONIC SUBMISSION OF A THESIS ............ ii

Abstract.....................................................................................................................................

Acknowledgments ........................................................................................................................... iv

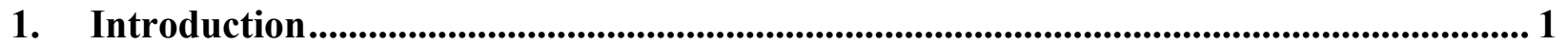

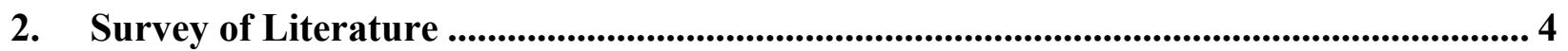

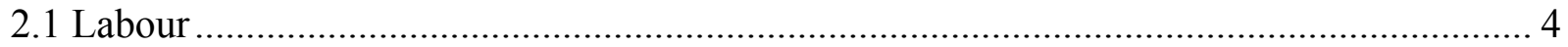

2.1.1 Stages of Labour \& the Role of the Cervix ...........................................................................

2.1.2 Clinical Diagnosis of Labour Stages...............................................................................6

2.1.3 Induction of Labour...............................................................................................

2.2 Simulation Training Systems .................................................................................. 9

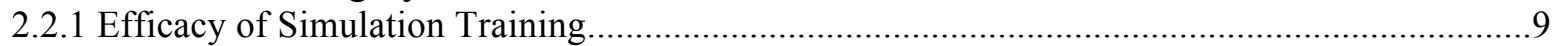

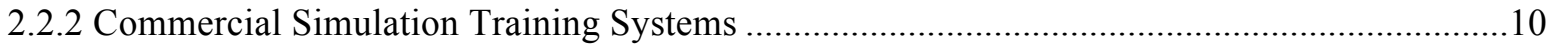

2.2.3 Experimental Cervical Models.............................................................................................11

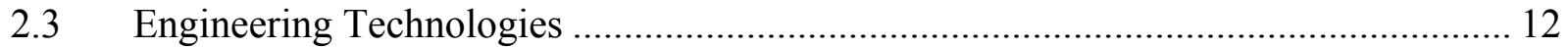

2.3.1 Soft Robotics and Particle Jamming …………………..................................................12

2.3.2 3D Printing Technology ……………………………………………………………...

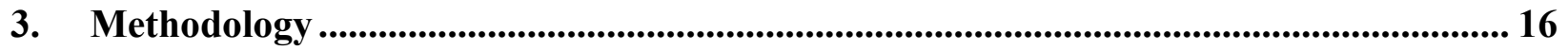

3.1 Ex Vivo Cervical Tissue Experimentation ............................................................ 16

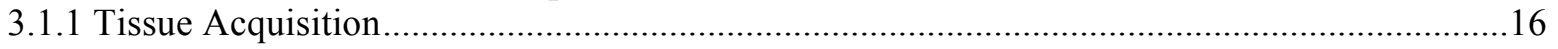

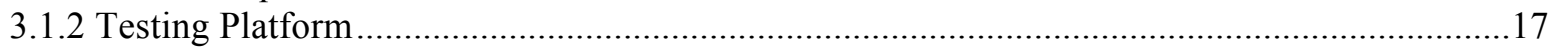

3.1.3 Tissue Characterization .................................................................................................

3.2 Design and Characterization of the Cervical Phantom ............................................... 21

3.2.1 Design of the Phantom ................................................................................................2

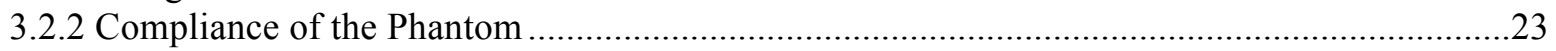

3.2.3 Validation of the Phantom through Tactile Psychophysics Experiments ..................................24

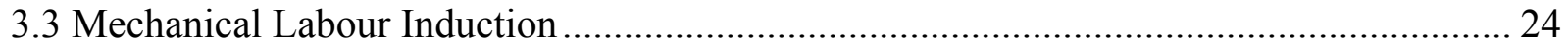

3.3.1 Optical Measurement using Kinect Cameras .........................................................................2

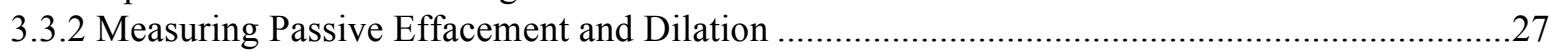

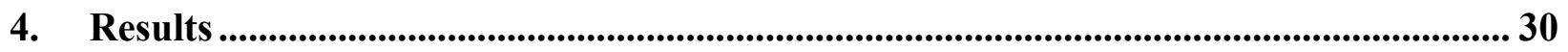

4.1 Characteristics of Ex vivo Post-Hysterectomy Cervical Tissue .......................................... 30

4.1.1 Compressive Strength of Cervical Tissue ..........................................................................

4.1.2 Fatigue Characteristics of Cervical Tissue.................................................................................34

4.1.3 Topological Features of Cervical Tissue............................................................................35

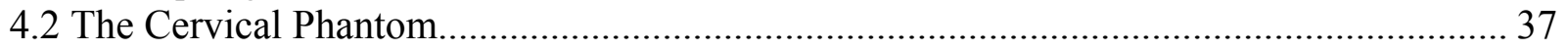

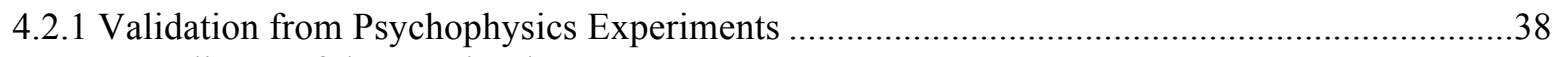

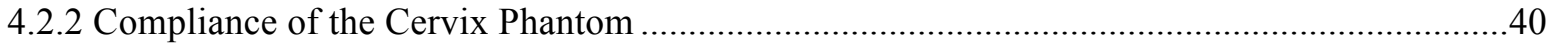

4.3 The Cervical Phantom Response to Labour Induction ...................................................... 41

4.3.1 Passive Effacement, Descent and Deflection.......................................................................

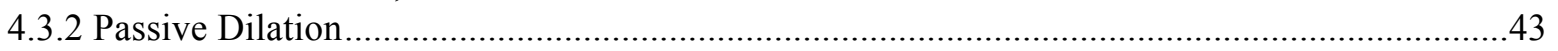




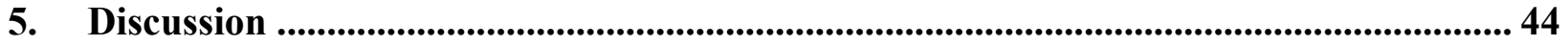

5.1 Mechanical Properties of Ex Vivo Post-Hysterectomy Tissue............................................ 44

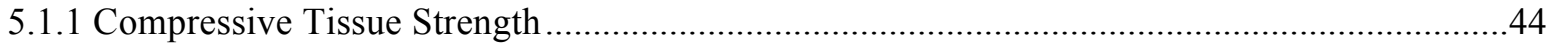

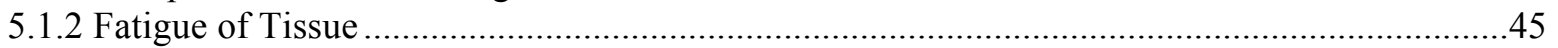

5.2 The Cervix Phantom Prototype.......................................................................................... 46

5.2.1 Comparison with Ex Vivo Post-Hysterectomy Tissue and Existing Models ……………….......47

5.2.2 Simulating Latent Labour ...............................................................................................4

5.2.3 Cervix Phantom in Simulation Training ………………......................................................49

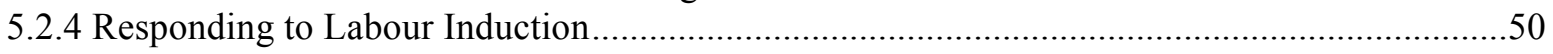

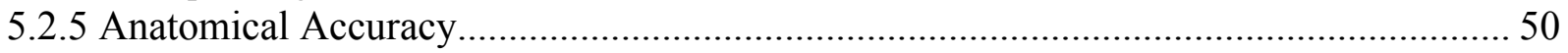

5.3 Significance of Results ............................................................................................ 51

6. Conclusions .............................................................................................................................. 52

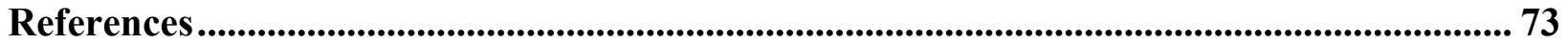

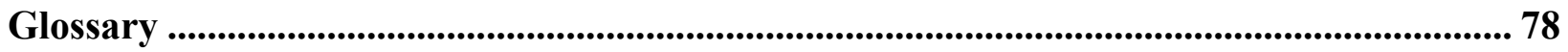




\section{List of Tables}

Table 1: The Average Thresholds Identified for Each Participant................................... 38

Table 2: Summary of Volunteer Responses to Varying Vacuum Pressures ....................... 39

Table 3: Relationship between Stress and Strain of the Cervix Phantom at Various

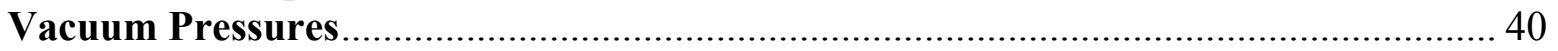

Table 4: The Vacuum Pressure Corresponding to Different Stages of Latent Labour ....... 49

Table 5: Comparison between Cervix Tissue and Phantom Prototype ............................. 51 


\section{List of Figures}

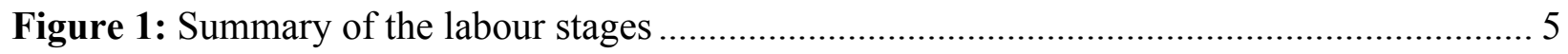

Figure 2: Dilation of the cervix is characterized by the opening of the cervical os ...................... 6

Figure 3: Effacement of the cervix, adapted from Myers et al., 2015 [18] ................................... 6

Figure 4: A sample partograph used to graphically record the progression of labour. From

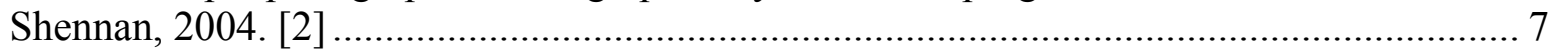

Figure 5: The Fidelis (top) and Simone (bottom) commercial birthing simulator systems, from

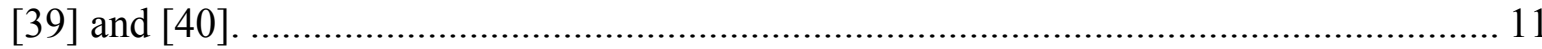

Figure 6: Inter-granular distance decreases as vacuum pressure is applied in accordance with

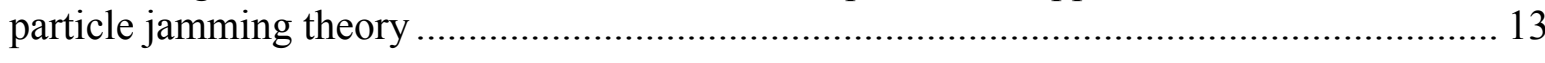

Figure 7: Fabrication method using a two-piece mold and silicone rubber cast ......................... 15

Figure 8: Flowchart describing the process to obtain post-hysterectomy tissue samples ........... 17

Figure 9: The tissue testing platform (right) and simplified representation (left) ....................... 18

Figure 10: Custom 3D-printed ABS tip (left) used to indent the cervical tissue (right).............. 19

Figure 11: The cross-sectional view showing the cervical tissue secured with the tensioned bamboo sticks (left) and elevated view of the specimen placed inside the modified Rubbermaid TakeAlongs container (right) .....

Figure 12: The experimental setup of the NextEngine 3D Scanner being used to characterize the topological features of the tissue specimen .................................................................. 20

Figure 13: Post-hysterectomy ex vivo uterus and cervical tissue sample tested at McMaster Health Sciences Centre (Hamilton, ON) ...................................................................... 21

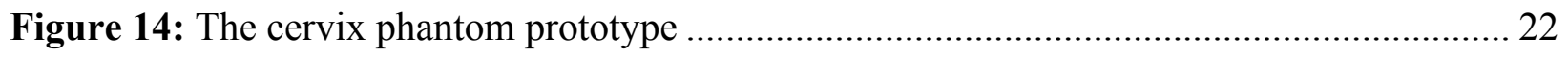

Figure 15: The testing platform was adapted to test the compliance of the cervix phantom

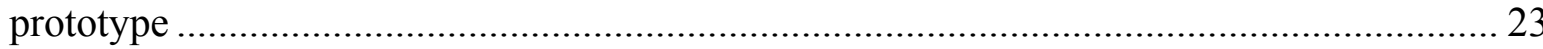

Figure 16: Participant interacting with the cervix phantom prototype in psychophysics

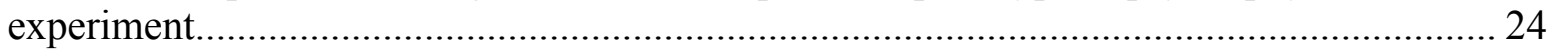

Figure 17: The use of a Foley catheter to induce labour. From Smith, 2013 [61]...................... 25

Figure 18: Representations of the cervix phantom prototype: (a) the actual cervix prototype, (b) the simplified model and (c) elevated and (d) cross-sectional views. ................................. 26

Figure 19: The cross-sectional view of the cervix balloon used to measure the effacement, deflection and descent .................................................................................................. 28

Figure 20: The elevated camera view (left) and "roipoly" function output (right) of the cervix balloon used to measure the dilation............................................................................... 29

Figure 21: Stress-strain curves of six post-hysterectomy cervical tissue samples tested at a stress point distal to the cervical os ......................................................................................... 31 
Figure 22: Stress-strain curves of ten post-hysterectomy cervical tissue samples tested at a central stress point

Figure 23: Stress-strain curves of eight post-hysterectomy cervical tissue samples tested at a stress point proximal to the cervical os

Figure 24: Comparison of cervical tissue compressive indentations conducted at different locations along the axial plane of the cervical tissue sample.

Figure 25: The effect of 10 consecutive trials and strains conducted at a central stress point on cervical tissue strength, lower strains were not affected by continuous loading in comparison to higher strains. 34

Figure 26: 3D scans contrasting post-hysterectomy sample 6 and 10 36

Figure 27: (a) Anterior and (b) Lateral views of a 3D Scanned post-hysterectomy uterus and cervix were obtained and used to produce a (c) watertight processed 3D image and (d) ABS print of the specimen.

Figure 28: The firmness of the cervix balloon model, as determined by volunteers. At 83.3 $\mathrm{mmHg}$ (vacuum), the balloon was perceived to transition from hard to soft

Figure 29: Compressive strength characteristics of the cervix phantom is similar be to the post-

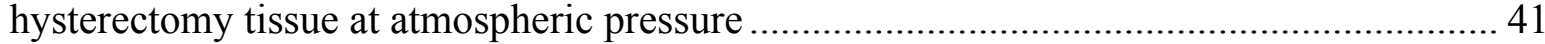

Figure 30: The response of the cervix phantom to labour induction mechanisms ................... 42

Figure 31: The amount of dilation of the cervix balloon as a function of vacuum pressure applied.....

Figure 32: Maximum principle stress of a theoretical torus is highest in the inner region and smallest in the outer region, from [63]....

Figure 33: Force relaxation response of non-pregnant (NP) and pregnant (PG) human cervical tissues shown with dotted and circled points, respectively. Two solid lines show the inverse finite element analysis (IFEA) curves for both cases. From [58].

Figure 34: The compressive strength of the cervix phantom at its softest setting $(0 \mathrm{kPa})$ is comparable to the strength of actual cervical tissue, while the softest setting of Kim's model $(20 \mathrm{kPa})$ was much stiffer, Kim's data adapted from [43]. 48

Figure 35: Actuators can be added to the outer walls of the cervix model 50 


\section{List of Appendices}

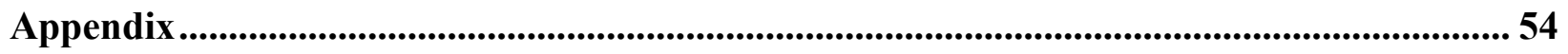

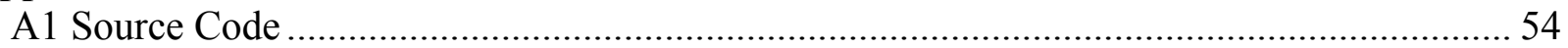

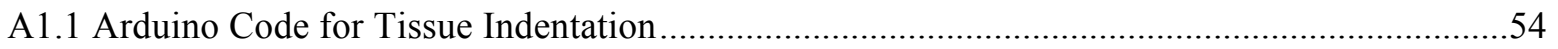

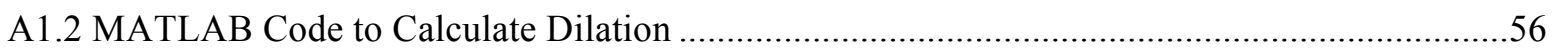

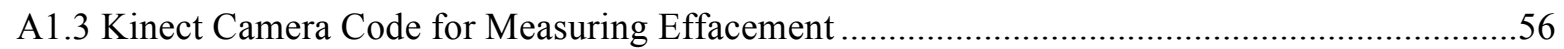

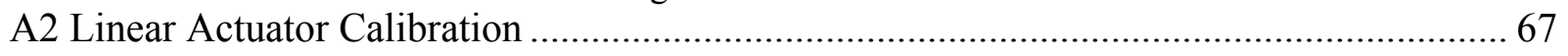

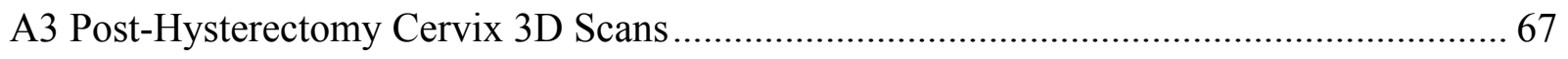




\section{Introduction}

In midwifery and obstetrics, routine deliveries can quickly become life-threatening emergencies. According to a study conducted by Guendelman et al., complications occur in as many as 1 in 5 deliveries [1]. The outcome of these labour complications depend heavily on the ability of the medical personnel to make the right decision and administer proper surgical procedure in a timely manner. For example, a prolonged latent phase of labour may require administration of oxytocin or performance of a Cesarean section [2], [3]. Similarly, accurate diagnosis of normal labour progression is critical for the prevention of unwarranted medical interventions that may increase risk for both the mother and fetus [3], [4]. Clinically, labour progression is diagnosed through observing change in cervical state during consecutive digital vaginal examinations, where the frequency of uterine contractions, dilatation of the cervix and descent of the fetal head are used as key indicators of labour progression [2]. However, while digital vaginal examinations are considered standard practice, low agreement in diagnosis between between clinicians has been observed [5]. In light of this, we propose a novel cervical model for obstetrics and midwifery simulation training to improve diagnostic accuracy.

As a student transitions from the classroom to the operating room, their lack of experience may cause errors that would result in harm to the patient, as well as a negative impact on the student's education [6], [7]. In order to minimize this risk, simulation training systems have been developed such that students can make errors in a safe environment without risking the well-being of a patient [8], [9]. Moreover, simulation systems allow for integration of knowledge and skill at a earlier stage of training than was previously possible [6], [10]. Such training methods provides an optimal method for medical students to foster good team work and communication skills critical to patient safety. Currently, there are many obstetric simulators available on the market. However, obstetric simulators can be refined in various ways. A major limitation of existing human birth simulators is the absence of a cervix with dynamic capability that can simulate the varying compliance of cervical ripening during the earlier stages of labour. Specifically, simulation of latent labour is critical, as clinical decisions such as labour induction and the need for Caesarean section occur during this stage [2]. Consequently, existing simulators are limited to simulation of the later stages of labour assuming a fully dilated cervix or require several static cervices to be manually interchanged during simulation. 
In this thesis, the design and characterization of a realistic cervix model for integration into existing human birth simulation training systems is presented. The following major tasks have been defined to accomplish this:

1. Anatomical and mechanical studies of post-hysterectomy ex vivo human cervical tissue.

2. Incorporation of findings into the design of a cervix prototype using particle jamming technology.

Anatomical features of ex vivo human cervical tissue samples will be characterized using the NextEngine 3D Scanner [11]. Mechanical properties will be determined using a mechanical indentation device along the plane perpendicular to the neck of the cervix. Indentations will be made at three locations evenly spaced from the external cervical os, to the edge of the cervix. While the mechanical strength of the ex vivo cervical tissue specimens will be stiffer than that during pregnancy, values will provide a starting point for our model to simulate at the onset of labour. Future studies can utilize the same testing platform to study pregnant samples, when they become available. Findings will be incorporated into the design of a cervix prototype. This prototype will be capable of softening through adaptation of particle jamming technology. Specifically, a non-porous, flexible toroid-shaped latex balloon loosely packed with granular coffee grounds will be controlled with a pneumatic pump to vary the vacuum pressure. In accordance with particle jamming technology, the coffee grains will flow at atmospheric pressure, when excess interstitial fluid (i.e. air) is present, but form a compact, hard solid when a vacuum is applied to removed the fluid [12]. The mechanical indentation device will be used to measure compliance of the model in response to varying vacuum pressures. Additionally, tactile psychophysics experiments will be conducted for the cervix phantom prototype to determine the vacuum pressure ranges that corresponded to the human perception of 'hard' and 'soft'. Finally, the system will extend to measure passive dilation and effacement of the model in response to labour induction will be measured using Kinect cameras.

Work presented in this paper will serve as foundational for the development of a realistic cervical model for simulation training. The phantom prototype presented will be the first of its kind to offer a dynamic cervix focused on simulation of latent labour, and promises significant improvement over didactic teaching methods in teaching nurses, midwives and medical residents in labour and delivery. However, the cervix model utilizes commercially available toroid-shaped balloons that lack anatomical accuracy. In order for the model to be a faithful representation of the 
anatomical cervix, the proposed design will need to be retrofitted to a custom-shaped flexible balloon. 3D printing technology is an inexpensive and practical manufacturing option to be explored to that end. 


\section{Survey of Literature}

A thorough survey of pertinent literature is presented in the following section. To start off, the dynamics of labour from an anatomical and physiological perspective was studied. Clinical tools to diagnose the progression of labour were also determined. Following this, the efficacy of simulation training for healthcare professionals, including obstetricians and midwives, was reviewed. Both commercial and experimental obstetric simulation systems are presented. Finally, the technological advances of soft robotics and 3D printing as it relates to human birth simulation systems are summarized.

\subsection{Labour}

\subsubsection{Stages of Labour \& the Role of the Cervix}

Labour and delivery is well understood process that can be characterized by three clear stages, as shown in Figure 1 [2]. In the first stage of labour, the cervix undergoes major biochemical changes and is pulled open passively by uterine contractions to $10 \mathrm{~cm}$ dilation [13]. The second stage is characterized by the delivery of the baby following full dilation. The third and final stage occurs from the delivery of the baby to the delivery of the placenta. Following labour, the uterine walls undergo continuous, coordinated contractions to compress local blood vessels and form a retroplacental clot to control bleeding at the placental site [14].

The first stage of labour can be further divided into three phases, namely: (1) latent labour, (2) active or accelerated labour and (3) transitional labour. Each of these phases can be characterized by the frequency and intensity of uterine contractions, dilation of the cervix and descent of the baby's head [2]. At the onset of labour, the cervix is a firm cylindrical structure, 2 $\mathrm{cm}$ in length and 1-2 $\mathrm{cm}$ wide, that forms the distal component of the uterus [15]. During latent labour, the cervix undergoes remodeling of the extracellular matrix components and reorganization of the collagen fibrillar network [13]. This process, termed cervical ripening, involves a decrease in collagen concentration due to a decline in collagen synthesis and increased collagenase activity in the originally fibrous cervix tissue [15]. Cervical ripening is also associated with an increase in prostaglandin, estrogen and cytokine levels [13]. As the cervix softens, it begins to dilate and efface in response to uterine contractions. Dilation, as shown in Figure 2, is characterized by the opening 
of the cervical os, and is measured in centimeters [16]. Effacement, as shown in Figure 3, is the corresponding thinning of the cervix from $2 \mathrm{~cm}$ to becoming paper thin, and is reported as a percentage [17]. Latent labour progresses up to $3 \mathrm{~cm}$ of cervical dilatation, $80 \%$ effacement and can last up to 8 hours without required medical intervention [2], [3]. The active phase of labour is characterized by an accelerated dilation rate of $1 \mathrm{~cm} /$ hour, during which the cervix opens from 3

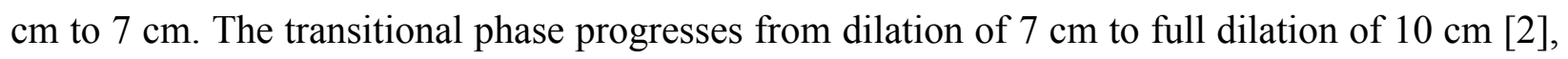
[13]. At this point, the cervix has fully opened and is ready for the newborn's head to pass through and labour progresses from the first to second stage.

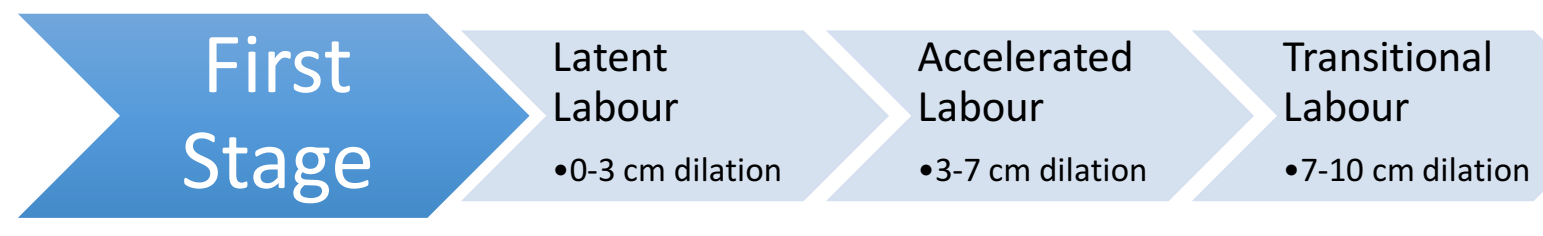

\section{Second Stage

Figure 1: Summary of the labour stages 

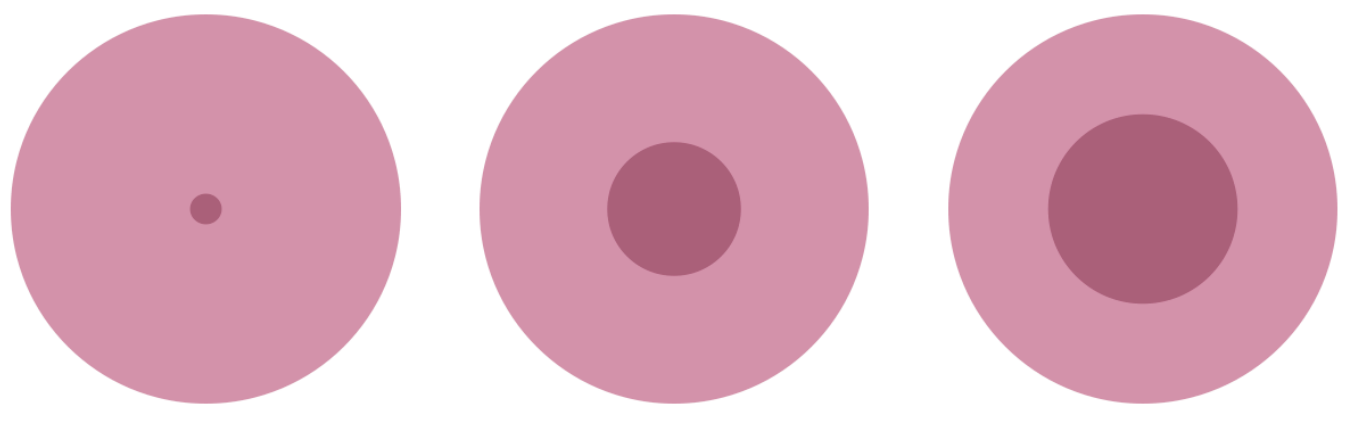

Figure 2: Dilation of the cervix is characterized by the opening of the cervical os

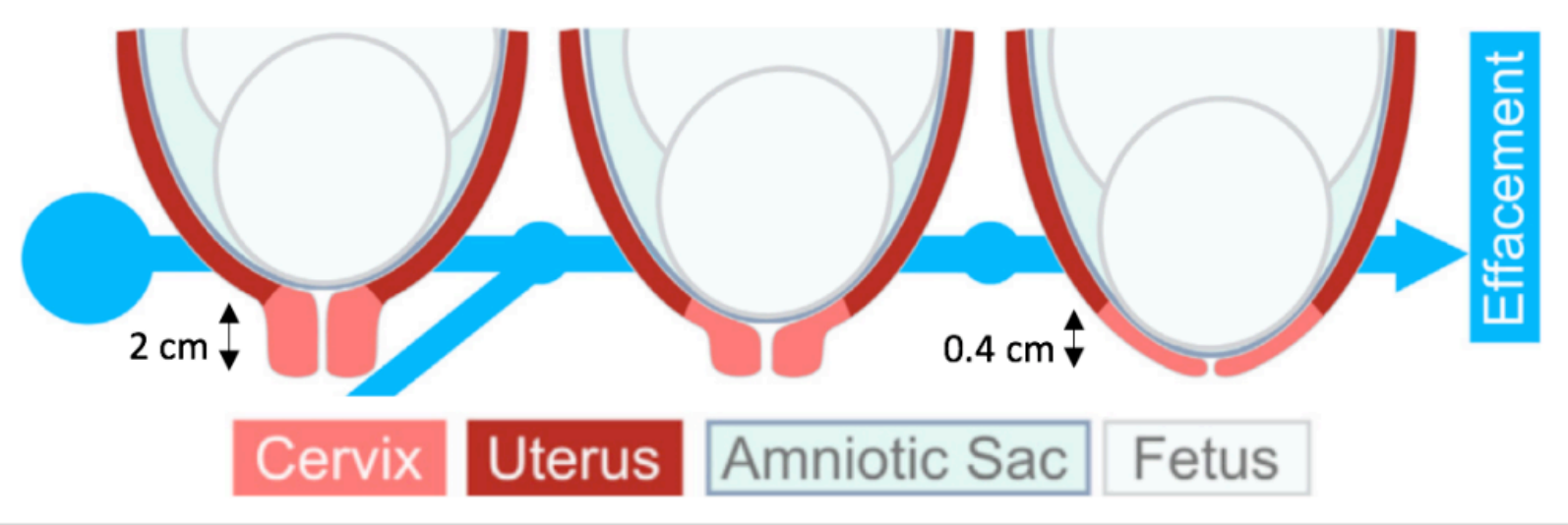

Figure 3: Effacement of the cervix, adapted from Myers et al., 2015 [18]

\subsubsection{Clinical Diagnosis of Labour Stages}

Accurate diagnosis of labour progression is critical to ensure maternal and fetal wellbeing. A prolonged latent phase of labour may indicate the need for medical intervention, such as administration of oxytocin or performance of a Caesarean section [2], [3]. Similarly, recognition of normal latent labour progression is important for the prevention of unwarranted medical interventions that may increase risk for both the mother and fetus [3], [4]. For example, proper selection of women for the labour induction is important, because induction increases the risk of postpartum and neonatal complications [19]. Labour stages are identified and diagnosed through 
observing change in cervical state during consecutive digital vaginal examinations [2]. During these examinations, the frequency of uterine contractions, dilation of the cervix and descent of the fetal head are used as key indicators of labour progression.

In clinical practice, the progress of labour is graphically recorded using a partograph and quantified in a Bishop score [2], [20]. In a partograph, as shown in Figure 4, cervical dilation and descent of the fetal head is plotted against time. Strength and frequency of contractions, state of liquor, and position moulding are also monitored and recorded on the partograph. Similarly, the cervix-focused Bishop score is a scoring system used to monitor the stages of labour based on five components observed during vaginal examination: cervical dilation, cervical effacement, cervical consistency (softening), cervical positioning and fetal station [20]. Each of the five categories is scored out of 3 , for a maximum of 15 points. The Bishop score has been documented as an effective method to predict the need for medical intervention [21]. A Bishop score greater than 8 indicates that the woman will undergo successful vaginal birth without the need for medical intervention. In contrast, a Bishop score of 5 or less suggests the need for labour induction.
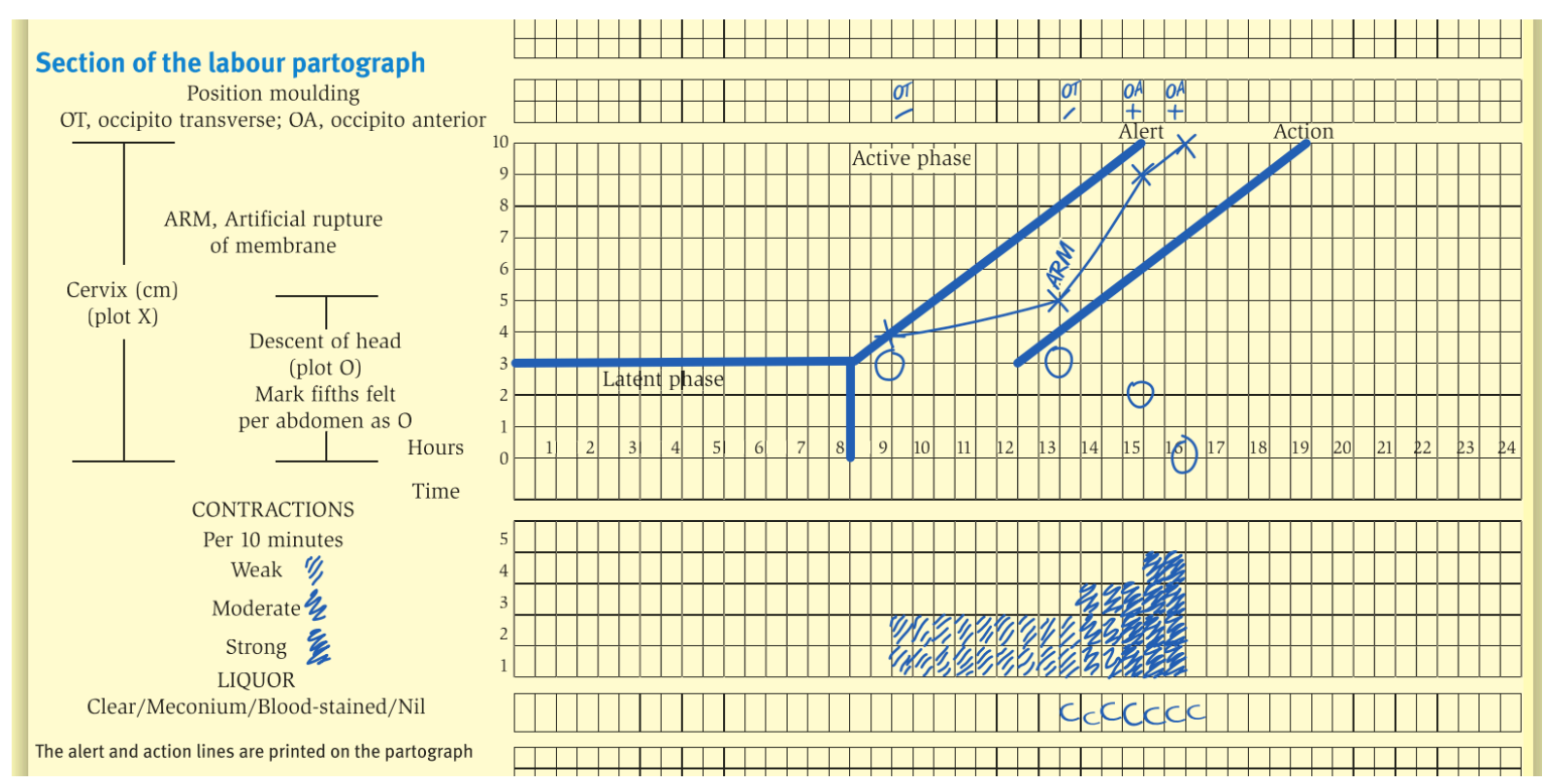

Figure 4: A sample partograph used to graphically record the progression of labour. From Shennan, 2004. [2]

While digital vaginal examinations are considered standard practice, low reproducibility in Bishop scoring has been observed [5]. Garcia-Simon et al. conducted a study to evaluate the reliability of the Bishop score when performed by first year obstetrics residents [4]. Inconsistencies 
in the inter-observer agreement of Bishop scorings were observed, with a coefficient of variation ranging between 8.56 to $29.31 \%$ [4]. Similarly, Faltin-Traub et al. studied the inter-observer agreement in Bishop scoring between three pairs of experienced obstetricians, and observed

varying inter-class correlations ranging from 29 to 70\% [22]. Although observed differences are still within acceptable clinical range, efforts to mitigate observer differences are needed to improve health outcomes of mothers and newborns. Transvaginal ultrasound examination has been proposed as an alternative, due to its high reproducibility and inter-observer reliability [4], [22]. However, ultrasound examinations are more costly, and not available in low-resource settings. Therefore, improving the accuracy of digital examination accuracy through the introduction of better simulation training models is preferred.

\subsubsection{Induction of Labour}

When medical complications occur in pregnancy, labour inductions are performed to minimize risks of pregnancy continuation by initiating vaginal birth within 48 hours [19]. Both pharmacological and mechanical methods are clinically used to achieve labour induction. Pharmacological agents include locally applied prostaglandins [23] and oxytocin augmentation [23], while mechanical methods involve the use of catheter balloons [19], [23], [24] and membrane sweeping [25], [26].

Prostaglandin $\mathrm{E}_{2}\left(\mathrm{PEG}_{2}\right)$ and oxytocin administration are commonly used pharmacological induction methods. Prostaglandins are a class of lipid compounds with hormone-like activity that signals cervical ripening, fetal membrane rupture and uterine contractility [27]. When cervical ripening does not progress naturally, synthetic $\mathrm{PEG}_{2}$ is applied intra-vaginally or intra-cervically to stimulate the softening of the cervix and induce labour [27]. For women with a low Bishop score (e.g. 5 or less), an agent such as prostaglandin can administered to induce labour contractions [28]. While pharmacological agents are effective, side-effects, including uterine hyper-stimulation and fetal distress, have been reported [23], [24]. Therefore, mechanical induction methods have been recommended as a potentially safer and more economical alternative.

Common mechanical induction methods include the use of the Foley catheter and membrane sweeping by clinicians. Membrane sweeping is a mechanical induction technique that stimulates the production local prostaglandins [25]. Membrane sweeping is performed by a clinician during a vaginal examination, where the clinician's finger detaches the membranes from 
the lower uterine segment by circular movements. In contrast, the Foley catheter is a device inserted through the cervical canal into extra-amniotic space, where it is inflated or infused with saline solution [29]. Once applied, the mechanical action of the catheter strips the fetal membranes of the lower uterine segment and stimulates local prostaglandin production to improve consistency and effacement of the cervix [29]. The use of the Foley catheter has been reported to shorten induction to delivery interval, decreasing the rate of Caesarean sections and increasing the rate of spontaneous vaginal delivery [30]. Moreover, studies show that Foley catheter use causes less fetal distress, is safe for out-patient use and is recommended for resource-limited settings due to its lower cost [23], [24].

\subsection{Simulation Training Systems}

\subsubsection{Efficacy of Simulation Training}

The effective use of simulation systems has been reported in both obstetric and midwifery curricula [9], [31], [32]. However, the specific demands of simulation systems differ for these two different medical groups. For example, Health Workforce Australia (HWA) reports that the use of simulation training in midwifery programs focuses on providing preparation in emergency situations, training for teamwork and improvement of communication skills [32], [33]. In contrast, obstetricians tend to use simulation systems to practice instrumented deliveries during complicated births, such as vaginal breeches and shoulder dystocia, that require the use of forceps or vacuum [34]. Due to the relative infrequency of these complications in the clinical setting, there was traditionally a lack of hands-on practical training of obstetric maneuvers that ultimately led to an increased risk for a variety of birth trauma [35].

The positive effects of simulation training systems have been well-documented in various studies [6], [9], [36]. Robertson reported that students expressed that the use of simulation training had increased their knowledge, preparing them for clinical practice [9]. Jude et al. demonstrated that third-year medical students who practiced on obstetric simulators were more confident in performing most portions of vaginal delivery with minimal supervision [6]. Finally, Holstrom et al., found that in addition to increased confidence, medical clerkship students that were trained with simulation systems demonstrated higher oral and written examination scores than students that were given formal structured instruction [36]. 
Benefits of simulation training can also be assessed with respect to the improvement of cognitive ability. Particularly, simulation training improves upon the attentional capacity of an individual. In cognitive psychology terms, attention refers to the ability for one to focus mentally on a task [7]. The ability for humans to focus is limited by our attentional capacity, and as such one can only focus on a finite amount of information or stimuli at a given instance. When an apprenticeship student is presented with tasks that require attentional resources that exceed their threshold of attentional capacity, they are no longer able to perform effectively. Through the use of simulation training, a novice surgeon will acquire automated psychomotor skills and spatial judgments required during delivery such that fewer attentional resources are occupied. This allows for the simulation-trained novice to concentrate on learning how to handle unexpected complications and refine their surgical skills, minimizing the risk to patients in operating room.

\subsubsection{Commercial Simulation Training Systems}

Medical simulators are currently commercially available in a wide spectrum, including verbal role playing and actor patients, part-task trainers (PTTs), computer screen-based programs, haptic trainers, virtual reality trainers and high fidelity electronic manikins [37]. These simulators range in portability and cost. Generally, PTTs and screen-based are low in cost and very portable, while virtual reality, haptic trainers and electronic manikins are more costly and less portable. Different simulators can also be used in combination with one another. Such simulators, known as hybrid simulators, may use an obstetrical PTT in combination with actor for realistic simulation of patient [37]. Such approaches accomplish augmented reality at a lower-cost than high fidelity models.

Some commonly used obstetric simulators include the Practical Obstetrical MultiProfessional Training (PROMPT) birthing simulator by Limbs \& Things [38], the Noelle Simulator by Gaumard Scientific [39], the Simone Childbirth Birthing Simulator by American 3B Scientific [40], and the Fidelis Maternal Fetal Simulator by CAE Healthcare [41]. PROMPT is a PTT that consists of an abdomen and pelvis that can be used to simulate shoulder dystocia, breech delivery or normal vaginal delivery [38]. The Noelle simulator, as shown in Figure 5 (top), is a high fidelity electronic manikin equipped with an automatic birthing system, interactive computer screen and resuscitation baby with an intubatable airway and umbilical vein [9], [39]. Simone, as shown in Figure 5 (bottom), is a PTT/haptic simulator consisting of a pelvis and video monitor for 
simulation of instrumented delivery skills, such as vacuum extraction and forceps placement [40]. Finally, the Fidelis Simulator is a realistic medical robot with dilating pupils and palpable soft skin [41]. The Fidelis is capable of simulating uterine contractions and leg and hip articulations for practice of childbirth positioning and maneuvers [41]. Post-simulation assessment can be conducted using records of birth positioning and treatments detected and stored by the simulator [41].

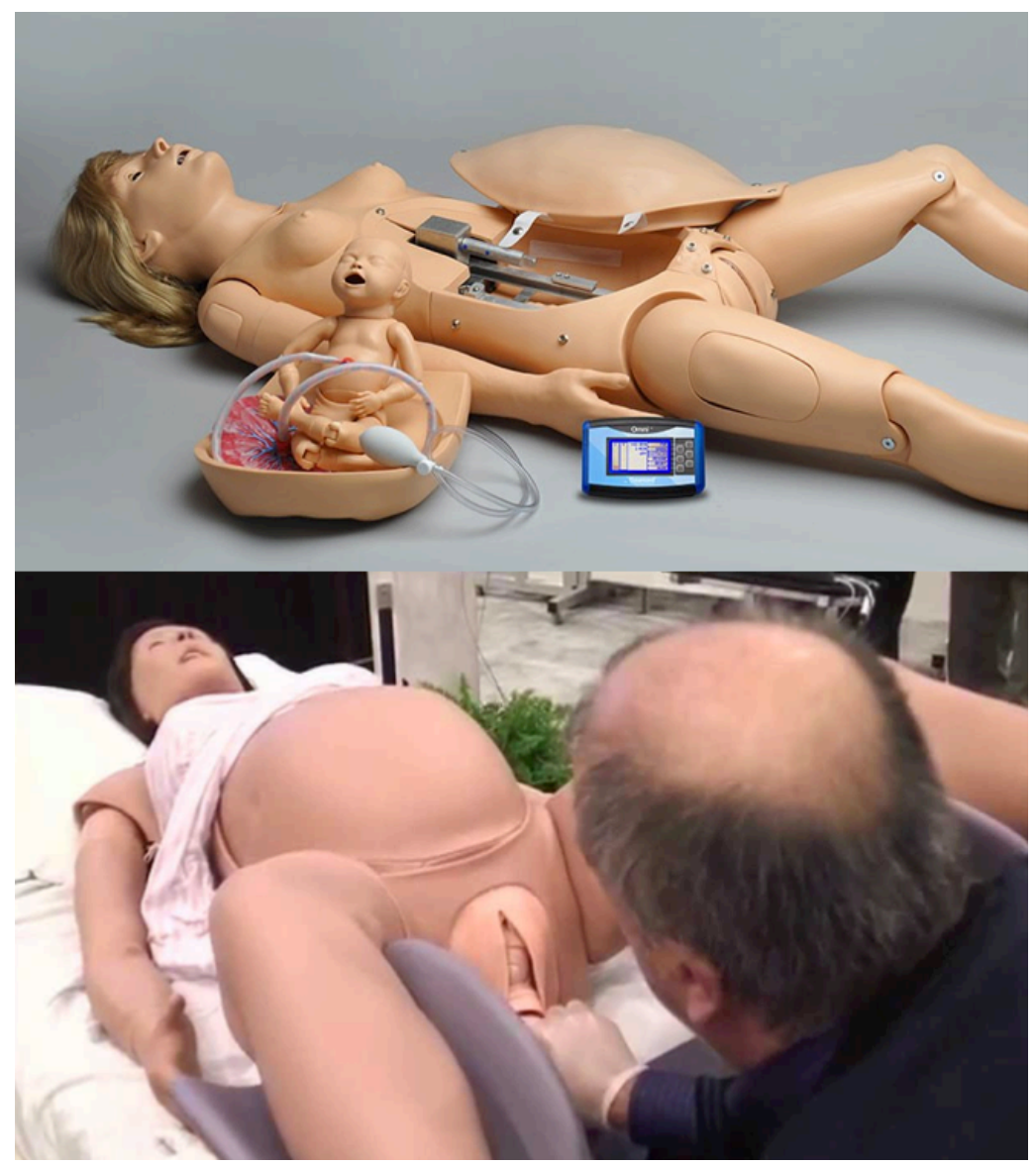

Figure 5: The Fidelis (top) and Simone (bottom) commercial birthing simulator systems, from [39] and [40].

\subsubsection{Experimental Cervical Models}

Research efforts continue to be undertaken to improve the realism of models such that a plenary birthing experience can be simulated. Kim et al. designed a novel birthing simulator consisting of a maternal model and instrumented fetal model [34]. The design of this simulator focused on performing complicated births, providing opportunities for clinicians to practice these scenarios 
more frequently than they occur clinically. The simulator also measures clinician-applied forces using a piezo-resistive pressure sensor embedded in clinician glove and a wireless electromyograph (EMG) device. While effective for simulation of complicated delivery processes, the birthing simulator designed by Kim et al. is limited to second and third stages of labour, as it as assumes a fully dilated cervical opening. To that effect, York et al. designed a second-trimester uterine model for use in second trimester dilation and evacuation (D\&E) simulation training [42]. Six interchangeable cervices were created with different degrees of dilation and textures: cervix 1 had a dilation of $2 \mathrm{~cm}$ and a wall thickness of $2.5 \mathrm{~cm}$, cervix 2 had a dilation of $3 \mathrm{~cm}$ and a wall thickness of $2 \mathrm{~cm}$, cervix 3 had a dilation of $4 \mathrm{~cm}$ and a wall thickness of $1.5 \mathrm{~cm}$. The remaining three cervices had the same dimensions, but were fabricated using commercially available Ecoflex Supersoft Silicone Rubber, Shore 30 and Shore 10. The uterine model was effective in simulating the compliance of the cervix. However, it required manual changing between the different cervices in order to simulate dilation and softening. Hence, it was ineffective in simulating the progression of latent labour, which would require a singular dynamic cervix model.

A dynamically compliant cervical model was designed and characterized by Jung Kim in his MASc thesis [43]. In this model, a 3D printed thermoplastic elastomer was filled with granular permitted and connected to a pneumatic system such that the compliance could be regulated. The cervix phantom was $3.0 \mathrm{~cm}$ in diameter, and had a spring constant that ranged from $5.3 \mathrm{~N} / \mathrm{m}$ to $10.5 \mathrm{~N} / \mathrm{m}$ as the pressure inside the phantom was varied. The cervical model was anatomically accurate and dynamically compliant. However, it was found to be two orders of magnitude stiffer than actual cervical tissue [43]. In order to address this issue, latex or silicone rubber models should be explored.

\subsection{Engineering Technologies}

\subsubsection{Soft Robotics and Particle Jamming}

Soft robotics is an emerging field that addresses the need for robots to interact safely with humans and natural environments in medical and human assistance applications. Soft robots need to have compliant characteristics such that they can be deformed to adapt various shapes and motions [44]. Unlike rigid-structured robots, which are only programmed to withstand a limited number of forces and events, soft robots can respond passively to externally exerted forces within a reasonable range 
[45]. Studies have shown the efficacy of using various materials, such as electro-active polymers [46], elastomers [47] and hydrogels [48], in the design of soft robots. However, much work still needs to be done to preserve compliance while ensuring mechanical structure and strength are not compromised.

Particle jamming is a mechanism that has been proposed to acquire dynamically compliant soft robots with high strength characteristics. With this mechanism, a granular media contained inside a non-porous flexible membrane can be manipulated to exhibit both fluid and solid-like states using vacuum pressure [49]. As shown in Figure 6, the distance between individual grains of media decreases when a vacuum is applied to the non-porous membrane. When high vacuum pressures are exerted on these jammed systems, they exhibit high strength-to-weight ratio. Conversely, when low vacuum pressures are applied, these systems become flexible in shape. Numerous studies have shown the efficacy of particle jamming in soft robotics [12], [49], [50]. Amend et al. designed a passive gripper that utilized positive and negative jamming pressures to release and grip various objects [50]. Cheng et al. designed a manipulator with jammed and unjammed segments to achieve complex and highly articulated shapes and high strength-to-weight performance [49]. Follmer et al. demonstrated the use of particle jamming to achieve Organic User Interfaces (OUIs). Four prototypes were developed, including a malleable tabletop for 3D modelling, a haptic information channel, a haptic feedback tablet and a shape changing mobile phone that could be moulded to any desired shape in its unjammed state.

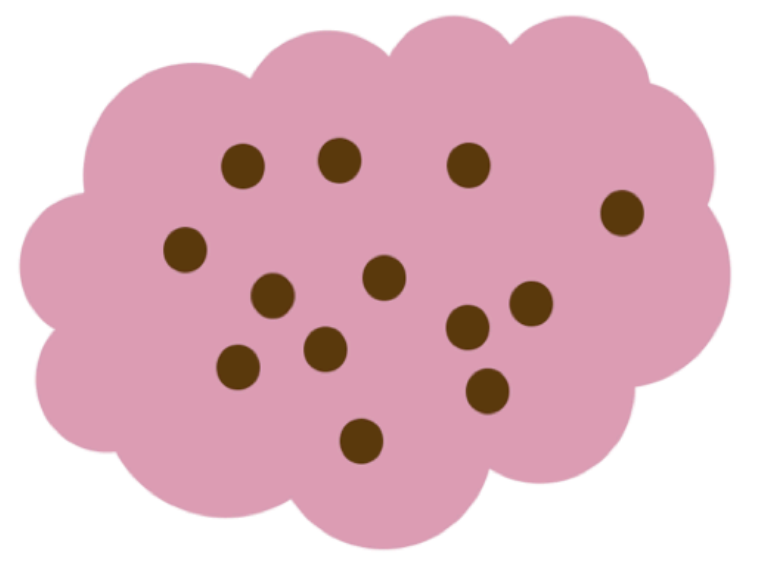

Apply vacuum pressure

Figure 6: Inter-granular distance decreases as vacuum pressure is applied in accordance with particle jamming theory 
Several lightweight granular materials have been explored for use in particle jamming, including coffee grounds, sawdust, diatomaceous earth and hollow glass spheres (10-50 microns diameter) [12], [49], [51]. Compression tests conducted by Cheng et al. revealed that coffee grounds exhibited the highest strength-to-weight ratio in comparison to other common granular materials [49]. Stress-strain curves for coffee grounds yielded a typical material response, including an effective elastic region followed by a plateau region. Furthermore, qualitative tests revealed that coffee grounds were easier to unjam and exhibited greater flow in comparison to other materials. The strong performance of coffee grounds is expected to be due to the significant surface roughness of coffee particles which increases inter-particle friction and inherent moisture of the coffee grounds that contribute to bulk strength [49].

\subsubsection{D Printing Technology}

3D printing is a revolutionizing technology that is being adopted for use in many fields, including manufacturing, robotics, engineering and medicine. 3D printed objects are first designed using computer-aided design (CAD) technology. The digital files are then transferred to a 3D printer, where material is deposited in cross-sectional layers that bond together to form the object. Many different materials can currently be 3D printed, including plastic, liquid resin, gypsum powder and metals [52]. 3D printing technology is an attractive rapid prototyping tool, because it can be used to fabricate structurally complex configurations quickly and at a low-cost. Researchers have used 3D printing to design and fabricate structurally complex molds of acrylonitrile butadiene styrene (ABS) that can then be cast with polymer materials [47], [53]. Researchers have also explored the use of 3D printing in soft robotics. Bartlett et al. designed a multi-material 3D printed soft robot using the Connex500 Stratasys printer [54]. Flexible filaments have also been developed for desktop 3D printers, such as the thermoplastic Fenner Drives Ninjaflex elastomer filament [55].

Overall, there are many applications for this technology in medicine, including the potential for patient-specific implants, prostheses and realistic anatomical models education and planning [52]. For example, 3D printing can be used to design anatomically accurate human birth phantoms to be incorporated into simulation training systems. In Kim's thesis, the use of 3D printing to fabricate a to-scale cervical phantom was explored [43]. Several different fabrication methods were explored, including the design of 3D printed molds and 3D printing soft materials. 
As shown in Figure 7, a two-part negative ABS mold can be designed using CAD technology and printed in ABS using the Lulzbot Taz 4 3D printer. Using this mold, a phantom can be casted from Smooth-On silicone rubber, which has elastic properties that allow it to be stretched without tearing or distortion [47]. However, the resulting phantom demonstrated poor repeatability and inconsistent wall thickness, susceptible to tears when subjected to vacuum pressures [43].

A $0.7 \mathrm{~mm}$ thickness 3D printed Ninjaflex cervix phantom model was also presented by Kim [43]. This phantom was to-scale and capable of softening using particle jamming. However, Kim's model remained to be two orders of magnitude stiffer than actual cervical tissue [43]. Overall, Kim's comprehensive study of soft fabrication methods demonstrates that 3D printing of soft materials remains to be limited and ineffective at present. In order to achieve required softness to simulate the cervix, it is recommended that the use of a non-porous commercial latex phantom be explored.

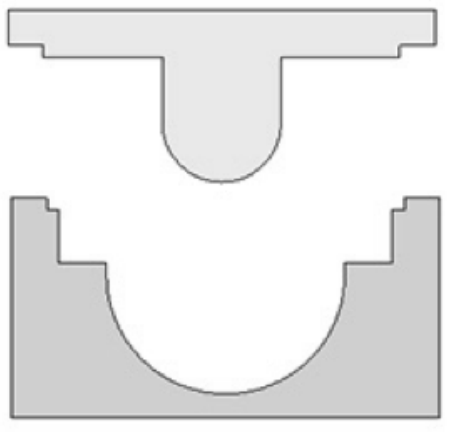

Two-piece mold

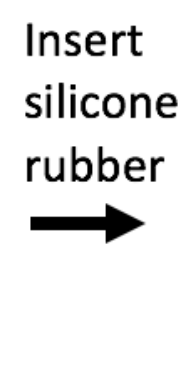

Figure 7: Fabrication method using a two-piece mold and silicone rubber cast 


\section{Methodology}

The objective of this work is to design and characterize a realistic cervix model for integration into existing human birth simulation training systems. To this end, topological and mechanical studies of post-hysterectomy ex vivo human cervical tissue were conducted. Following this, findings were incorporated into the design of a cervix phantom prototype using particle jamming technology. The ability for the cervix phantom to soften, passively dilate and efface were evaluated. The methodology is detailed in the following section.

\subsection{Ex Vivo Cervical Tissue Experimentation}

\subsubsection{Tissue Acquisition}

Tissue testing was conducted on ten ex vivo whole uterus and cervix specimens acquired from consented hysterectomy patients after surgical removal at McMaster Health Sciences Centre (Hamilton, ON). Hysterectomy patients require surgical removal due to medical conditions such as cervical or uterine cancer, development of fibroids, endometriosis or a prolapsed uterus [56]. The cervix portion of the test specimens appeared to be intact after hysterectomy, were judged to be have maintained their structural integrity and were representative of healthy tissue. Tissue was collected from patients ranging from 31-52 years of age.

Due to availability, only non-pregnant tissue samples were collected. However, using the same experimental apparatus and protocol, pregnant tissue samples could be collected from rare hysterectomy cases of patients with placenta accreta. These high-risk pregnancy cases result from abnormal growth of the placenta into the uterine wall [18]. Collective results from these different hysterectomy samples are expected to exhibit characteristic trends that give insight into mechanical properties of the cervix at the onset of labour.

Figure 8 shows the process to obtaining viable post-hysterectomy tissue samples for experimentation. The process began with receiving the list of patients scheduled for a hysterectomy surgical procedure. Following this, additional criteria had to be met to ensure that experiments could be conducted. Specifically, specimens were not used when pathologists identified hyperplasia or when the sample was morcellated during surgery. Hyperplasia is a condition characterized by gross enlargement of the specimen. Specimens exhibiting hyperplasia 
may be cancerous, and thus need to be processed by pathology immediately and unsuitable for experimentation. Morcellation is a technique used to break apart tissue samples into smaller pieces for easier removal during minimally-invasive laparoscopic surgery. Morcellated samples did not have an intact cervix and hence could not be used.

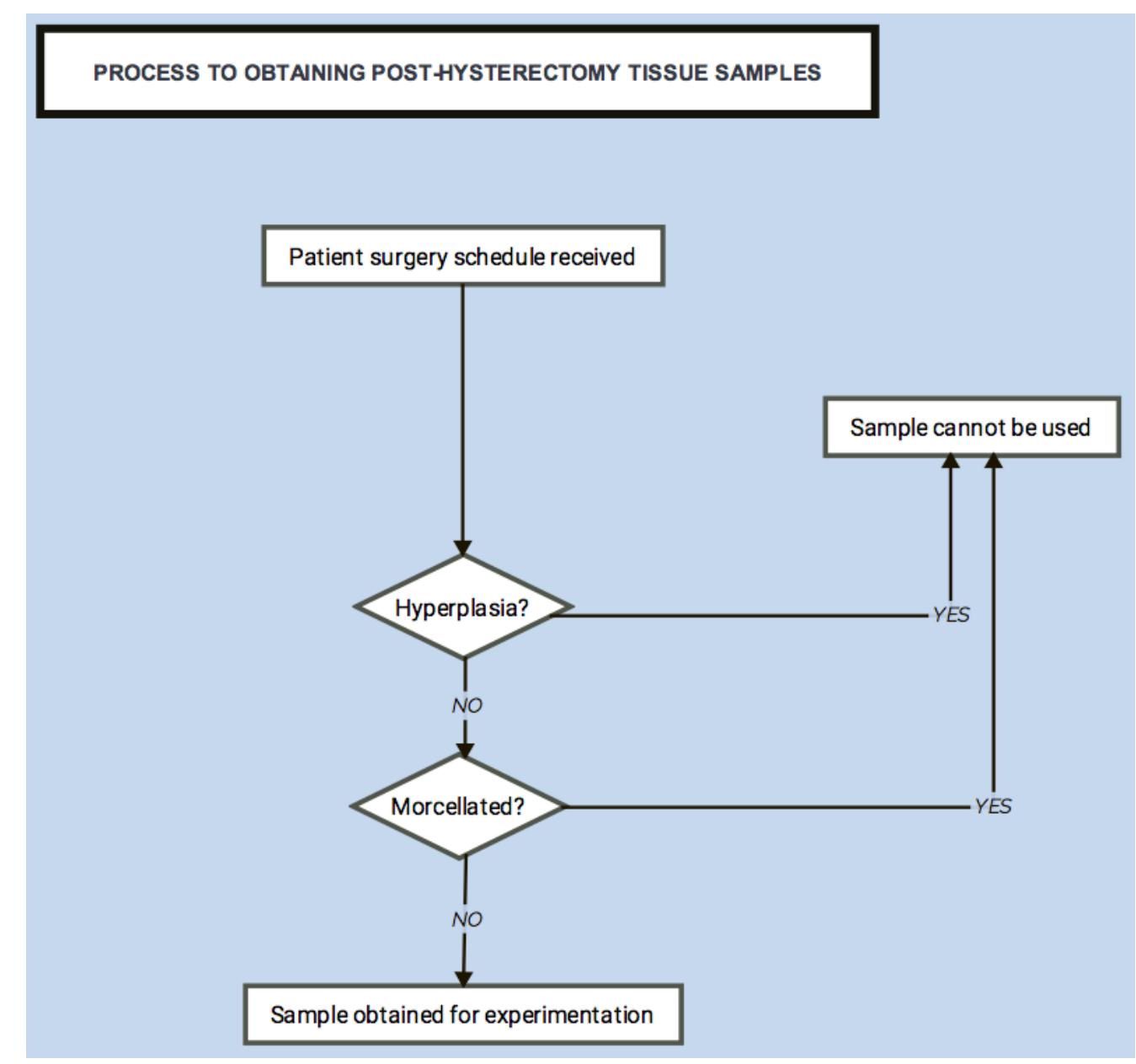

Figure 8: Flowchart describing the process to obtain post-hysterectomy tissue samples

\subsubsection{Testing Platform}

A testing platform, as shown in Figure 9, was developed to test the strength of the tissue in response to four pre-programmed indentation distances. Force corresponding to these indentations were measured using the OHAUS Scout Pro SP2001 scale, which has a capacity of 0-2000 $\mathrm{g}^{1}$ and sensitivity up to $0.1 \mathrm{~g}$. The Firgelli L12 linear actuator, with a stroke length of $5.0 \mathrm{~cm}$ and a

\footnotetext{
${ }^{1}$ Corresponding to force range of $0-19.6 \mathrm{~N}$
} 
positional accuracy of $0.002 \mathrm{~cm}$, was used to indent the tissue by precise displacements. Positional accuracy was verified through measuring programmed distances using a digital Vernier caliper. The linear actuator was housed in a custom designed 3D printed case and secured using MicroRAX frame. The linear actuator was controlled using an Arduino microcontroller and Freescale MC33926 full H-Bridge. A disposable ABS tip with a $4.5 \mathrm{~mm}$ diameter was custom designed and 3D printed to attach to the linear actuator and indent the tissue, as shown in Figure 10.

The tissue specimen was placed inside a disposable 5.2 cup Rubbermaid TakeAlongs square container with a custom circular cut-out to secure the neck of the cervix in place, as shown in Figure 11 (right). This allowed the tissue to be positioned such that the cervix portion of the specimen was perpendicular to the indentation tip. To ensure equal force distribution, two bamboo sticks tensioned with elastic bands were also used to support the neck of the cervix (Figure 11, left). All materials in direct contact with the tissue sample, including the indentation tip, container and bamboo sticks, were disposed after each experiment to prevent contamination. Tissue was returned to the McMaster Health Sciences Centre Anatomical Pathology lab personnel for regular analysis, storage and proper disposal.
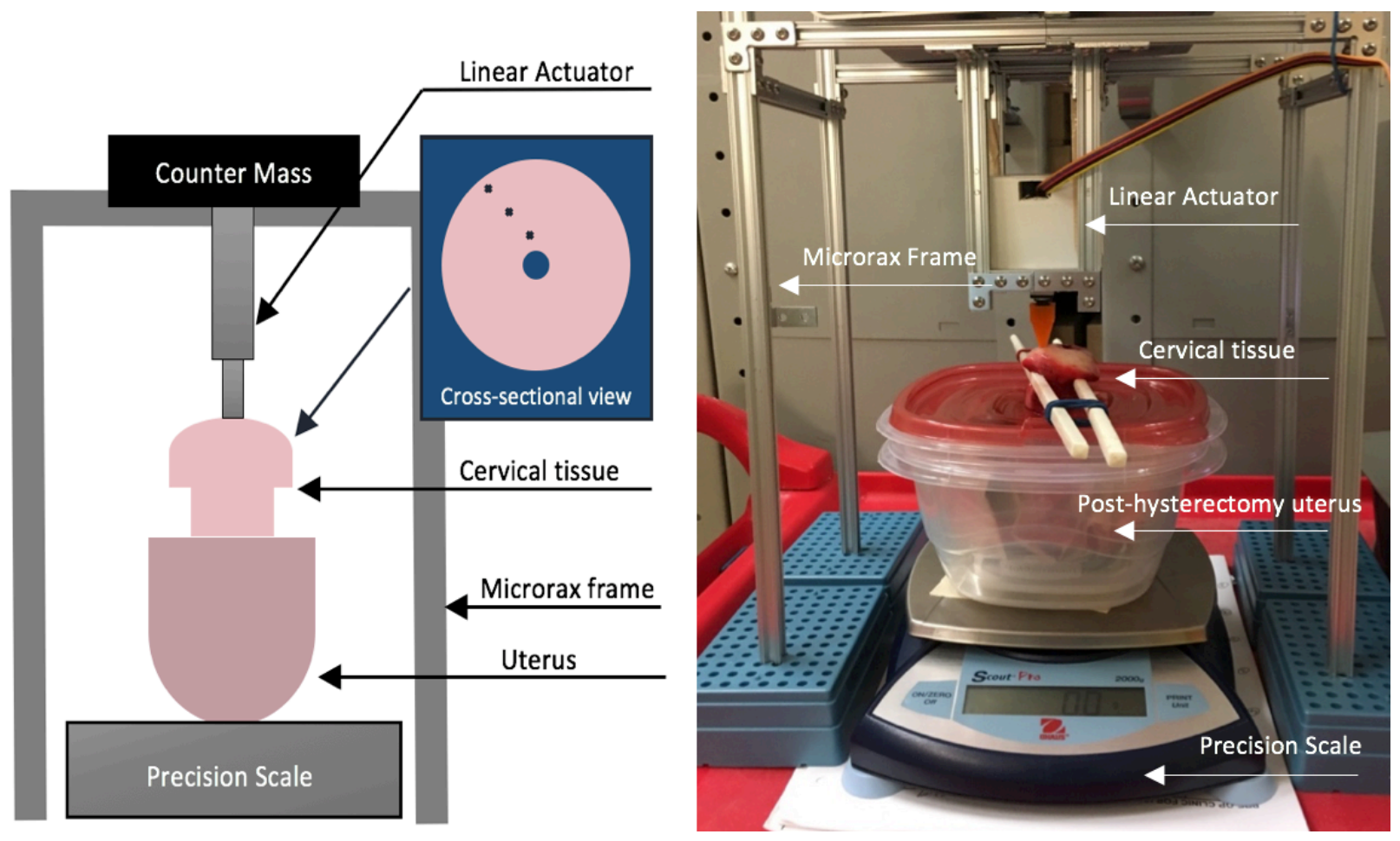

Figure 9: The tissue testing platform (right) and simplified representation (left) 


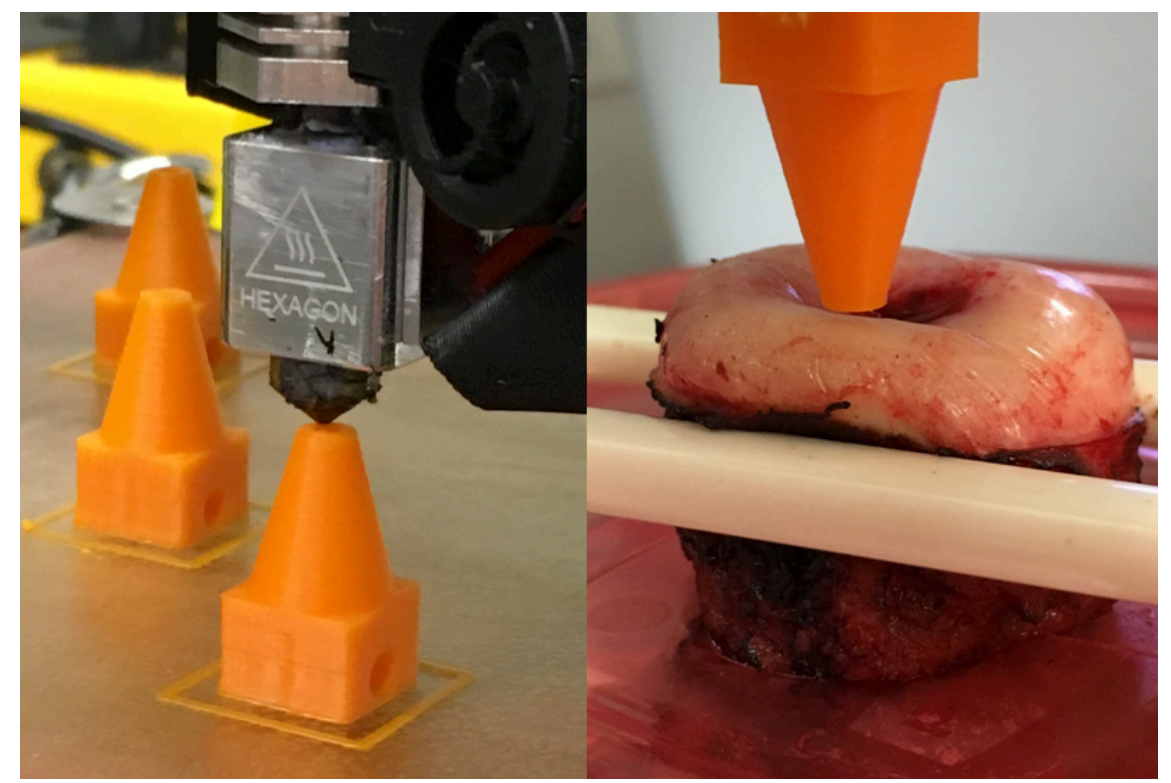

Figure 10: Custom 3D-printed ABS tip (left) used to indent the cervical tissue (right)
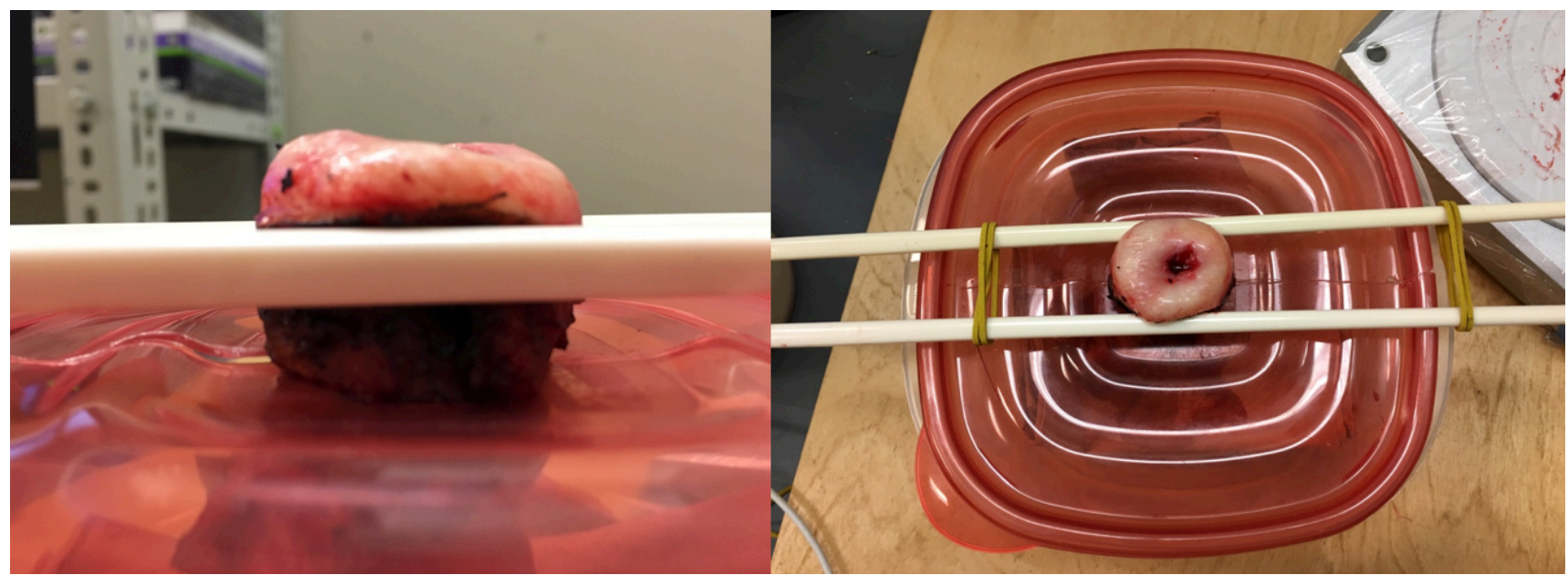

Figure 11: The cross-sectional view showing the cervical tissue secured with the tensioned bamboo sticks (left) and elevated view of the specimen placed inside the modified Rubbermaid TakeAlongs container (right)

\subsubsection{Tissue Characterization}

Due to the anisotropic nature of cervical tissue [18], tests were conducted with the whole cervix intact such that mechanical strength due to its unique toroid shape was accurately assessed. This 
is contrary to previous mechanical studies, where tests were conducted on excised cervical tissue strips of 4 to $10 \mathrm{~mm}$ in length [57]-[59]. In our experiments, compression was applied along the plane perpendicular to the neck of the cervix at three locations evenly spaced from the cervical os to the edge of the cervix, as shown in Figure 9 (left). Indentations were made in 2-3 mm increments at each location along the cervix. Specifically, the linear actuator was programmed to indent the tissue at $1.7 \mathrm{~mm}, 4.6 \mathrm{~mm}, 7.8 \mathrm{~mm}$ and $9.9 \mathrm{~mm}$. Assuming a cervical length of $2 \mathrm{~cm}$ [15], the compressive values correspond to an axial strain range of 0.09 to 0.49 . Ten compressions at each pre-programmed distance were applied to each of the three locations, for a total of 120 measurements conducted per tissue specimen.

As part of the documentation process and for possible creation of anatomically accurate 3D-printed cervix phantoms in a future project, anatomical features of the tissue specimens were characterized with the NextEngine 3D Scanner shown in Figure 12. As shown in Figure 13, the specimen was placed onto a turntable, with the neck of the cervix supported to ensure the perpendicular surface of the cervix was captured by the scanner. Two scans were performed for each specimen to capture both the anterior and posterior views of the tissue. The turntable was programmed to rotate $360^{\circ}$ in $36^{\circ}$ increments. The $3 \mathrm{D}$ scan was processed in an open-source mesh processing software system MeshLab using Poisson surface reconstruction. MeshLab's Poisson surface reconstruction was selected due to accessibility to the software and its ability to generate a watertight mesh from the surface sample points suitable for 3D printing.

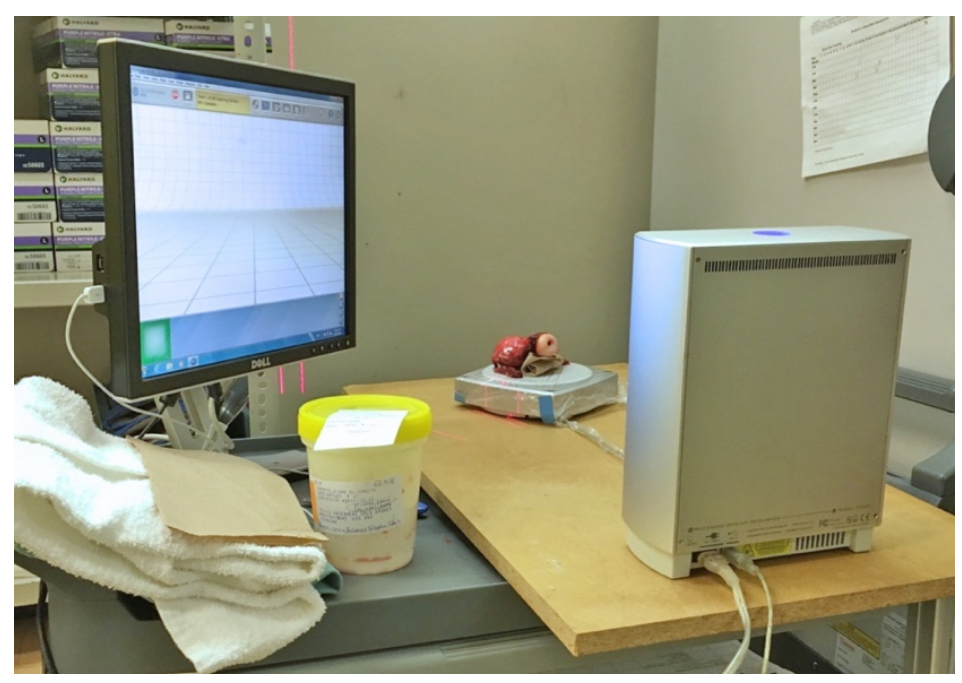

Figure 12: The experimental setup of the NextEngine 3D Scanner being used to characterize the topological features of the tissue specimen 


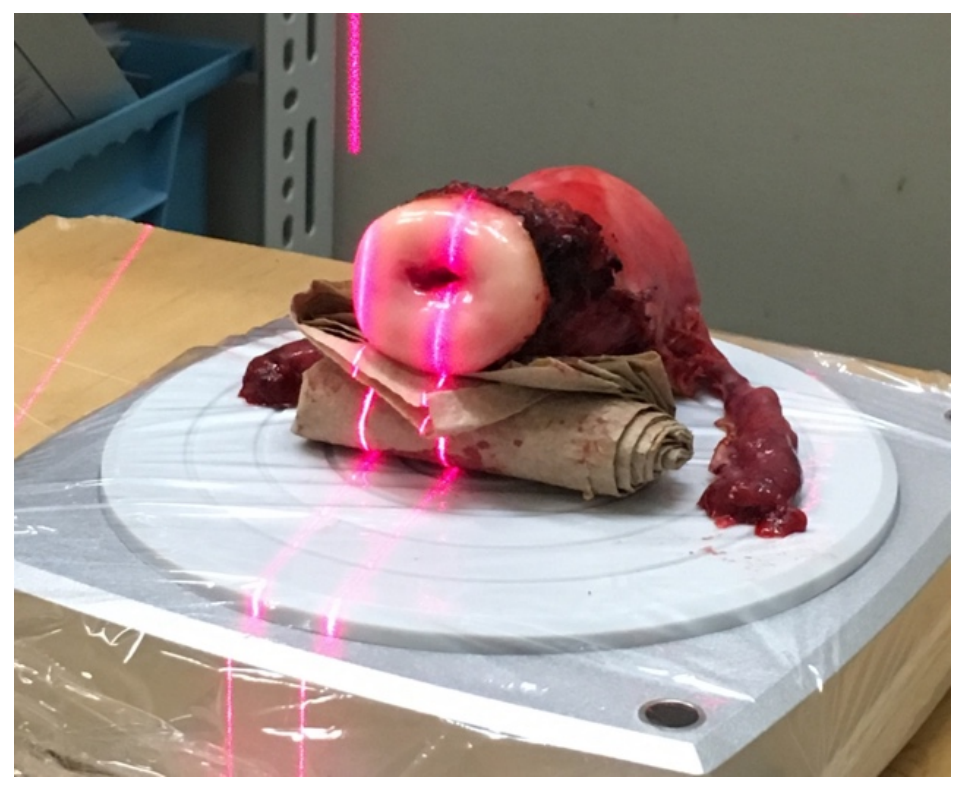

Figure 13: Post-hysterectomy ex vivo uterus and cervical tissue sample tested at McMaster Health Sciences Centre (Hamilton, ON)

\subsection{Design and Characterization of the Cervical Phantom}

The following section describes the design and characterization of the cervix phantom prototype. The cervix phantom utilizes particle jamming technology to achieve softening and passive dilation and effacement characteristic of the cervix during latent labour. Efficacy of this prototype was validated through mechanical testing and feedback of volunteers. Additionally, the system was extended to study its ability to passively dilate and efface in response to labour induction.

\subsubsection{Design of the Phantom}

The cervix phantom prototype, shown in Figure 14, was created using a non-porous, flexible toroid-shaped Qualatex 16" latex balloon loosely packed with 35 g of granular Melitta Arabica coffee grounds. The commercial off-the-shelf balloon was selected for its toroidal geometry, which is similar to the cervix with an opening representative of the cervical os. The balloon has a outer diameter of $10 \mathrm{~cm}$, and inner diameter of $2 \mathrm{~cm}$. Coffee grounds were used to fill the balloon, as they are reported to have a high strength-to-weight ratio in comparison to other common granular 
materials and hence commonly used for particle jamming applications [49]. The balloon was secured onto a metal frame using four evenly spaced magnets attached onto the frame and four dimes secured onto the corresponding inside layer of the balloon.

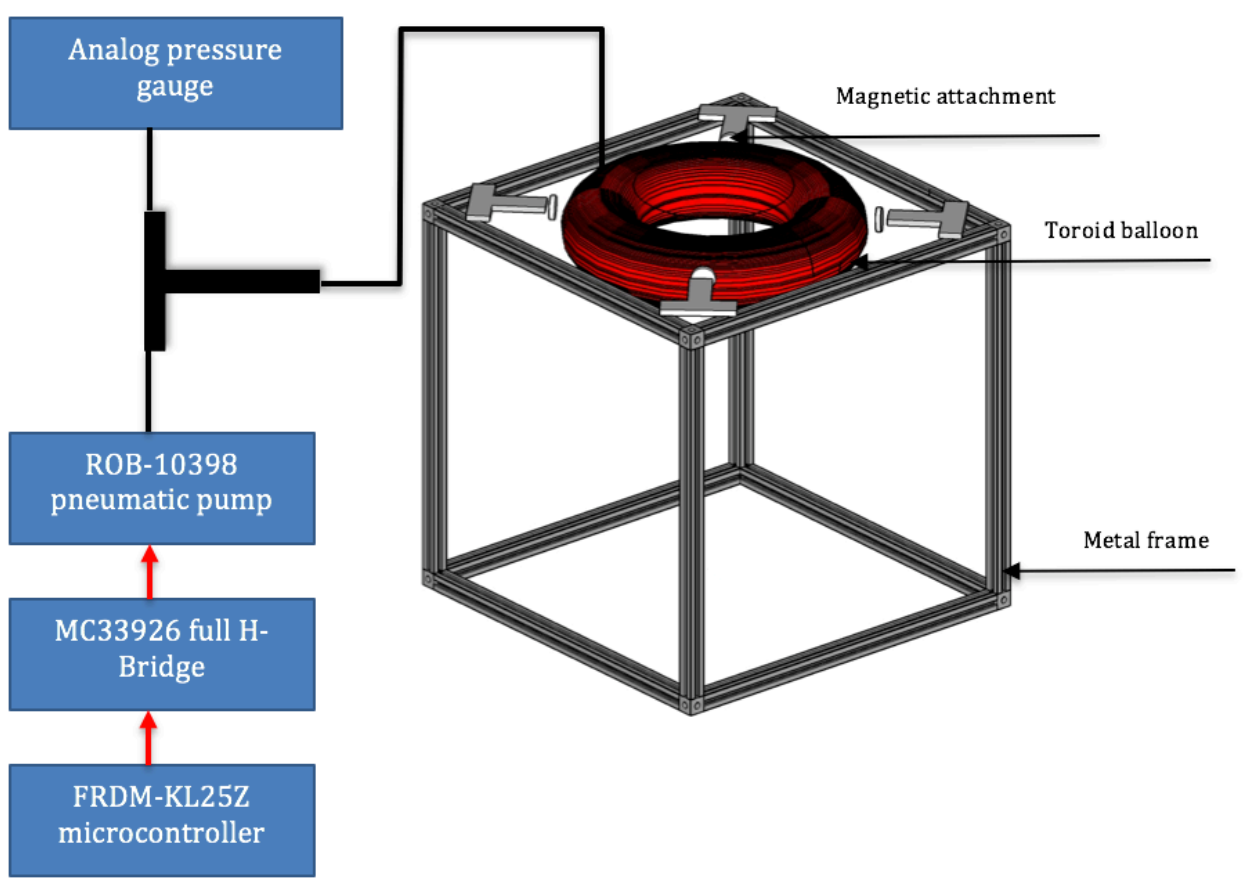

Figure 14: The cervix phantom prototype

Dynamic compliance of the cervix phantom was achieved through the adaptation of pneumatic particle jamming theory. Specifically, the Sparkfun ROB-10398 pneumatic pump was attached to the opening of the balloon to vary the vacuum pressure inside the cervix balloon. In accordance with particle jamming theory, the coffee grains flowed at atmospheric pressure when excess interstitial fluid (i.e. air) was present, but formed a compact, hard solid when a vacuum was applied to removed the fluid [12]. The pneumatic pump was controlled using the Freescale FRDMKL25Z microcontroller and Freescale MC33926 full H-Bridge. The vacuum pressure was measured using the Omega engineering analog pressure gauge PGU-20L-30PSI/2BAR connected through a T-connector to the cervix balloon and pneumatic pump. 


\subsubsection{Compliance of the Phantom}

Vacuum pressures ranging from $50.8 \mathrm{mmHg}$ to $406.4 \mathrm{mmHg}^{2}$ were applied to the cervix balloon to simulate compliance ranging from hard to soft. The vacuum pressure range applied was in accordance with values reported in particle jamming literature, where maximum vacuum pressures of 563 to $600 \mathrm{mmHg}$ were applied [49], [51]. The testing platform used to test the strength of the ex vivo post-hysterectomy tissue was adapted to determine the compliance of the phantom, as shown in Figure 15. The cervix phantom was secured on the OHAUS Scout Pro SP2001 scale, and the 3D printed case and MicroRAX frame housing the Firgelli L12 linear actuator was lowered to indent the cervix phantom with the same $4.5 \mathrm{~mm}$ diameter ABS tip. The cervix balloon was indented by pre-programmed distances of $1.7 \mathrm{~mm}, 4.6 \mathrm{~mm}, 7.8 \mathrm{~mm}$ and $9.9 \mathrm{~mm}$ at vacuum pressures of $0 \mathrm{mmHg}, 101.6 \mathrm{mmHg}, 203.2 \mathrm{mmHg}, 304.8 \mathrm{mmHg}$ and $406.4 \mathrm{mmHg}$. The force corresponding to each displacement and vacuum pressure setting was determined. Ten trials were conducted for each displacement and vacuum pressure setting, for a total of 200 trials.

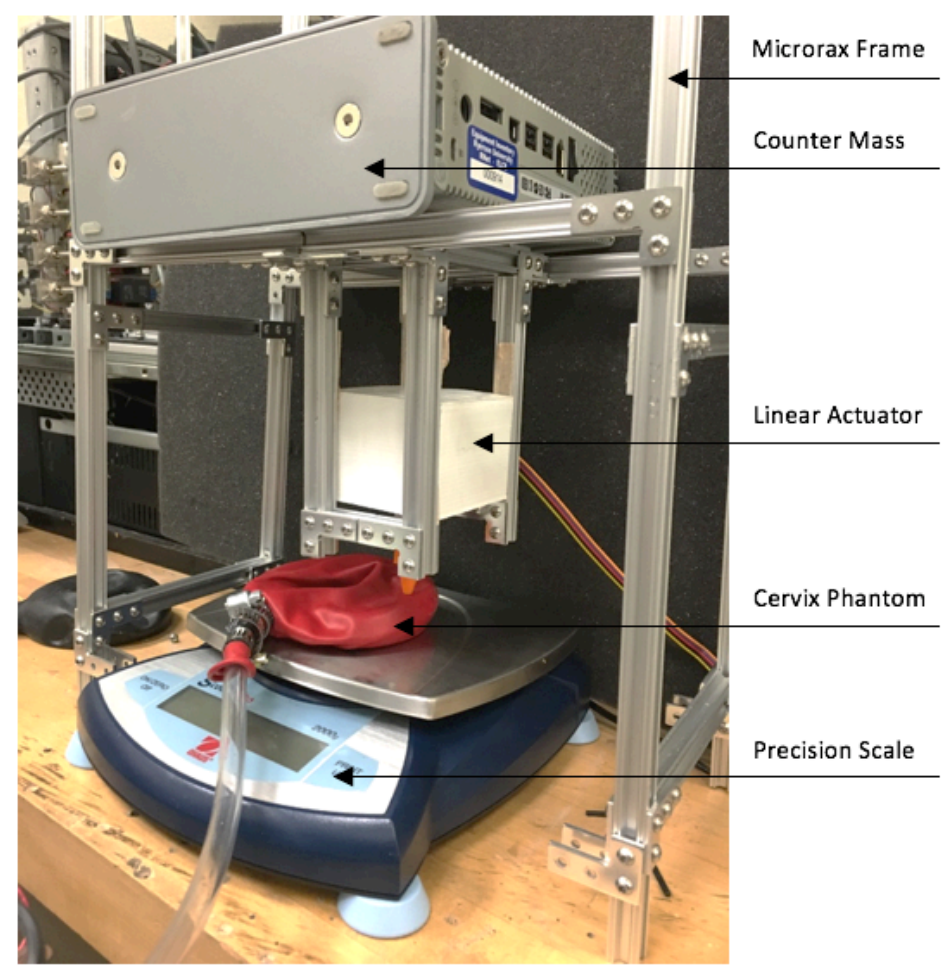

Figure 15: The testing platform was adapted to test the compliance of the cervix phantom prototype

\footnotetext{
${ }^{2}$ Values are derived from unit conversion of inHg (analog gauge reading) to $\mathrm{mmHg}$
} 


\subsubsection{Validation of the Phantom through Tactile Psychophysics Experiments}

The cervix model was validated through feedback from 10 inexperienced volunteers. As the phantom will ultimately be used to train students, volunteers without prior experience in labour and delivery were selected. Participants were asked to identify the transition of the system from "hard" to "soft" as the vacuum pressure decreased from $406.4 \mathrm{mmHg}$ to $0 \mathrm{mmHg}$ in order to determine the threshold between these two states. Ten trials were conducted for each participant. After the threshold was determined, the same participants were asked to feel the firmness of the model at randomized vacuum pressures and asked to identify whether it felt "hard" or "soft" such that the system specificity both above and below the threshold could be determined. As shown in Figure 16, participants wore a pair of Beats Solo 2 on-ear headphones with white noise from the Simply Noise online generator (https://simplynoise.com/) playing in order to ensure that the sound of the pressure pump of the model could not be heard during the experiment. Similarly, the system was covered with a cloth to ensure that the appearance of the model would not bias the responses.

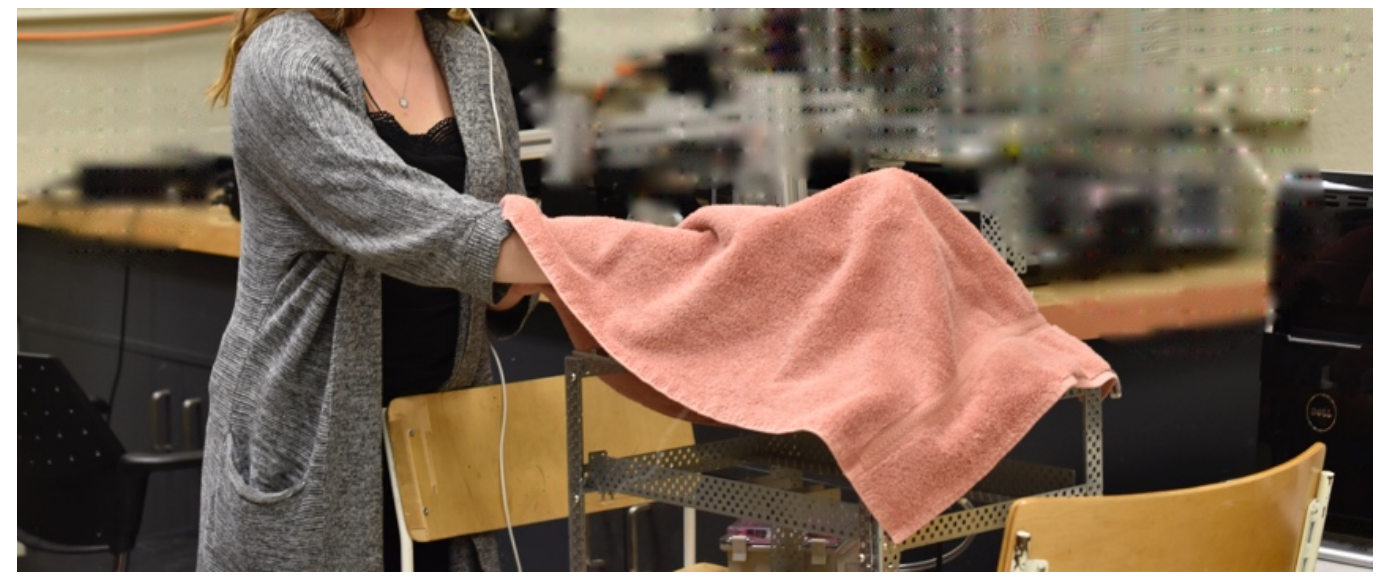

Figure 16: Participant interacting with the cervix phantom prototype in psychophysics experiment

\subsection{Mechanical Labour Induction}

The cervix phantom prototype was extended to study its response to mechanical labour induction using a Foley catheter. Clinically, a Foley catheter is inserted through the cervical canal into the extra-amniotic space, as shown in Figure 17. In our cervix phantom, this was simulated through placing a small dome with an inelastic cable on top of the inner circle of the toroid such that a mass could be suspended from the cervix balloon, as shown in Figure 18. The passive dilation and 
effacement of the cervix model in response to applied loads of $500 \mathrm{~g}, 750 \mathrm{~g}$ and $1000 \mathrm{~g}^{3}$ were determined for applied vacuum pressures ranging from $50.8 \mathrm{mmHg}$ to $406.4 \mathrm{mmHg}$. Load values were selected based on the fact that tractive force applied during catheter balloon labour induction is typically $500 \mathrm{~g}$ or more [60].

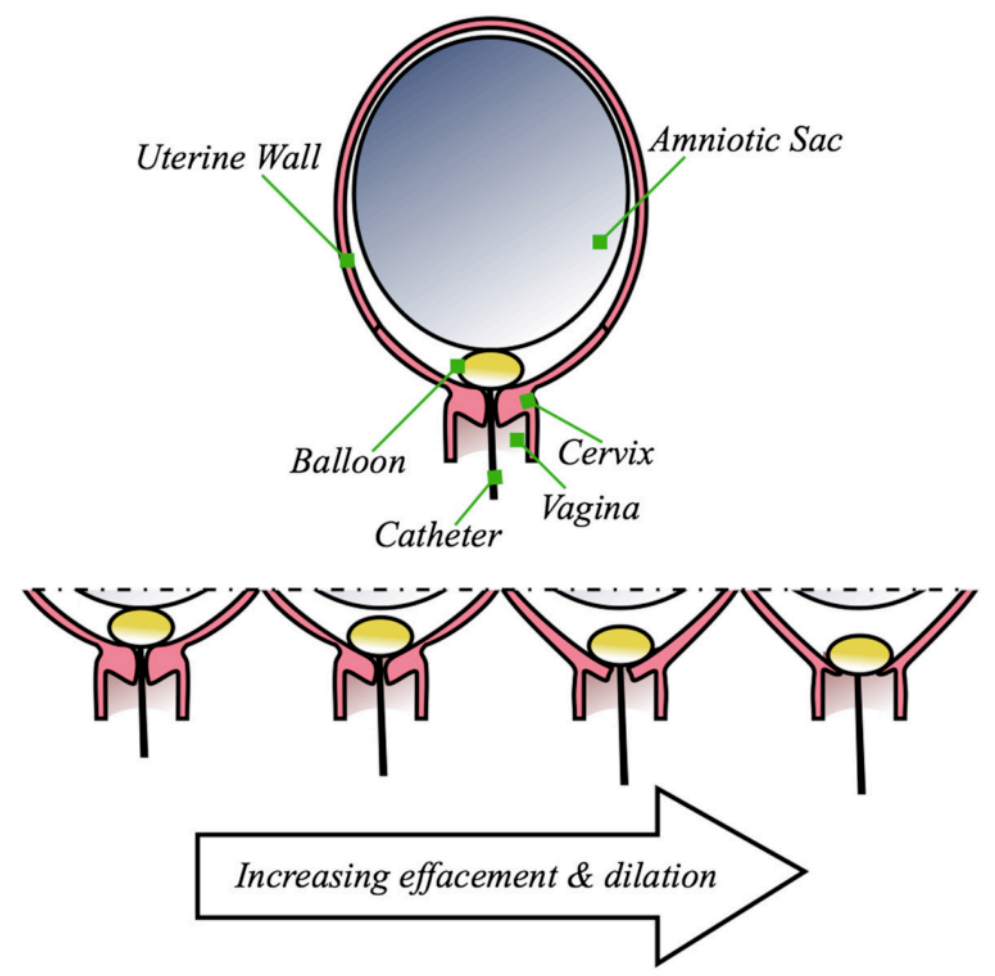

Figure 17: The use of a Foley catheter to induce labour. From Smith, 2013 [61]

${ }^{3}$ Corresponds to force of $4.9 \mathrm{~N}, 7.4 \mathrm{~N}, 9.8 \mathrm{~N}$ respectively 


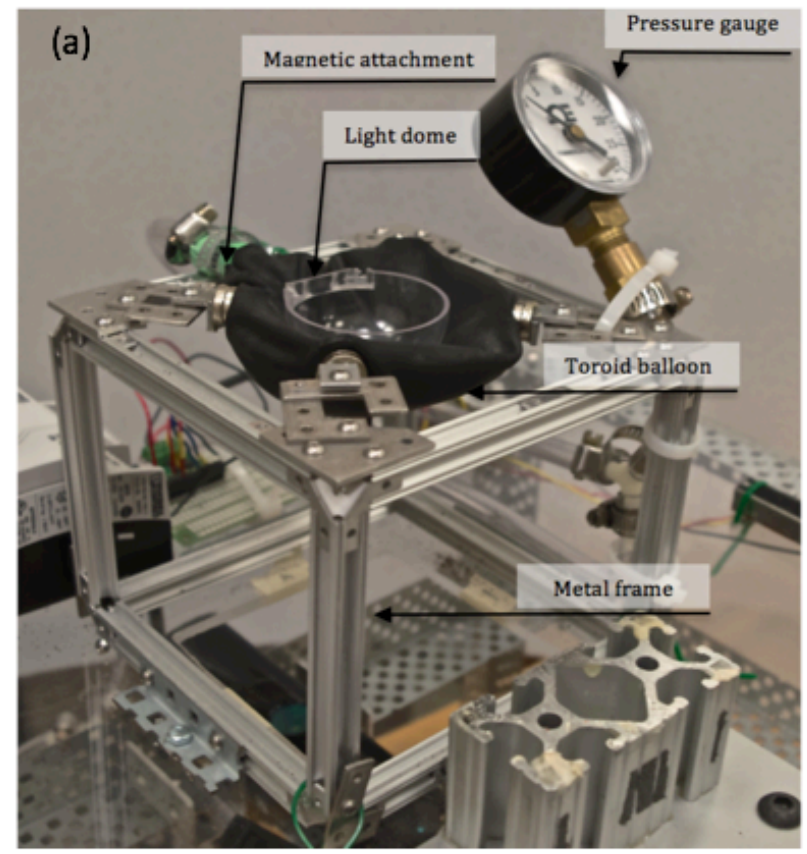

(b)

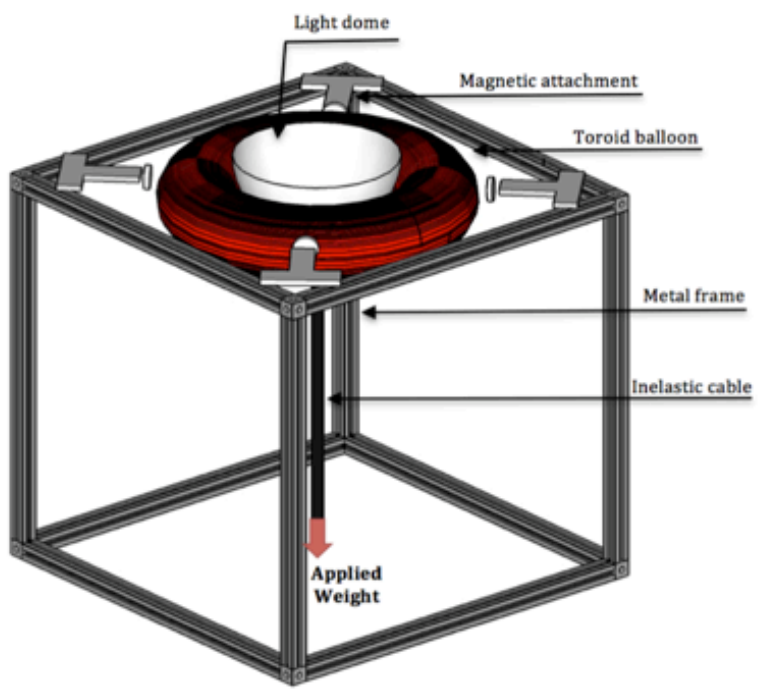

(c)

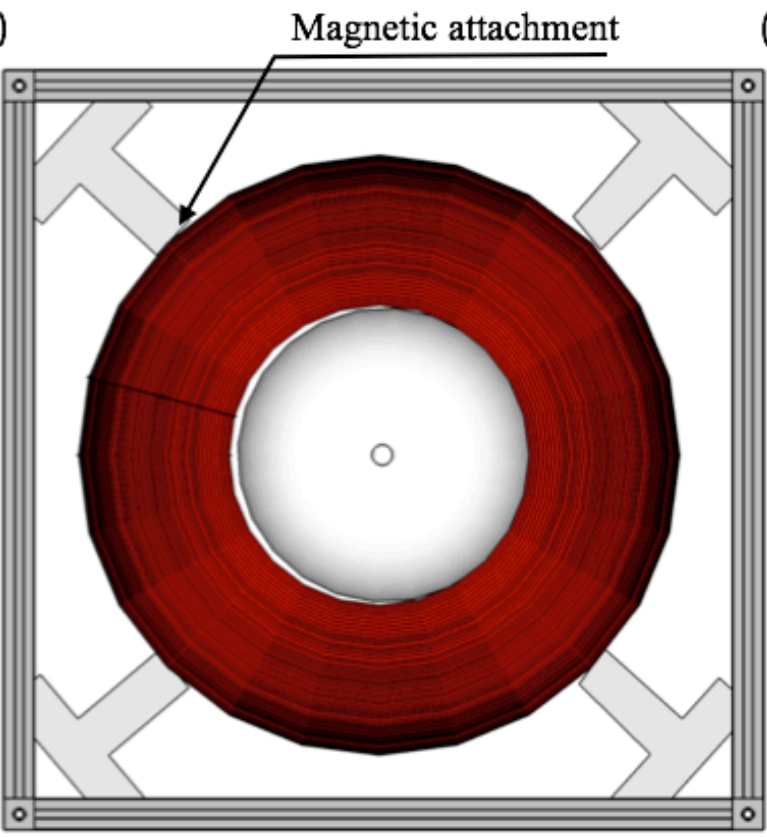

(d)

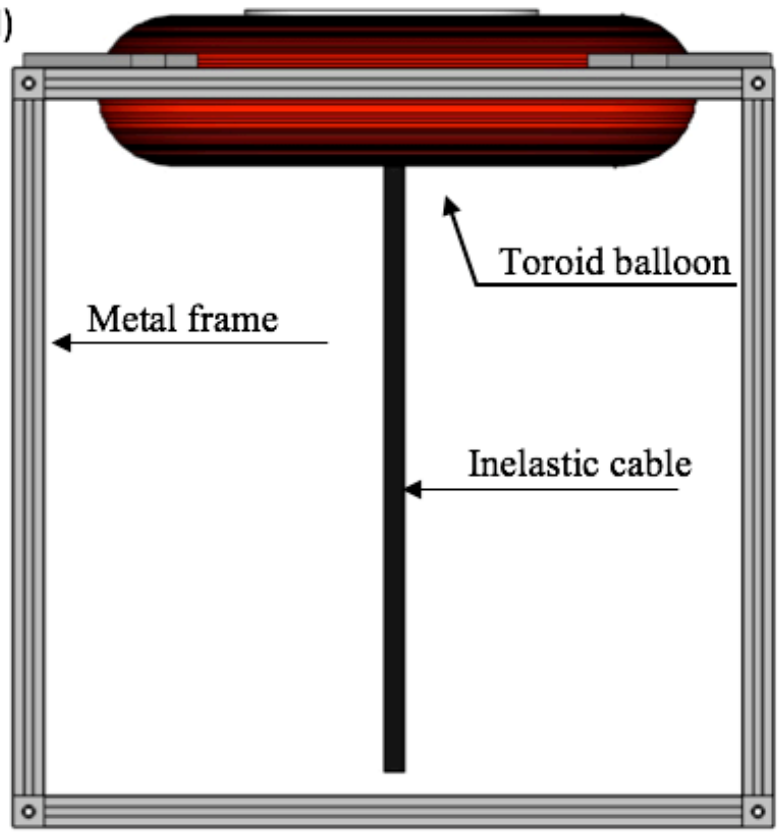

Figure 18: Representations of the cervix phantom prototype: (a) the actual cervix prototype, (b) the simplified model and (c) elevated and (d) cross-sectional views. 


\subsubsection{Optical Measurement using Kinect Cameras}

Dilation and effacement were measured using optical sensors of two Kinect cameras positioned below (providing an elevated view) and in front (providing a cross-sectional view) of the model, as shown in Figure 18 (c) and 18 (d), respectively. The Kinect cameras were initially selected for their depth perception features, however this feature was found to not be sensitive enough to measures volumetric changes related to effacement and dilation. Therefore, only the Kinect's standard RGB images were used to measure dilation and effacement. The resulting images were processed in MATLAB R2015 using the "imtool" and "roipoly" functions of the Image Processing Toolbox. The Kinect cameras were calibrated using small plastic Lego bricks placed in the plane of measurement for both cameras. A standard $1.6 \mathrm{~cm}$ x $1.6 \mathrm{~cm}$ Lego brick was used for the crosssectional camera, while a standard $1.6 \mathrm{~cm} \mathrm{x} 4.8 \mathrm{~cm}$ Lego brick was used for the elevated camera. The images from both cameras views were captured such that the number of pixels corresponding to the length and width of the plastic blocks could be determined using the "imtool" function of MATLAB R2015. For each camera, the average of the length and width measurements were taken to determine the average pixels $/ \mathrm{mm}$.

\subsubsection{Measuring Passive Effacement and Dilation}

The step-by-step experimental procedure used to measure the effacement and dilation are detailed in the following:

1. The position of the cervix balloon was initialized using a box flush against the bottom of the balloon. The vacuum pressure was set to $406.4 \mathrm{mmHg}$ vacuum. The dome without any suspended mass was placed on top of the cervix balloon.

2. Kinect images were taken for both the cross-sectional and elevated cameras prior to the addition of the mass. This served as the initial position.

3. A $500 \mathrm{~g}$ weight, representative of a typical mass used for induction with a Foley catheter [60], was suspended from the dome. The amount of dilation and effacement in response to the mass was recorded from Kinect images were taken for both the cross-sectional and elevated cameras, respectively.

4. The cross-sectional camera images were analyzed using the "imtool" function of the Image Processing Toolbox in MATLAB R2015 to determine the change in descent and deflection in response to the applied mass. Effacement was indirectly measured from descent and 
deflection, as shown in Figure 19.

5. The elevated camera images were analyzed using the "roipoly" function of the Image Processing Toolbox in MATLAB R2015 to determine the change in the balloon inner area, which represents the internal os of the cervix. Figure 20 shows the output of the "roipoly" function. The change in diameter (i.e. dilation) of the cervix balloon was indirectly determined by approximating the shape of the inner os to be a circle.

6. The obtained values (in pixels) were converted to millimeters using the average pixels $/ \mathrm{mm}$ values for the respective cameras.

7. Steps 1-4 were repeated for applied masses of $750 \mathrm{~g}$ and $1000 \mathrm{~g}$ and vacuum pressures of $50.8 \mathrm{mmHg}, 76.2 \mathrm{mmHg}, 101.6 \mathrm{mmHg}, 152.4 \mathrm{mmHg}, 203.2 \mathrm{mmHg}$ and $304.8 \mathrm{mmHg}$. Five trials were repeated for each mass and pressure combination. A total of 105 trials were conducted.

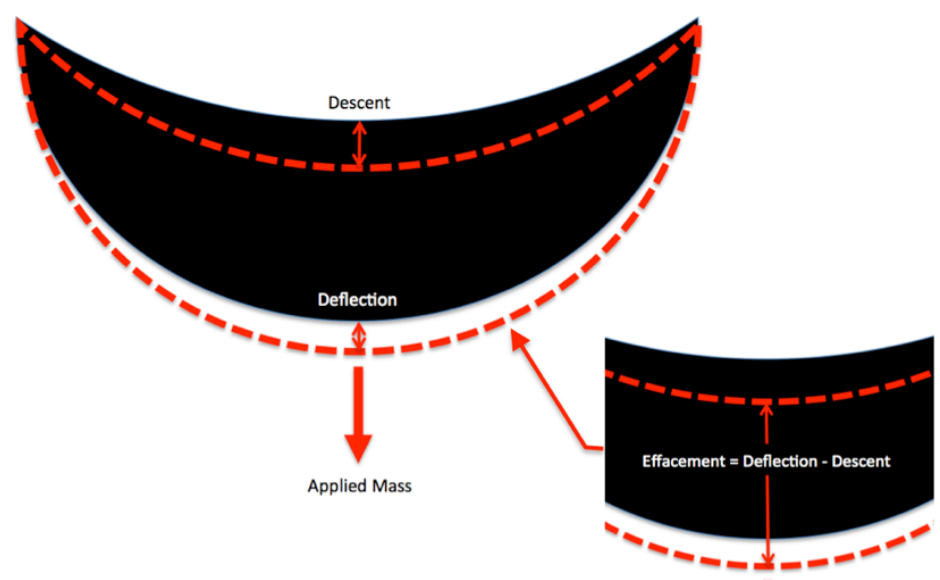

Figure 19: The cross-sectional view of the cervix balloon used to measure the effacement, deflection and descent 


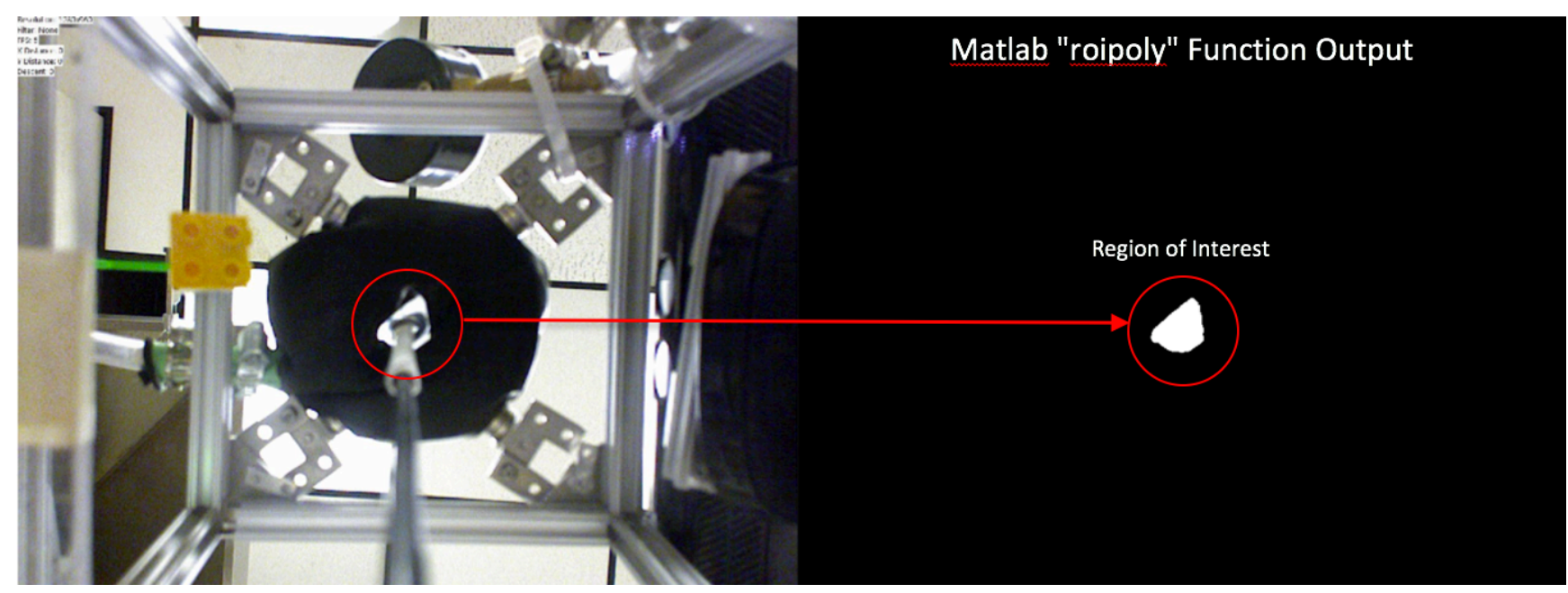

Figure 20: The elevated camera view (left) and "roipoly" function output (right) of the cervix balloon used to measure the dilation 


\section{Results}

\subsection{Characteristics of Ex vivo Post-Hysterectomy Cervical Tissue}

Ten post-hysterectomy uterus and cervix specimens were obtained and tested at the McMaster Health Sciences Centre (Hamilton, ON). Compressive strength, fatigue characteristics and topological features were characterized and summarized in the following section.

\subsubsection{Compressive Strength of Cervical Tissue}

Figures 21, 22 and 23 show the stress-strain curves of individual cervical samples conducted distal, central and proximal to the cervical os, respectively. Ten trials were obtained at the central stress point, while six and eight trials were obtained for the distal and proximal stress points, respectively. Fewer trials were conducted distal to the cervical os, because the diameter of cervix specimens $4,7,8$ and 10 were too small for the probe to be placed without slipping off the edge of the cervix. Similarly, trials could not be conducted proximal to the cervical os for specimens 5 and 10 , because the inner diameter was so large that the probe would slip into the os. Consequently, no meaningful measurements could be obtained in these cases.

While strength of individual cervix specimens varies, trends can be observed in the stressstrain curves. For applied strains from 0 to 0.4 , the stress-strain relationship could be described as linear. For applied strain greater than 0.4, exponential characteristics were observed. Figure 24 compares the compressive strength of different locations along a cervical specimen. Overall, the cervix exhibited the greatest strength close to the cervical os (i.e. 4-325 kPa). At the centre of the cervix, a moderate strength was observed (i.e. 3-275 kPa). Finally, lowest strength was observed close to the edge of the cervix (i.e. 3-180 kPa). Discrepancies between results of individual cervix specimens and reasoning for the observed trends will be explained in the Discussion section. 


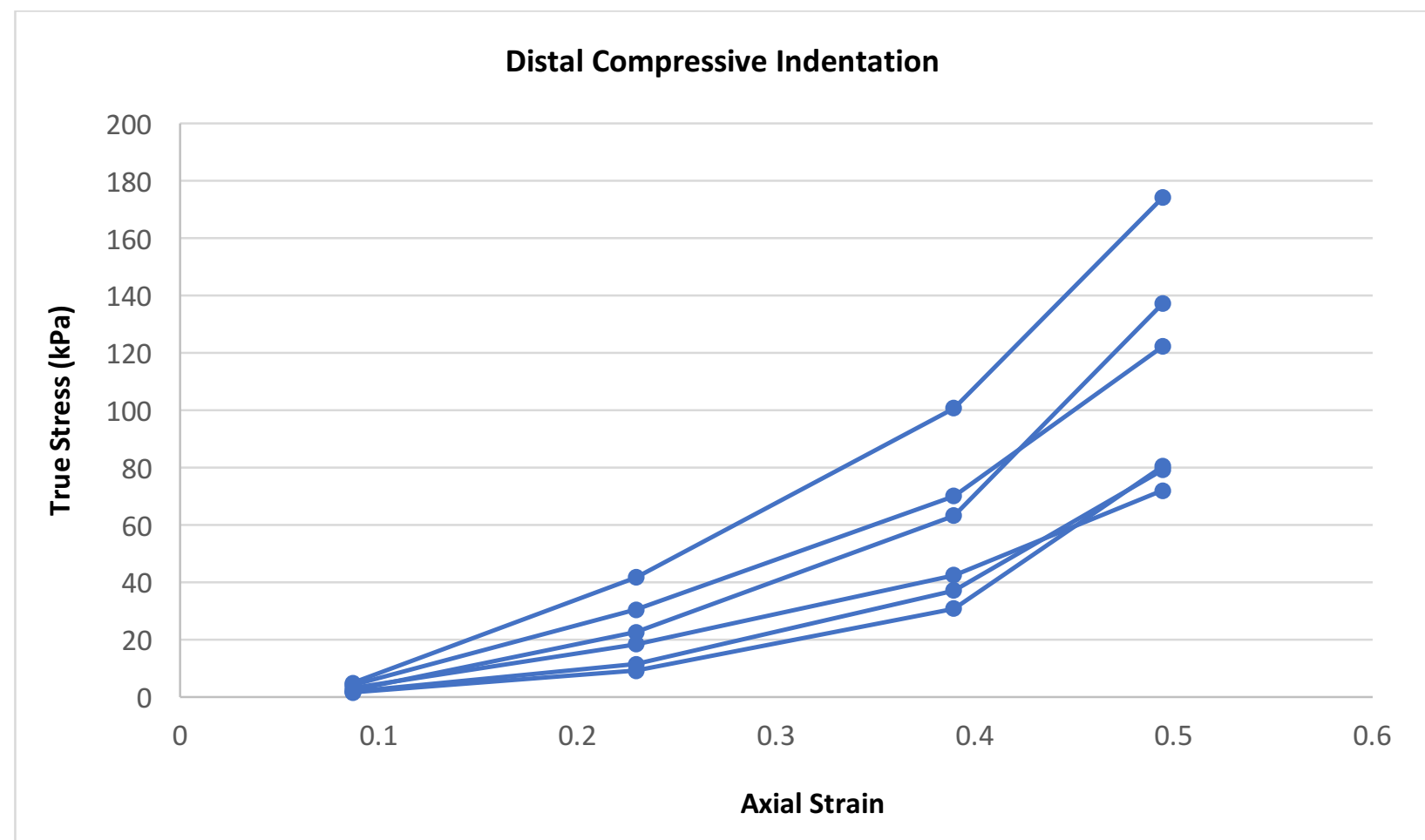

Figure 21: Stress-strain curves of six post-hysterectomy cervical tissue samples tested at a stress point distal to the cervical os

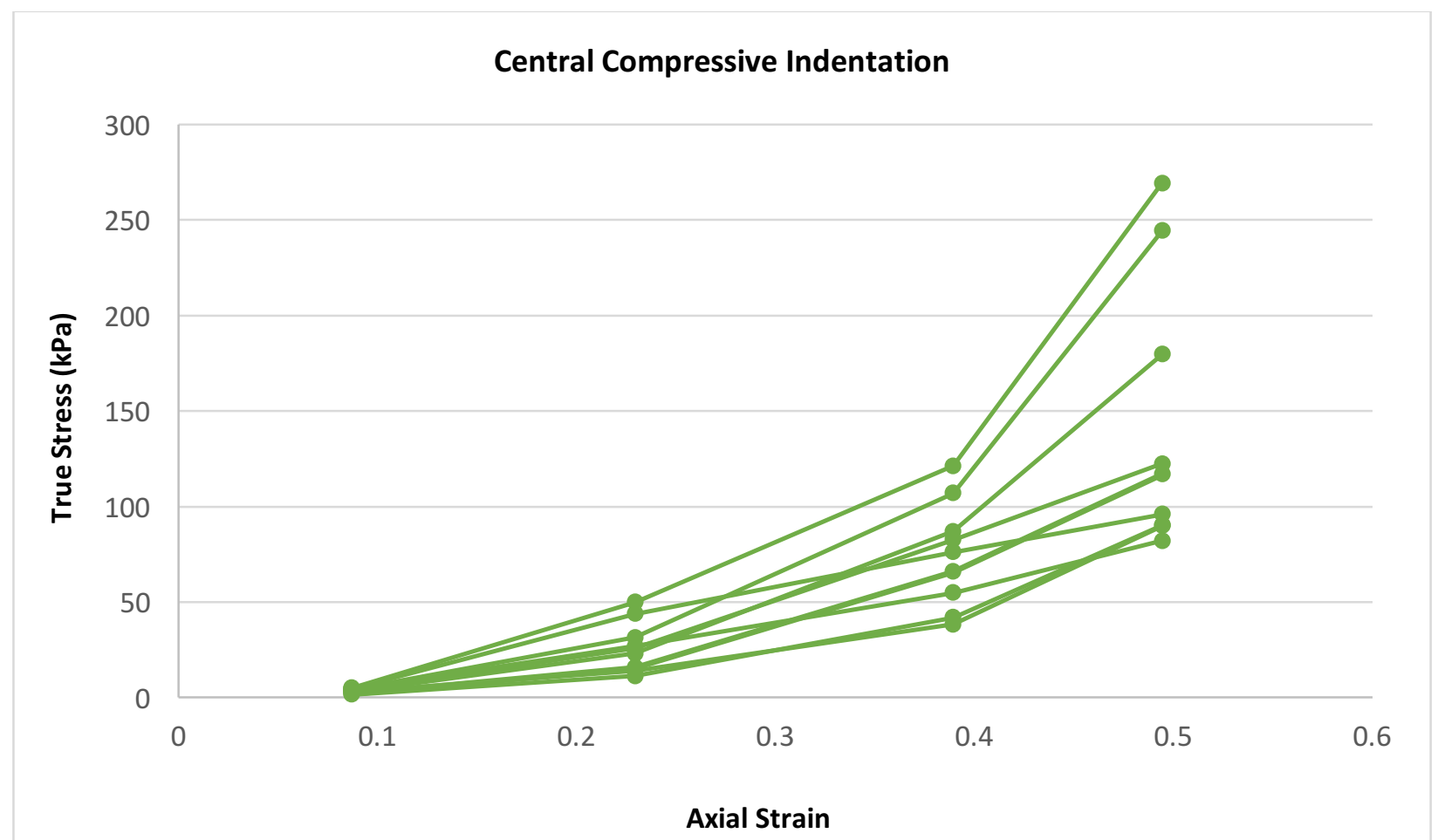

Figure 22: Stress-strain curves of ten post-hysterectomy cervical tissue samples tested at a central stress point 


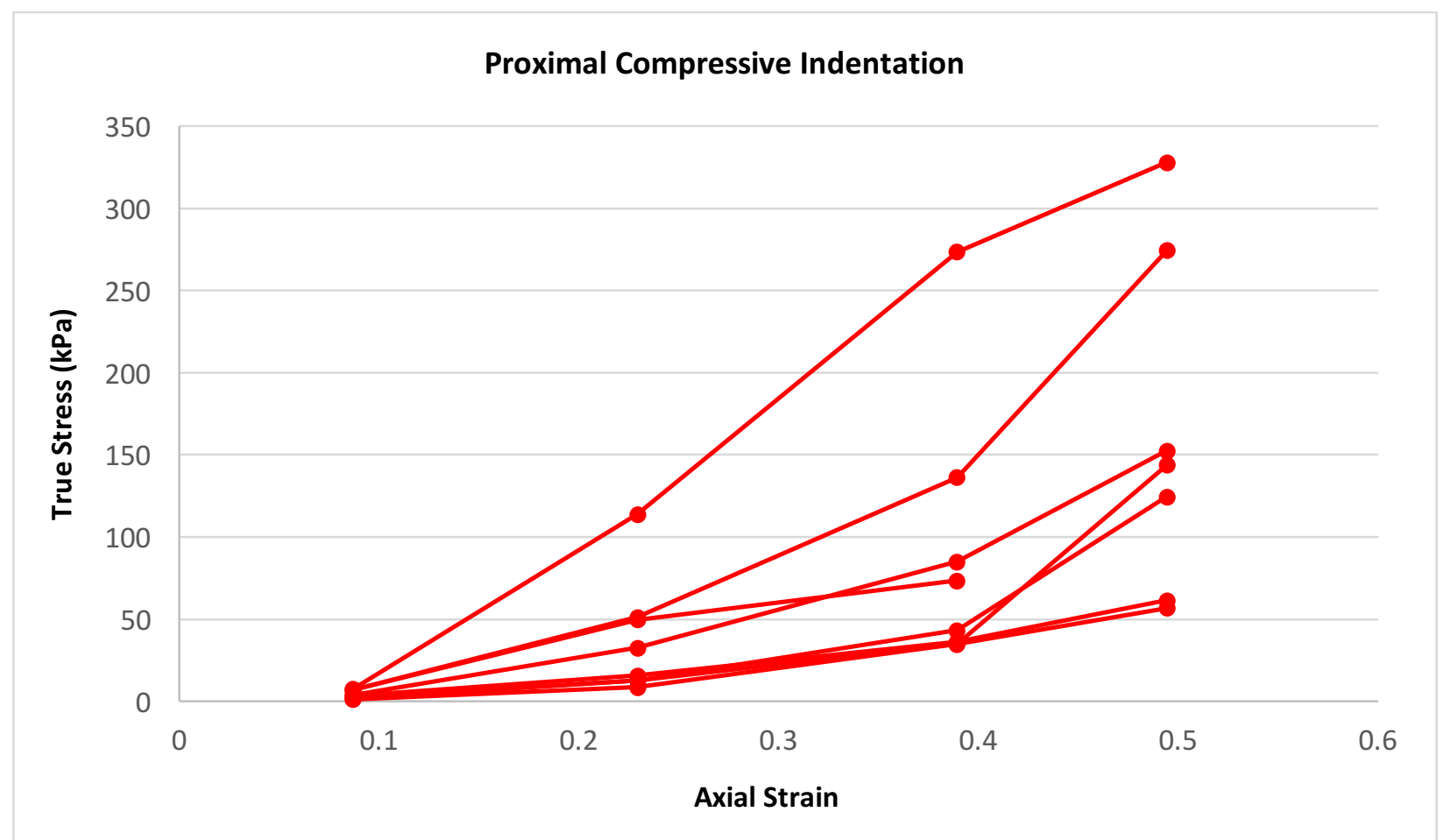

Figure 23: Stress-strain curves of eight post-hysterectomy cervical tissue samples tested at a stress point proximal to the cervical os 


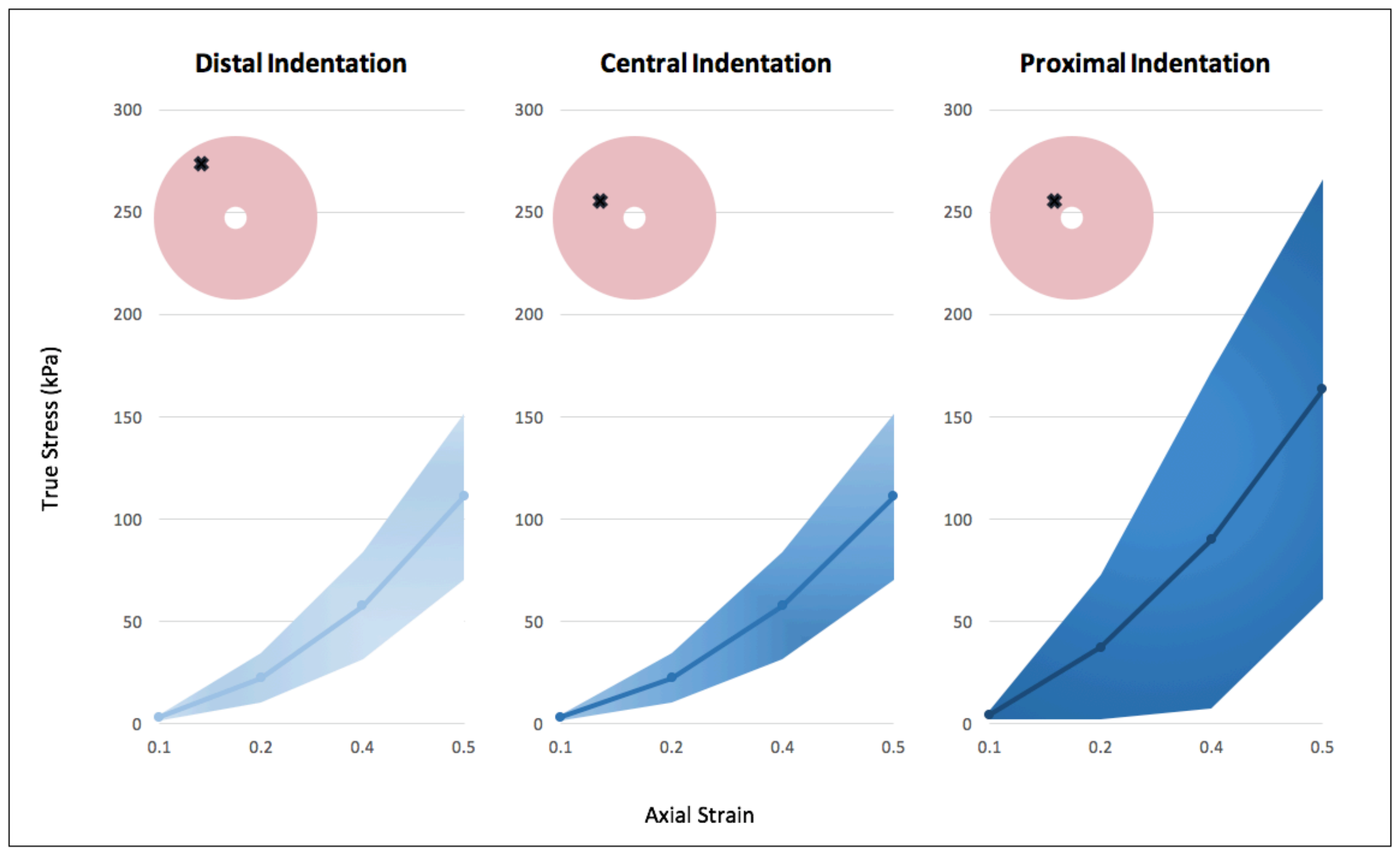

Figure 24: Comparison of cervical tissue compressive indentations conducted at different locations along the axial plane of the cervical tissue sample 


\subsubsection{Fatigue Characteristics of Cervical Tissue}

Figure 25 shows the effect of ten consecutive trials on the cervix tissue compressive strength. At higher strains of 0.4 and 0.5 , a decline in strength can be observed due to weakening caused by repeated applied loading. For lower strains of 0.1 and 0.2 , the tissue recovers from the loading and does not exhibit this behavior.

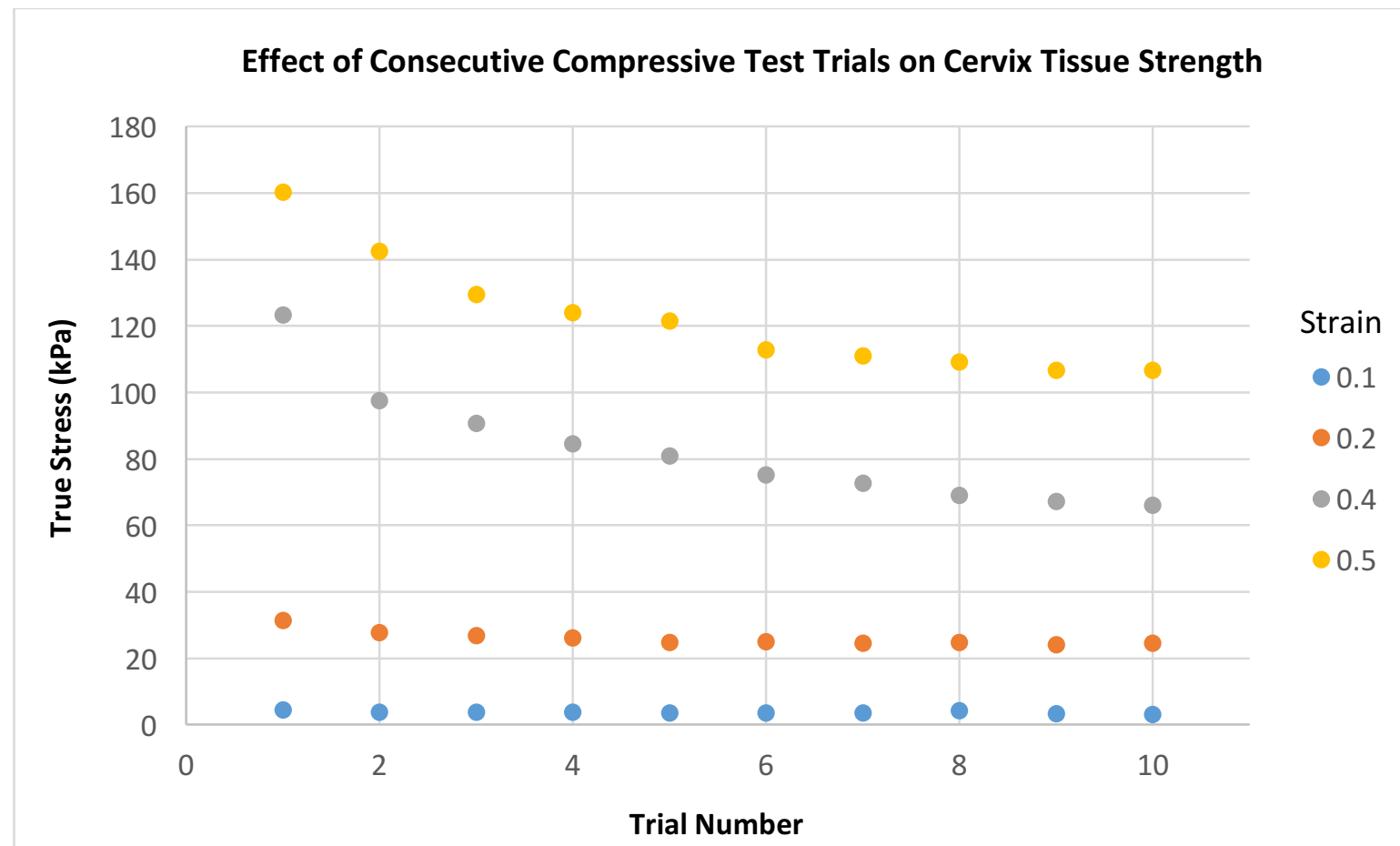

Figure 25: The effect of 10 consecutive trials and strains conducted at a central stress point on cervical tissue strength, lower strains were not affected by continuous loading in comparison to higher strains. 


\subsubsection{Topological Features of Cervical Tissue}

Examination of the topological features of post-hysterectomy cervical tissue revealed that significant differences can be observed between specimens. Figure 26 shows the $3 \mathrm{D}$ scan of two different cervix samples. The specimen on the left has a much larger uterus, and a more contracted and narrow cervix, while the specimen on the right is more representative of typical cervix and uterus specimen. Discrepancies between sample topologies can be attributed to difference in patient background, including age, pregnancy history, height and weight. While differences in topologies exist, it remains important to understand and quantify the anatomy of the cervix and uterus such that they can be incorporated into future training models.

Investigations with 3D scanning and printing technologies have demonstrated the feasibility of obtaining anatomical accuracy in the cervix model. Particularly, the 3D scanned data is representative of the cervix at the onset of labour. 3D scans of post-hysterectomy tissue were taken using the NextEngine 3D Scanner Ultra HD at McMaster Health Sciences Center (Hamilton, ON). Figure 27 (a) and (b) shows the anterior (left) and lateral (right) views of the 3D rendered post-hysterectomy uterus and cervix, respectively. These scans confirm the ability for current scanners to pick up the curvatures and textures of the tissue sample. Holes in the raw 3D scans were filled in using the Poisson surface reconstruction of MeshLab to produce a watertight model suitable for 3D printing, as shown in Figure 27 (c). Figure 27 (d) shows the resulting ABS plastic 3D cervix phantom print using the Lulzbot Taz 4 3D printer [62].

Given available fabrication tools, printing a hollowed cervix phantom in a soft material was infeasible as demonstrated in Kim's thesis [43]. Therefore, in order to achieve dynamic compliance, the present cervix phantom features a commercial latex balloon that was not anatomically accurate. Further work is required to utilize the 3D scanning data acquired in these experiments to the development of a non-porous, flexible cervical phantom that can make use of particle jamming technology to achieve dynamic compliance and anatomical accuracy. This will be elaborated on in the Discussion section. 


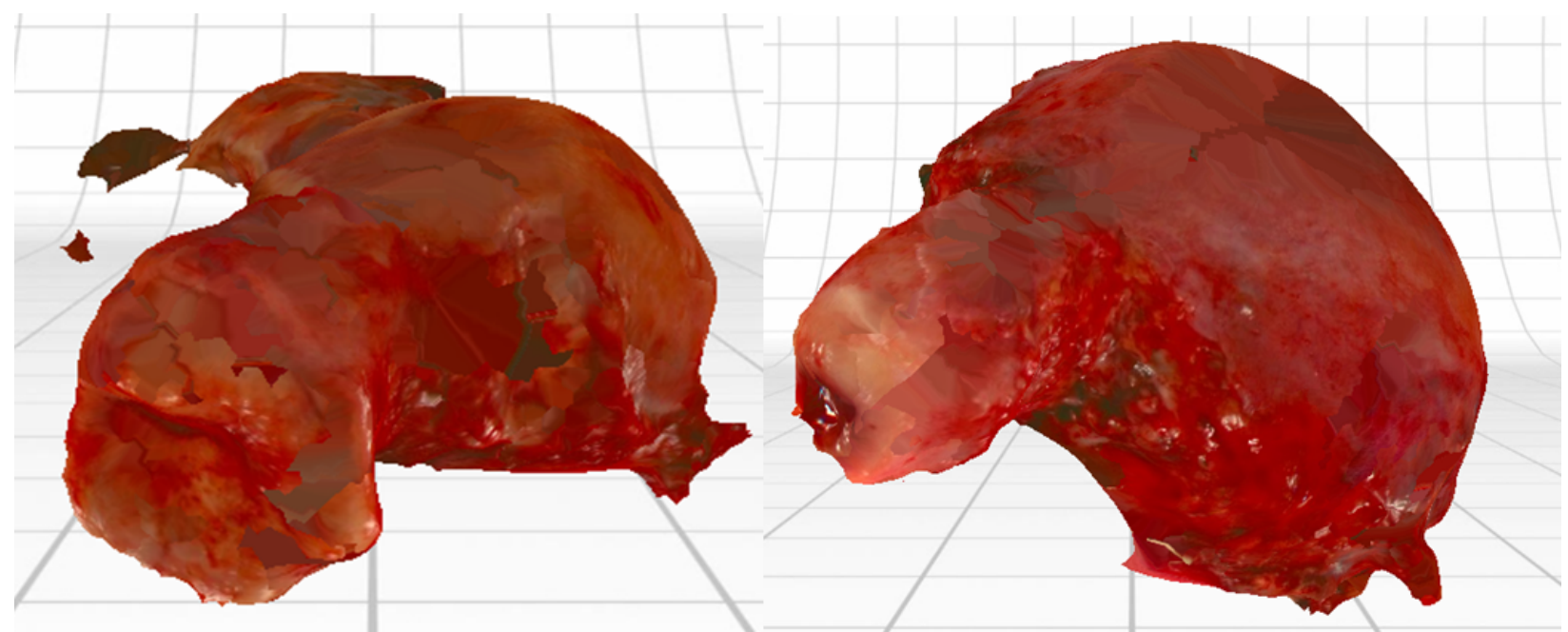

Figure 26: 3D scans contrasting post-hysterectomy sample 6 and 10 


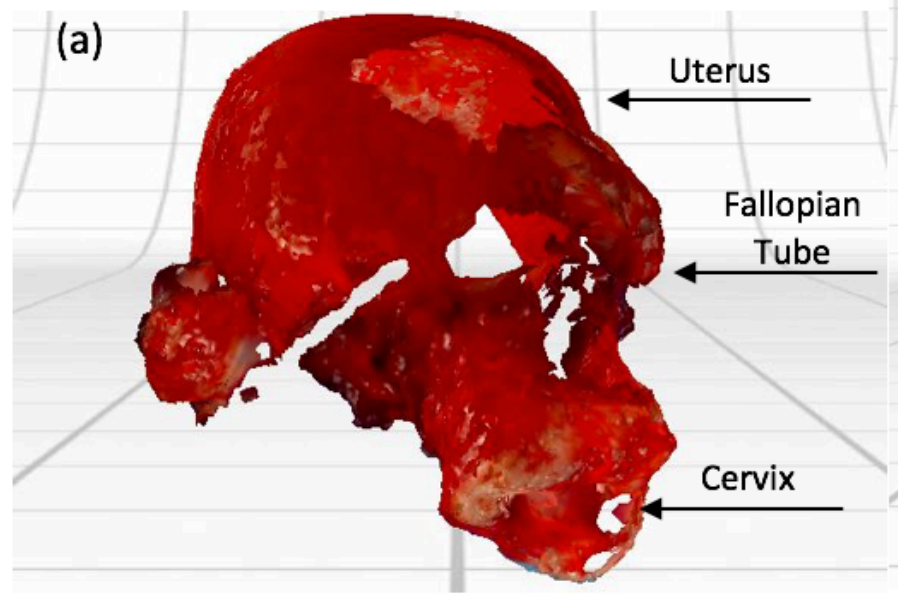

(b)
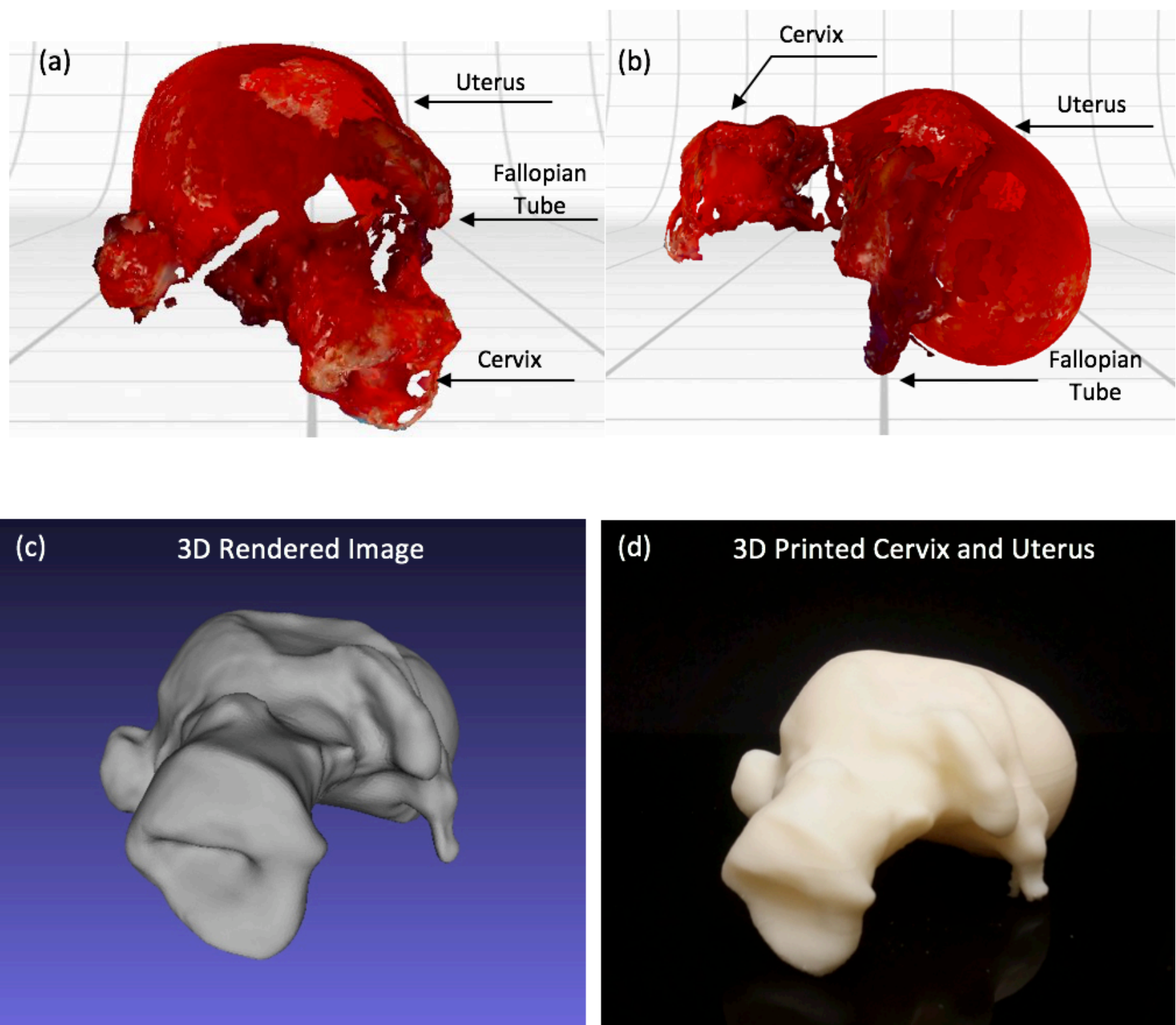

Figure 27: (a) Anterior and (b) Lateral views of a 3D Scanned post-hysterectomy uterus and cervix were obtained and used to produce a (c) watertight processed 3D image and (d) ABS print of the specimen

\subsection{The Cervical Phantom}

The cervix phantom prototype was designed according to the described methodology, and demonstrated to be capable of simulating dynamic compliance in response to varying vacuum pressures. Experimental results characterizing the system are detailed in the following sections. 


\subsubsection{Validation from Psychophysics Experiments}

Responses of volunteers confirmed the palpable change in softness as the vacuum pressure of the cervix phantom prototype was varied. Table 1 summarizes the average vacuum pressure identified as the threshold between "hard" and "soft" for each participant over 10 trials. The average vacuum pressure determined from 10 participants was determined to be $83.3 \mathrm{mmHg}$ ( $\pm 2.67 \mathrm{FS})$. Table 2 summarizes the responses of participants to random vacuum pressure settings between 0 to 406.4 $\mathrm{mmHg}$. Above the average vacuum pressure threshold of $83.3 \mathrm{mmHg}$, 64 out of 70 responses identified the system as being "hard". This corresponds to a hardness specificity of $91.4 \%$. Similarly, below a vacuum pressure of $83.3 \mathrm{mmHg}, 26$ out of 30 responses identified the system as being "soft", corresponding to a softness specificity of $86.7 \%$.

Table 1: The Average Thresholds Identified for Each Participant

\begin{tabular}{|l|l|l|}
\hline Participant & $\begin{array}{l}\text { Threshold } \\
\text { (mmHg vacuum) }\end{array}$ & $\begin{array}{l}\text { Standard Deviation (\%) } \\
\text { (Full Scale 406.4 mmHg) }\end{array}$ \\
\hline 1 & 27.9 & 3.5 \\
\hline 2 & 40.6 & 6.0 \\
\hline 3 & 40.6 & 6.0 \\
\hline 4 & 116.8 & 15.8 \\
\hline 5 & 106.7 & 8.7 \\
\hline 6 & 76.2 & 15.9 \\
\hline 7 & 152.4 & 18.6 \\
\hline 8 & 27.9 & 5.4 \\
\hline 9 & 149.9 & 9.5 \\
\hline 10 & 94.0 & 14.7 \\
\hline
\end{tabular}


Table 2: Summary of Volunteer Responses to Varying Vacuum Pressures

\begin{tabular}{|l|l|l|}
\hline \multirow{2}{*}{$\begin{array}{l}\text { Vacuum } \\
\text { Pressure } \\
\text { (mmHg) }\end{array}$} & \multicolumn{2}{|l|}{ Response (\%) } \\
\cline { 2 - 3 } & Hard & Soft \\
\hline 0.0 & $0 \%$ & $100 \%$ \\
\hline 25.4 & $0 \%$ & $100 \%$ \\
\hline 76.2 & $40 \%$ & $60 \%$ \\
\hline 127.0 & $80 \%$ & $20 \%$ \\
\hline 152.4 & $90 \%$ & $10 \%$ \\
\hline 203.2 & $100 \%$ & $0 \%$ \\
\hline 228.6 & $90 \%$ & $10 \%$ \\
\hline 279.4 & $90 \%$ & $10 \%$ \\
\hline 381.0 & $100 \%$ & $0 \%$ \\
\hline 406.4 & $90 \%$ & $10 \%$ \\
\hline
\end{tabular}

Figure 28 summarizes the results of the psychophysics experiments graphically. The average threshold pressure is identified by the dotted line. It is evident that below this threshold (i.e. to the left of the line), there is a sharp decrease in the number of "hard" responses and increase in the number of "soft" responses. Likewise, above the threshold (i.e. to the right of the line), there is a sharp increase in the number of "hard" responses and decrease in the number of "soft" responses.

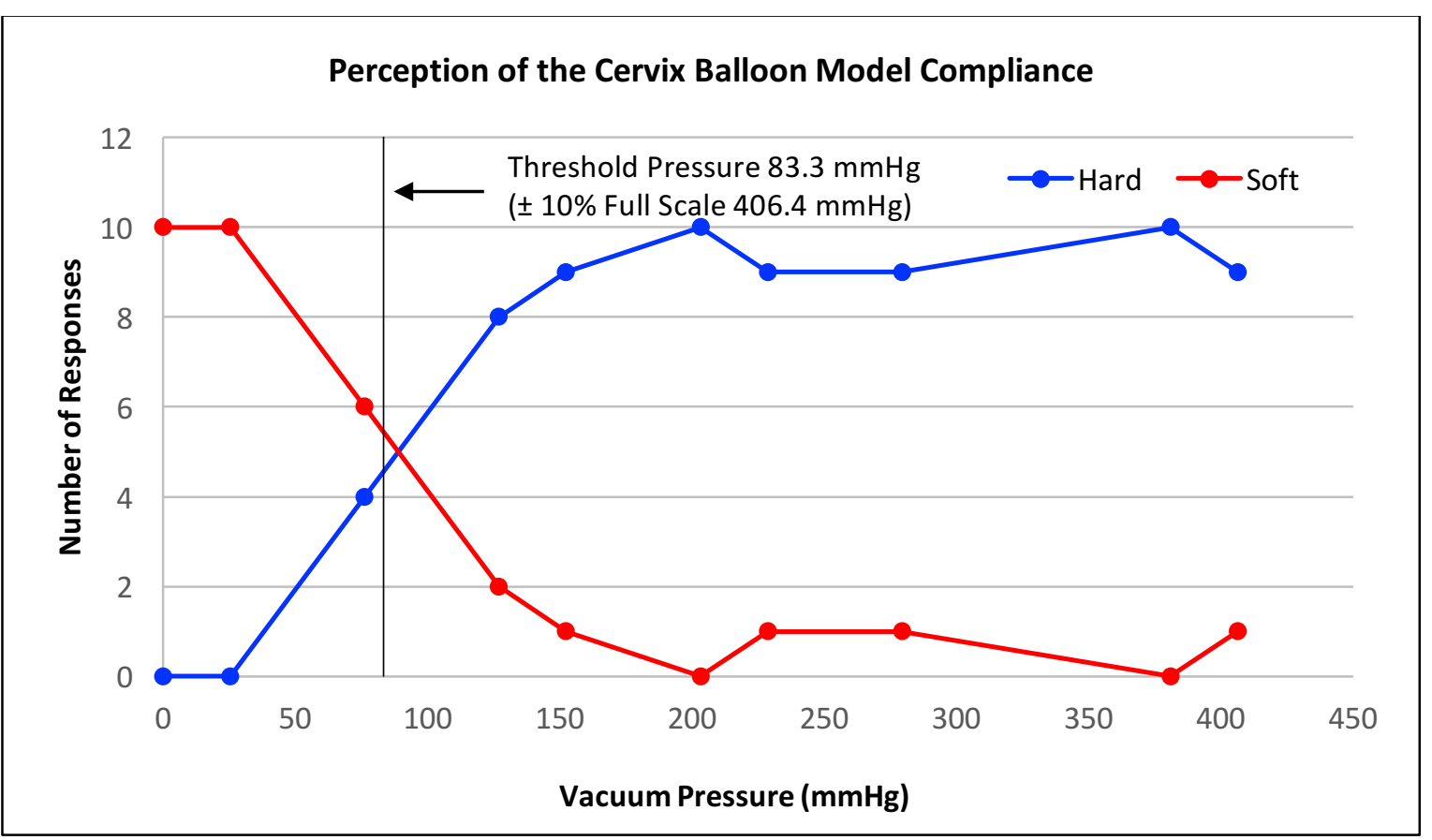

Figure 28: The firmness of the cervix balloon model, as determined by volunteers. At $83.3 \mathrm{mmHg}$ (vacuum), the balloon was perceived to transition from hard to soft 


\subsubsection{Compliance of the Cervix Phantom}

Table 3 summarizes the relationship between stress and strain of the cervix phantom at various vacuum pressures. Results demonstrate the dynamic compliance of the cervix phantom in response to varying vacuum pressures. The phantom is softest at atmospheric pressure, exhibiting strains from 0.09 to 0.50 for stresses between $14.5 \mathrm{kPa}$ and $117.2 \mathrm{kPa}$. As vacuum pressure is increased to $406.4 \mathrm{mmHg}$, the phantom becomes more rigid, with a maximum strain of only 0.23 possible at an applied stress of $419 \mathrm{kPa}$. For higher vacuum pressures, true stress could not be determined at all the pre-programmed strains. The phantom was too rigid at these high vacuum pressures, and the force required to indent tissue by high strains was beyond the capabilities of the linear actuator. These values are outside of the range of interest, as these high forces were never encountered in testing the post-hysterectomy tissue. Therefore, at high vacuum pressures, the phantom is stiffer than human cervical tissue.

Figure 29 shows the stress-strain curve of the cervix phantom prototype at atmospheric pressure (i.e. $0 \mathrm{mmHg}$ ). Similar to the post-hysterectomy cervical tissue, an exponential stressstrain relationship can be observed. Detailed comparisons will be made in the Discussion section.

Table 3: Relationship between Stress and Strain of the Cervix Phantom at Various Vacuum Pressures

\begin{tabular}{|l|l|l|l|l|}
\hline \multirow{2}{*}{$\begin{array}{c}\text { Vacuum } \\
\text { Pressure } \\
(\mathbf{m m H g})\end{array}$} & 0.09 & 0.23 & 0.39 & 0.50 \\
\cline { 2 - 5 } & & & & \\
\hline 0 & $14.5 \mathrm{kPa}( \pm 1.63)$ & $18.8 \mathrm{kPa}( \pm 3.42)$ & $54.6 \mathrm{kPa}( \pm 4.59)$ & $117 \mathrm{kPa}( \pm 12.5)$ \\
\hline 101.6 & $42.3 \mathrm{kPa}( \pm 1.69)$ & $247 \mathrm{kPa}( \pm 15.8)$ & $360 \mathrm{kPa}( \pm 92.3)$ & n.d \\
\hline 203.2 & $36.0 \mathrm{kPa}( \pm 1.38)$ & $269 \mathrm{kPa}( \pm 31.9)$ & $450 \mathrm{kPa}( \pm 47.4)$ & n.d \\
\hline 304.8 & $93.1 \mathrm{kPa}( \pm 1.28)$ & $325 \mathrm{kPa}( \pm 59.3)$ & n.d ${ }^{*}$ & n.d \\
\hline 406.4 & $148 \mathrm{kPa}( \pm 7.03)$ & $419 \mathrm{kPa}( \pm 31.3)$ & n.d & n.d \\
\hline
\end{tabular}

"Not determined, the linear actuator could not indent at high strains when the phantom was too rigid (i.e. at high vacuum pressures) 


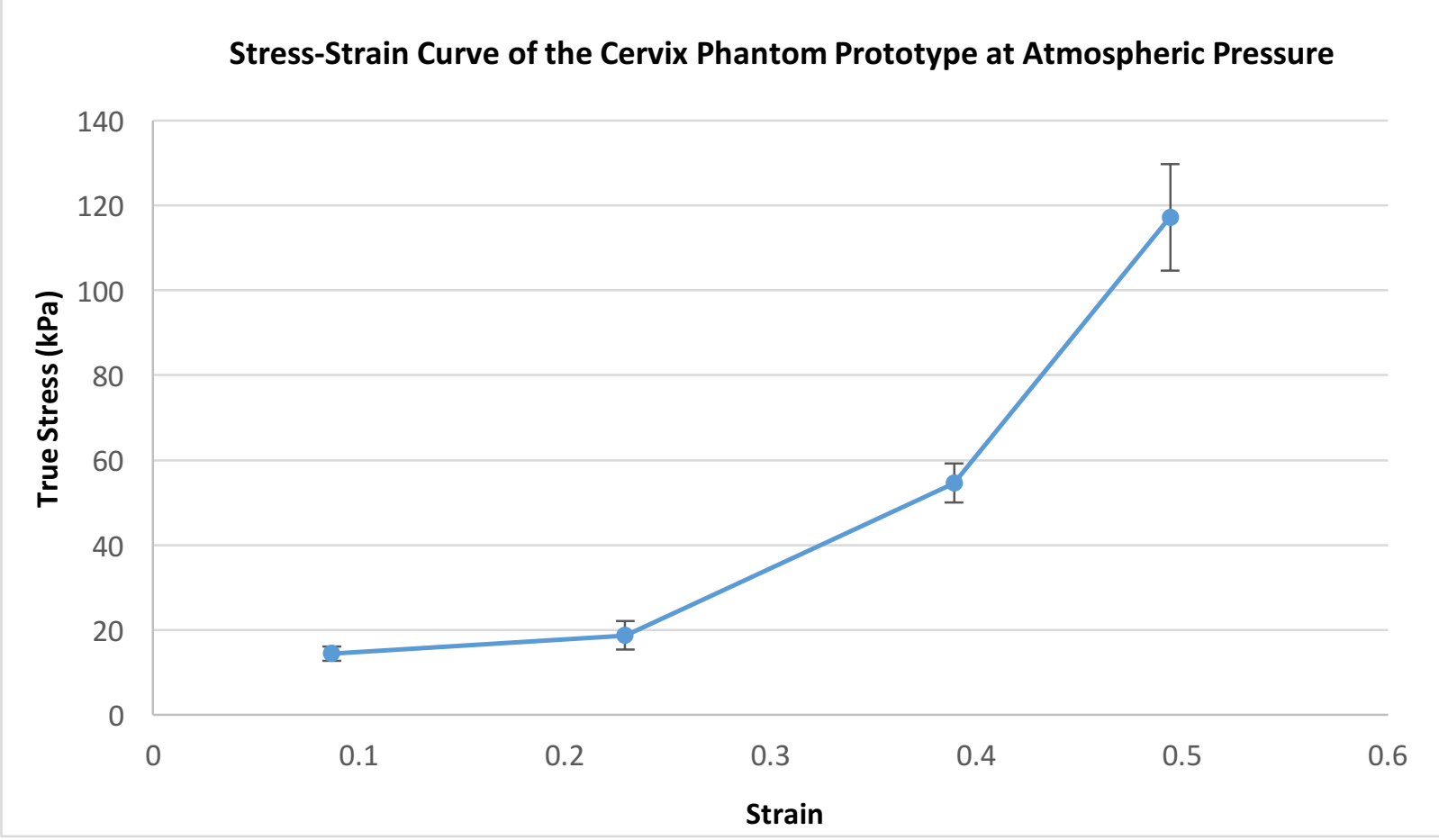

Figure 29: Compressive strength characteristics of the cervix phantom is similar be to the posthysterectomy tissue at atmospheric pressure

\subsection{The Cervical Phantom Response to Labour Induction}

The response of the cervix phantom to an applied tractive force, similar to that applied by a catheter balloon during labour induction, was studied. Experimental results detailing the passive effacement and dilation of the system is described below.

\subsubsection{Passive Effacement, Descent and Deflection}

Figures 30 show the amount of effacement, descent and deflection as the vacuum pressure was decreased from $406.4 \mathrm{mmHg}$ to $50.8 \mathrm{mmHg}$. As demonstrated in Figure 30 (right), the amount of effacement decreases as the vacuum pressure is increased. Similarly, as shown in Figures 30 (left) and 30 (center) respectively, the amount of descent and deflection is observed to decrease drastically between vacuum pressures of $50.8 \mathrm{mmHg}$ and $203.2 \mathrm{mmHg}$. Applied vacuum pressures of greater than $203.2 \mathrm{mmHg}$ was shown to result in minimal descent and deflection. At all values of vacuum pressure, a greater amount of effacement, descent and deflection was observed for the larger applied weights of $1000 \mathrm{~g}$ (green, dotted line) and $750 \mathrm{~g}$ (red, solid line) than the $500 \mathrm{~g}$ applied weight (blue, dashed line). 


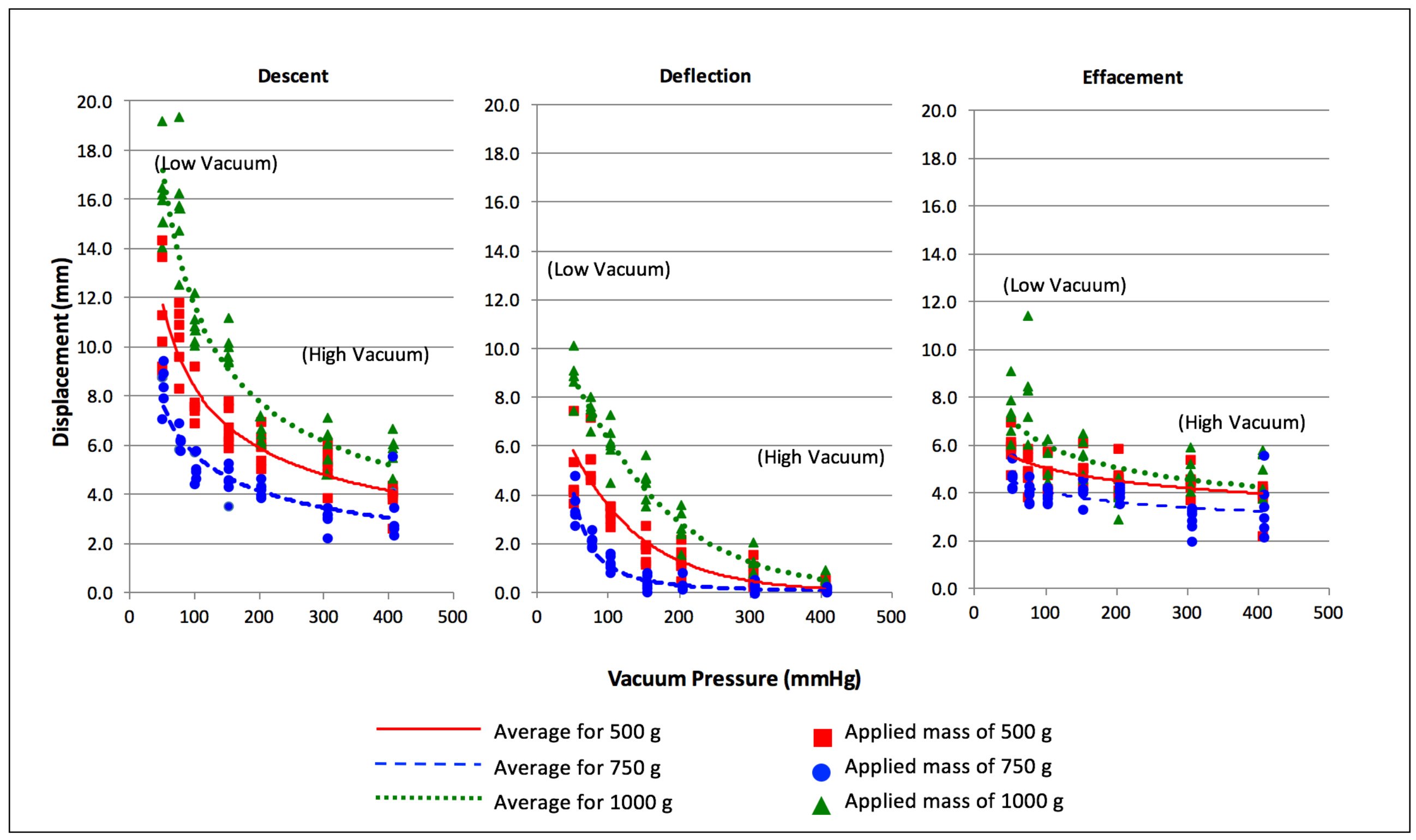

Figure 30: The response of the cervix phantom to labour induction mechanisms 


\subsubsection{Passive Dilation}

The dilation of the cervix balloon as the vacuum pressure was varied from $406.4 \mathrm{mmHg}$ to 50.8 $\mathrm{mmHg}$ is as shown in Figure 31. Overall, it can be observed that greater dilation was observed at lower vacuum pressures, while minimal dilation was observed at vacuum pressures greater than $304.8 \mathrm{mmHg}$. The inner diameter of the cervix balloon, representing the cervical os, deforms in a non-uniform manner as force is applied. This was accounted for by measuring the change in the surface area of the non-uniform polygon shape. The change in diameter (i.e. dilation) was then determined from the surface area, assumed a circular opening.

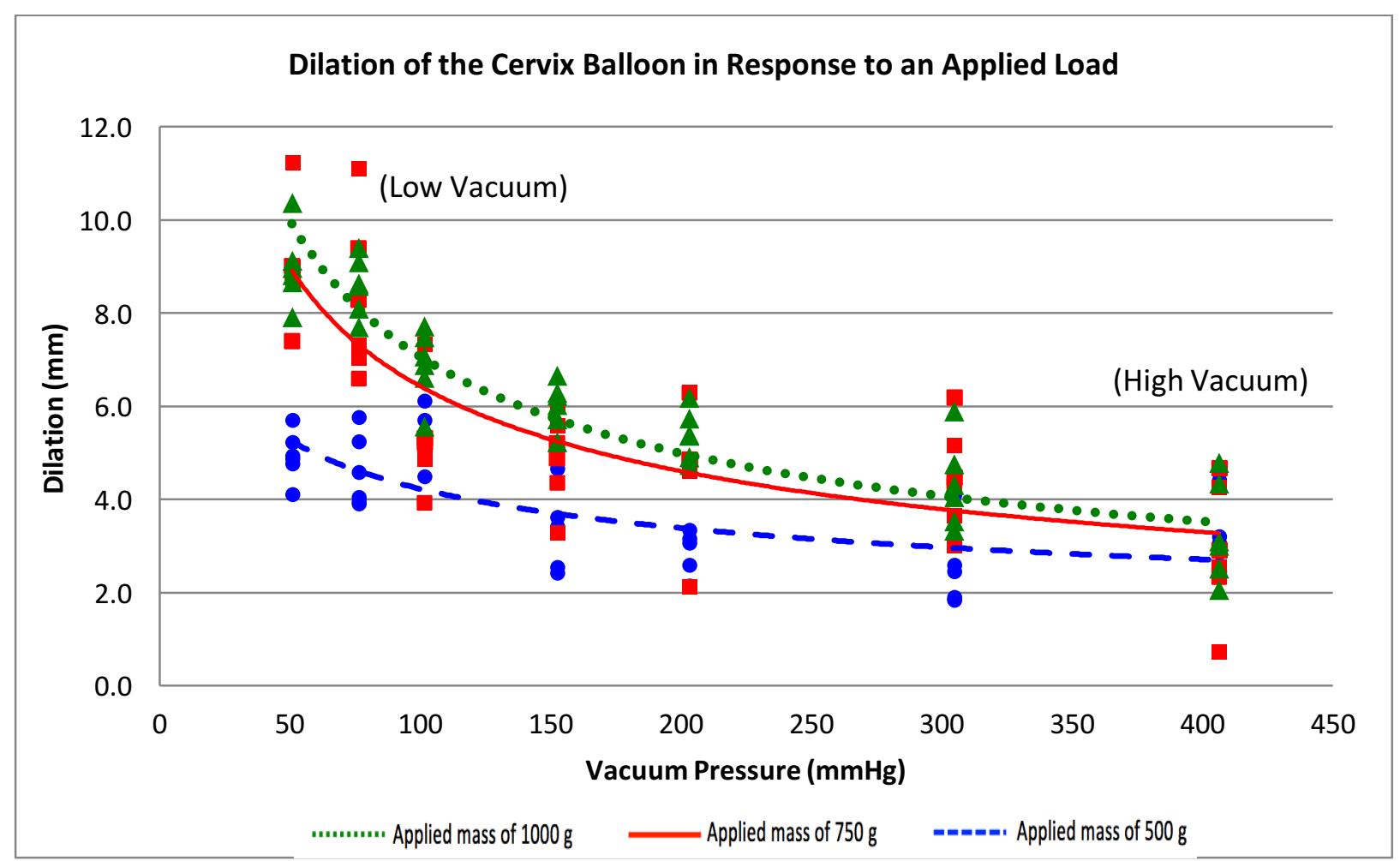

Figure 31: The amount of dilation of the cervix balloon as a function of vacuum pressure applied 


\section{Discussion}

\subsection{Mechanical Properties of Ex Vivo Post-Hysterectomy Tissue}

In the following section, the mechanical properties of the ex vivo post-hysterectomy tissue are discussed. The trends in tissue strength and fatigue properties were analyzed and results were compared to existing literature values. Finally, the significance of mechanical characteristics with respect to the cervix simulation training system was explored.

\subsubsection{Compressive Tissue Strength}

Whole, intact cervix specimens were used in this study to determine the stiffness of non-pregnant cervical tissue. This is in contrast to existing mechanical studies of the cervix, where excised sections of tissue were tested [57]-[59]. By testing whole specimens, the tactile interaction during digital vaginal examinations was quantified. Experimental results indicated that the true stress of the non-pregnant cervix ranges between 100 to $300 \mathrm{kPa}$ when a strain of 0.5 was applied. These findings can be incorporated into a cervix simulation training model, serving as the upper boundary of stiffness at the beginning of labour. Future studies on pregnant tissue from rare placenta accreta hysterectomy cases will be required in order to determine the lower boundary of stiffness following cervical ripening.

While similar trends were observed for all specimens, individual cervices had notably different mechanical strength values. This is expected, as samples were obtained from patients over a wide age range with different pregnancy histories. Age and pregnancy history are two documented factors that influence cervix strength. Younger patients have stiffer cervices than older patients [58]. Similarly, patients who have had no previous vaginal deliveries have stiffer cervical tissue than patients who have had at least one vaginal delivery [57], [58]. These findings suggests that in clinical practice, it is critical for practitioners to identify a range of stiffness that corresponds to each stage of labour. Hence, the stiffness of the training model at the beginning of labour should not be a single value. Rather, a range of compliance values need to be incorporated into the simulation training system such that students can be familiarized with the range of tactile responses corresponding to each stage of labour. Specifically, a range of applied vacuum pressure values 
would correspond to each stage of labour such that students would correspond a range of stiffness values (analogous to different patients) with each stage.

A significant difference in strength was also observed when the cervical tissue was tested at different stress points. This difference can be attributed to the toroid shape of the cervix, as the entire cervix is composed of homogenous fibrous connective tissue [13]. These results are consistent with stress analysis conducted on toroidal shells. As shown in Figure 32, Purdel and Stere [63] demonstrated that the maximum principle stress of a torus was highest in the inner region and smallest in the outer region using Finite Element Analysis. As the range of strength along the cervix is an inherent geometric property, it is expected that similar results will be obtained in the simulation training model as long as the original geometric shape is preserved.

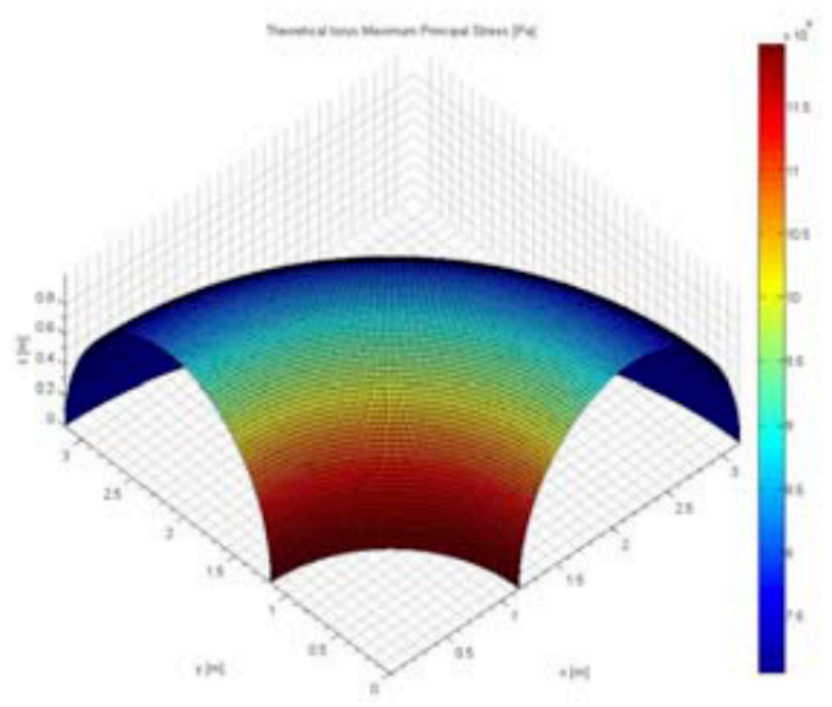

Figure 32: Maximum principle stress of a theoretical torus is highest in the inner region and smallest in the outer region, from [63].

\subsubsection{Fatigue of Tissue}

Repeated applied loading to the cervical tissue was found to have a weakening effect on the tissue at higher strains. This may be due to a disruption in the fibrous matrix in the cervical tissue caused by loading. During continuous trials, the matrix did not have time to fully recover from the loads. At lower strains, minimal disruption was caused and the fibrous matrix could reform before the subsequent loading. The observed fatigue is consistent with compressive strength tests conducted by Yao et al. [58]. As shown in Figure 33, a time-dependent force response was observed when 
indentations were conducted on cervical tissue slices. This force-relaxation phenomenon was accounted for by allowing a 1 minute recovery time between trials in their experimental design [58].

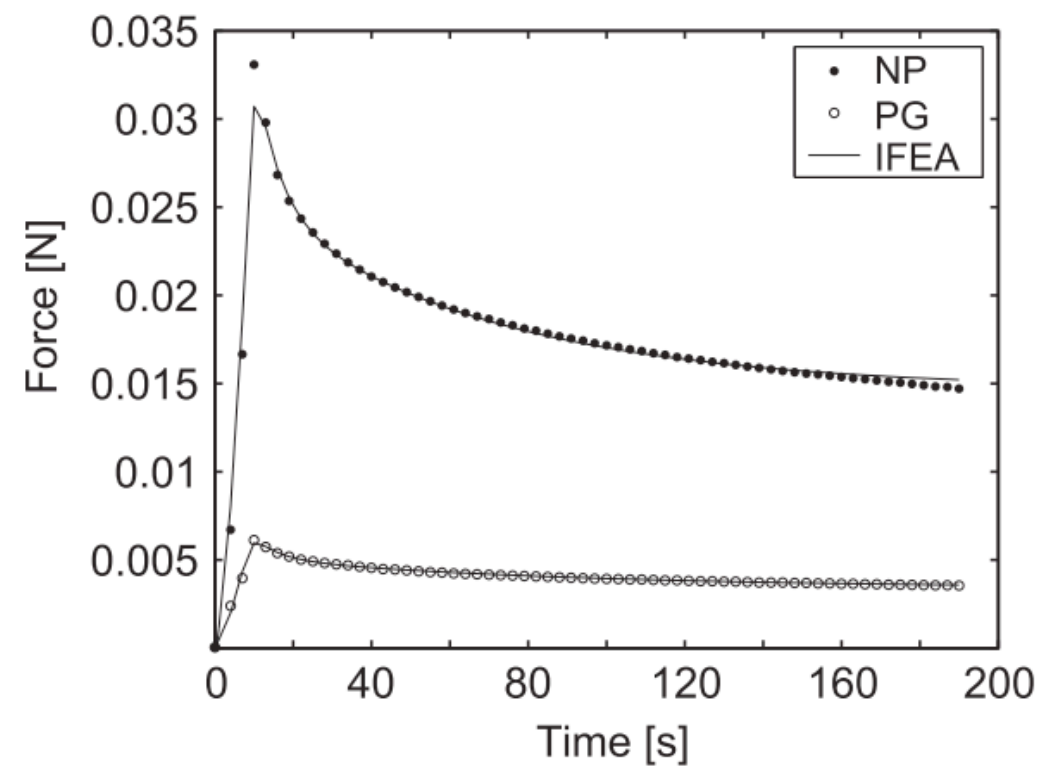

Figure 33: Force relaxation response of non-pregnant (NP) and pregnant (PG) human cervical tissues shown with dotted and circled points, respectively. Two solid lines show the inverse finite element analysis (IFEA) curves for both cases. From [58].

During normal labour, digital vaginal examinations are conducted every four hours, where the stiffness of the cervix is determined tactilely each time [2]. Hence, the cervical tissue has significant time to recover between each examination and the fatigue properties of the cervix will not be observed clinically. Hence, fatigue will not be considered in the design of the simulation training model.

\subsection{The Cervix Phantom Prototype}

The efficacy of the cervix phantom prototype to simulate latent labour was assessed based on its ability to: (1) mimic the compliance of actual cervical tissue, (2) soften using particle jamming to simulate cervical ripening, (3) be integrated into existing simulators as a training tool and (4) dilate and efface in response to labour induction. The following section details the degree to which the phantom fulfills these criteria. Recommendations to improve the phantom prototype are also discussed. 


\subsubsection{Comparison with Ex Vivo Post-Hysterectomy Tissue and Existing Models}

Figure 34 compares the stress-strain curve of the cervix phantom at its softest setting (i.e. $0 \mathrm{kPa}$ ) to that of actual cervical tissue and the softest setting (i.e. $20 \mathrm{kPa}$ ) of the 3D printed model presented in Jung Kim's MASc thesis [43]. The stress-strain curve of the cervix phantom was measured at $0 \mathrm{kPa}$ through 10 compressive test trials. The cervical tissue stress-strain curve was averaged from compressive tests conducted on ten post-hysterectomy cervical specimens. The stress-strain curve from Kim's model was adapted from Kim's MASc thesis [43]. While the applied strain differs in Kim's experiments, it is evident that Kim's 3D printed model is much stiffer than actual cervical tissue. Specifically, Kim reported that the model has a maximum stress ten orders of magnitude higher than that of human cervical tissue [43]. The high stiffness in Kim's model was due to manufacturing constraints imposed by the 3D printer and available filaments. NinjaFlex elastomeric filament was selected due to its durability and reliability [43]. The resulting model was anatomically accurate, but much stiffer than cervical tissue. In the present cervix phantom, greater softness was achieved by using latex in place of the elastomer. As an anatomically accurate latex model could not be produced given available manufacturing tools, a commercial toroid balloon was ultimately selected.

The stress-strain characteristics of the present cervix phantom at $0 \mathrm{kPa}$ is comparable to that of the non-pregnant human cervical tissue, indicating that the phantom is successful in simulating the compliance of the cervix prior to softening. However, at higher vacuum pressures, the cervix phantom is stiffer than the non-pregnant cervix tissue. Therefore, the present cervix phantom range of compliance needs to be lower such that cervical ripening can be accurately simulated. This can be achieved by varying the amount of coffee grounds in the balloon model. If the amount of grounds were decreased, there would be greater interstitial space between coffee grounds at all applied vacuum pressures and the model would become softer overall. Alternatively, positive pressures can also be explored to increase interstitial space and soften the model. 


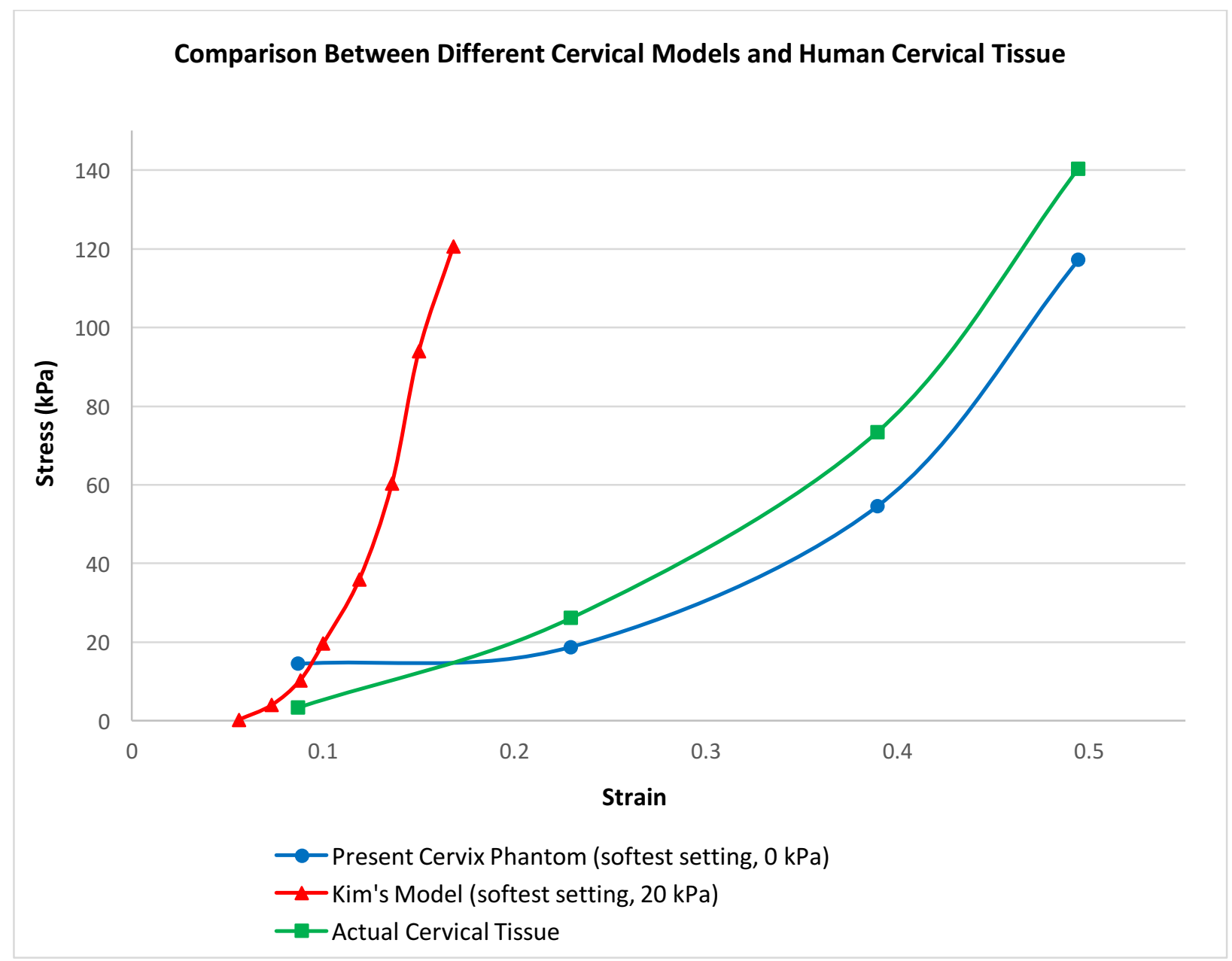

Figure 34: The compressive strength of the cervix phantom at its softest setting $(0 \mathrm{kPa})$ is comparable to the strength of actual cervical tissue, while the softest setting of Kim's model $(20 \mathrm{kPa})$ was much stiffer, Kim's data adapted from [43].

\subsubsection{Simulating Latent Labour}

Using particle jamming theory [51], the cervix phantom prototype achieved dynamic compliance to effectively simulate the various stages of latent labour. Table 4 shows the vacuum pressure setting corresponding to different stages of latent labour and the consistency portion of the Bishop Score. Prior to the beginning of labour, the main role of the cervix is to maintain the fetus within the uterus and withstand forces from the weight of the fetus, amniotic sac and pressures from the uterine wall [18] (i.e. consistency score of 0 ). This stage was simulated in our cervix phantom by deflating the phantom through applying a vacuum pressure of $406.4 \mathrm{mmHg}$. The cervix phantom was a rigid body in this setting, exhibiting negligible effacement or dilation in response to tractive forces. At the onset of labour, the cervix begins to soften such that it can be pulled open by uterine 
contractions to allow the newborn's head to pass through. This process, known as cervical ripening, is a result of matrix remodeling and a reorganization of the collagen fibrillar network [13]. Cervical ripening was simulated by decreasing the vacuum pressure in the cervix phantom from $406.4 \mathrm{mmHg}$ to $0 \mathrm{mmHg}$. As vacuum pressure decreased, interstitial space between individual coffee grounds was increased such that the phantom gradually softened. At around 83.3 $\mathrm{mmHg}$, interstitial space increases such that the compliance of the phantom transitions from hard to soft (i.e. consistency score of 1). At pressures lower than $83.3 \mathrm{mmHg}$, the phantom feels soft to the touch (i.e. consistency score of 2).

Table 4: The Vacuum Pressure Corresponding to Different Stages of Latent Labour

\begin{tabular}{|c|c|c|c|}
\hline $\begin{array}{c}\text { Consistency Portion } \\
\text { of Bishop Score }\end{array}$ & 0 & 1 & 2 \\
\hline Compliance & Firm & Medium & Soft \\
\hline $\begin{array}{c}\text { Vacuum Pressure in } \\
\text { the Phantom }\end{array}$ & $\begin{array}{c}123.3 \mathrm{mmHg}-406.4 \\
\mathrm{mmHg}\end{array}$ & $43.3-123.3 \mathrm{mmHg}$ & $0-43.3 \mathrm{mmHg}$ \\
\hline
\end{tabular}

\subsubsection{Cervix Phantom in Simulation Training}

Tactile psychophysics experimental results confirm the potential for the cervix phantom to be used as a simulation training tool to help students familiarize with the stiffness of the cervix during various labour stages. All participants detected a distinguishable difference in the stiffness of the cervix phantom as vacuum pressure was decreased. This was quantified in the threshold experiment, where a vacuum pressure of $83.3 \mathrm{mmHg}$ corresponded to the cervix phantom transitioning from "hard" to "soft". Moreover, results were consistent among all participants and repeatable over several trials. Hardness and softness specificities, as defined by the threshold of $83.3 \mathrm{mmHg}$, were $91.4 \%$ and $86.7 \%$, respectively. Consistency and repeatability in tactile perception of our participants indicate the efficacy of using simulation training models. It is expected that with training, students interacting with the cervix phantom will learn to correspond the tactile feedback to the different labour stages. Ultimately, adaptation of a realistic cervical model is expected to lead to greater diagnostic accuracy of labour stages during digital vaginal examinations. 


\subsubsection{Responding to Labour Induction}

The cervix phantom demonstrated passive effacement and dilation in response to a tractive force, as the vacuum pressure was decreased from $406.4 \mathrm{mmHg}$ to $0 \mathrm{mmHg}$ (i.e. slowly re-inflated to atmospheric pressure from a high vacuum). This indicates that the phantom, like the actual cervix, softens significantly such that it can be pulled opened for the newborn's head to pass through. However, the amount of effacement and dilation in our phantom was much less than that of the actual cervix during latent labour. A maximum effacement of $0.7 \mathrm{~cm}$ and dilation of $1 \mathrm{~cm}$ was achieved in the cervix phantom, while dilation of $3 \mathrm{~cm}$ and effacement of $80 \%$ (i.e. shortening by $\sim 1.6 \mathrm{~cm}$ ) is observed in the actual cervix during latent labour [64]. Therefore, a second-level of control is required to provide consistent dilation and effacement behavior that can be varied depending on the simulation scenario at hand. One recommended approach to achieving this would be through the addition of actuators to pull on the outer walls of the cervix model, as previously demonstrated by Smith et al. [65] and shown in Figure 35.

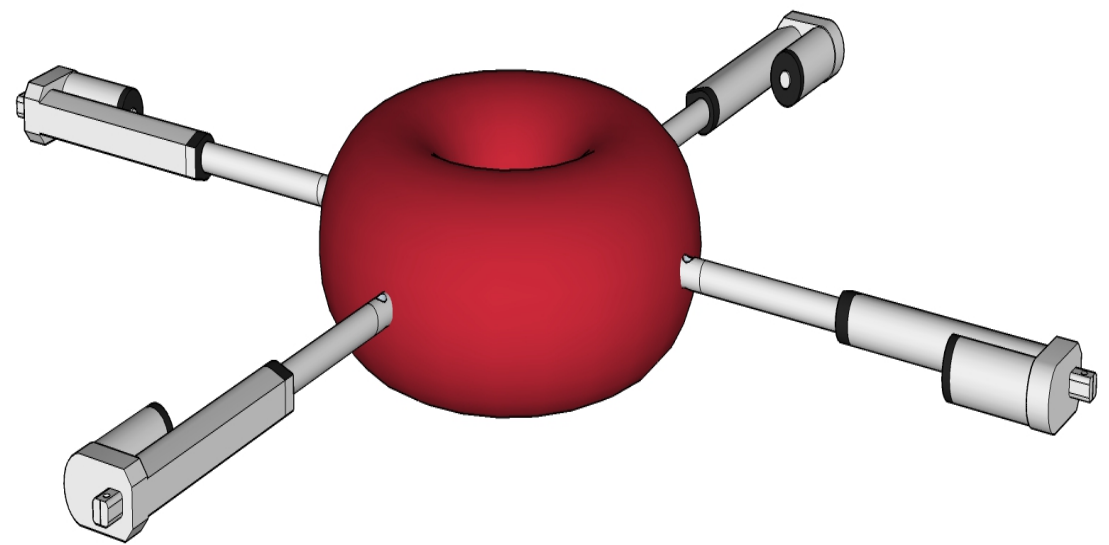

Figure 35: Actuators can be added to the outer walls of the cervix model.

\subsubsection{Anatomical Accuracy}

The cervix phantom features a toroid shape representative of actual cervical tissue. However, as the present phantom utilizes a commercial latex balloon, it remains larger with a diameter of 40.64 $\mathrm{cm}$ (versus $3 \mathrm{~cm}$ diameter of a real cervix). Anatomical accuracy can be achieved in future models using the 3D scanned data of the post-hysterectomy tissue and new technologies that allow for printing with silicone and latex materials. Exploration of the Universal Paste Extruder by Structur3d Printing is recommended [66]. This extruder can be added to existing 3D printers, and can dispense soft materials, including silicone, using a syringe cartridge system [66]. 


\subsection{Significance of Results}

The work presented in this thesis paper serves as a foundation towards the design of a new dynamic human birth simulation training system. Table 4 summarizes the pertinent characteristics and behavior of the cervix during latent labour, and compares the behavior of actual cervical tissue with the preliminary phantom prototype. The phantom prototype was successful in simulating the compliance of the cervix at the onset of labour and demonstrate similar softening characteristics, as indicated by the checkmarks in Table 4. While certain discrepancies remain, as indicated by the crosses in Table 4, the phantom prototype provides a baseline for future simulation training models. Particularly, the use of particle jamming to simulate cervical ripening is extremely effective. In fact, the phantom is actually capable of more softening than required to simulate cervical ripening during latent labour. The maximum stress of the cervix phantom decreased by 22 times (i.e. from $419 \mathrm{kPa}$ to $18.8 \mathrm{kPa}$ ) when a strain of 0.23 was applied and the vacuum pressure was decreased from $406.4 \mathrm{mmHg}$ (i.e. high vacuum) to $0 \mathrm{mmHg}$ (i.e. atmospheric pressure). In contrast, during latent labour, the cervix only softens by approximately 7 times according to compression studies done on pregnant and non-pregnant excised cervical tissue [57].

Table 5: Comparison between Cervix Tissue and Phantom Prototype

\begin{tabular}{|c|c|c|c|}
\hline \multirow[b]{2}{*}{ Characteristic } & \multicolumn{2}{|l|}{ Cervix Tissue } & \multirow[b]{2}{*}{ Phantom Prototype } \\
\hline & From Literature & $\begin{array}{l}\text { Experimental } \\
\text { Results }\end{array}$ & \\
\hline $\begin{array}{l}\text { Stiffness at } \\
\text { onset of labour }\end{array}$ & - & $\begin{array}{l}\checkmark \text { Maximum stress } \\
\text { of } 75-250 \mathrm{kPa} \\
\checkmark \text { Rigid structure } \\
\end{array}$ & $\begin{array}{l}\checkmark \text { Maximum stress of } 117 \mathrm{kPa} \\
\text { (at atmospheric pressure) } \\
\checkmark \text { Rigid structure }\end{array}$ \\
\hline Softening & $\begin{array}{l}\checkmark \text { Cervix becomes } \\
7 \text { times softer }\end{array}$ & - & $\begin{array}{l}\checkmark \text { Phantom became } 22 \text { times } \\
\text { softer }\end{array}$ \\
\hline Effacement & $\begin{array}{l}\checkmark \text { Cervix effaces } \\
80 \%^{*}\end{array}$ & - & $\times$ Phantom effaced $35 \%$ \\
\hline Dilation & $\begin{array}{l}\checkmark \text { Cervix dilates } 3 \\
\mathrm{~cm}^{*}\end{array}$ & 一 & $\times$ Phantom dilates $1 \mathrm{~cm}$ \\
\hline Shape & $\begin{array}{l}\checkmark \text { Toroid, } 3 \mathrm{~cm} \\
\text { outer diameter }\end{array}$ & - & $\begin{array}{l}\times \text { Toroid, } 10 \mathrm{~cm} \text { outer } \\
\text { diameter }\end{array}$ \\
\hline
\end{tabular}

*Adapted from Myers et al. [57] 


\section{Conclusions}

The present work has addressed shortcomings of existing human birth simulation systems, which lack a dynamic cervix to simulate cervical ripening during latent labour. The following are the major contributions introduced in this thesis:

- Mechanical characteristics of the cervix, including the stress-strain characteristics, fatigue properties and the strength of different stress points, were determined through testing of post-hysterectomy cervical specimens. The cervix exhibited the greatest strength proximal to the cervical os (i.e. 4-325 kPa), moderate strength at the center (i.e. 3-275 kPa) and lowest strength at the distal stress point (i.e. 3-180 kPa).

- Particle jamming theory was used to create a cervix phantom with dynamic compliance in response to varying vacuum pressure. When vacuum pressure decreased from the maximum applied vacuum of $406.4 \mathrm{mmHg}$ to atmospheric pressure, the maximum stress of the cervix phantom decreased by 22 times (i.e. from $419 \mathrm{kPa}$ to $18.8 \mathrm{kPa}$ ) at a strain of 0.23 .

- The response of the cervix phantom to labour induction was characterized using optical sensors and the MATLAB R2015 Image Processing Toolbox. The passive effacement and dilation of the phantom can be controlled through varying vacuum pressure. Maximum effacement of $35 \%$ and dilation of $1 \mathrm{~cm}$ was observed.

In conclusion, findings presented in this paper yield a deeper understanding of cervix characteristics and establish groundwork for the design of a dynamic human birth simulation training system. The established workflow and tissue testing platform can be used in future studies to further explore cervical properties. Specifically, future samples of pregnant tissue obtained from rare placenta accreta hysterectomy cases could be tested using the presented testing platform. Moreover, future mechanical studies can include patient information such as pregnancy history such that correlations in tissue strength and patient-related factors can be determined. With regards to the cervix phantom, greater softening can be accomplished through exploration of optimal amount of granular material fill and the effects of applying positive pressures. Tactile psychophysics tests from expert clinicians can confirm the efficacy of such models. Psychophysics results of untrained volunteers, midwives and obstetricians can 
be compared. Additionally, while investigations with particle jamming theory demonstrate the feasibility for a dynamically compliant cervix phantom, current findings will need to be retrofitted to an anatomical accurate non-porous flexible toroid. Using the NextEngine 3D Scanner Ultra HD, curvatures and textures of the post-hysterectomy specimens were captured and replicated in an anatomically accurate 3D printed model. The 3D-scanned specimens can be used to fabricate anatomically accurate and soft models using appropriate technological tools in future studies to replace the existing commercial toroid. The work presented in Kim's thesis relating to printing soft models can be continued to fabricate a watertight $3 \mathrm{D}$ printed latex or silicone model. Finally, a second-level of control is required to provide consistent dilation and effacement behavior. The use of actuators on the outer walls of the model is recommended to accomplish this. 


\section{Appendix}

\section{A1 Source Code}

\section{A1.1 Arduino Code for Tissue Indentation}

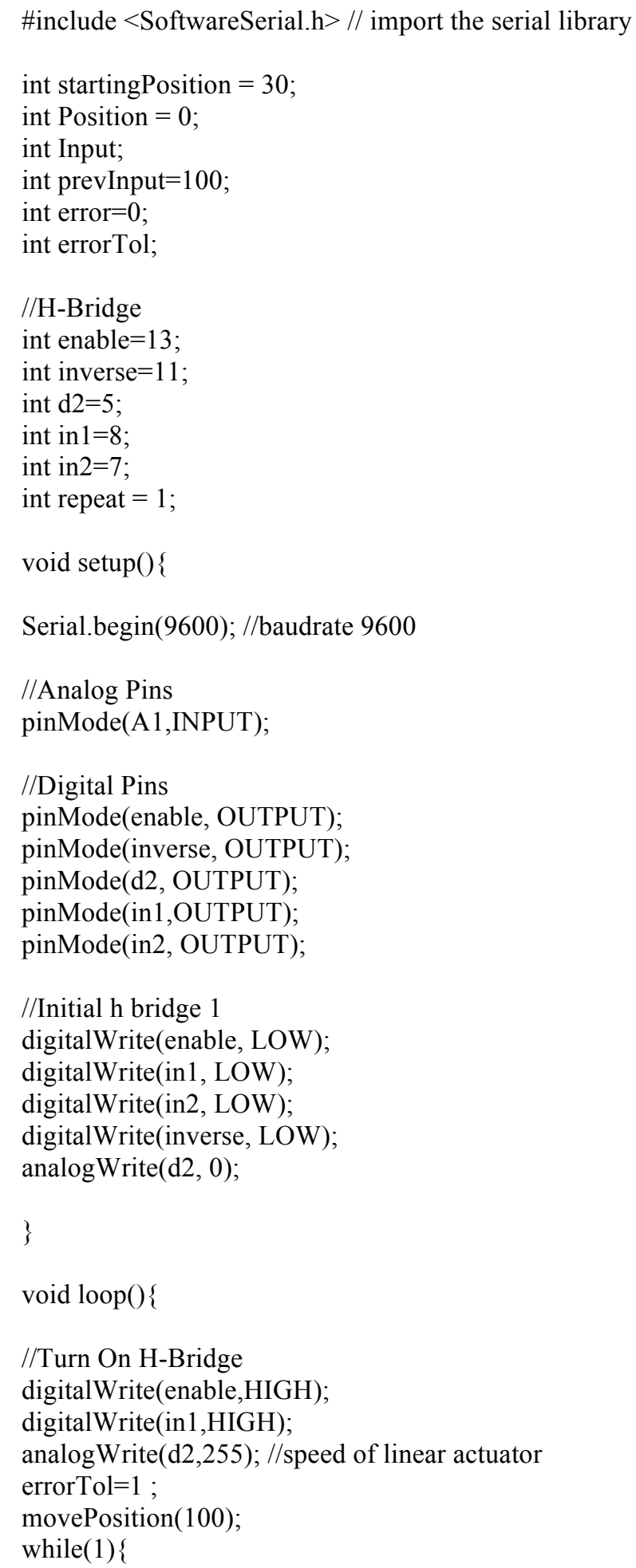




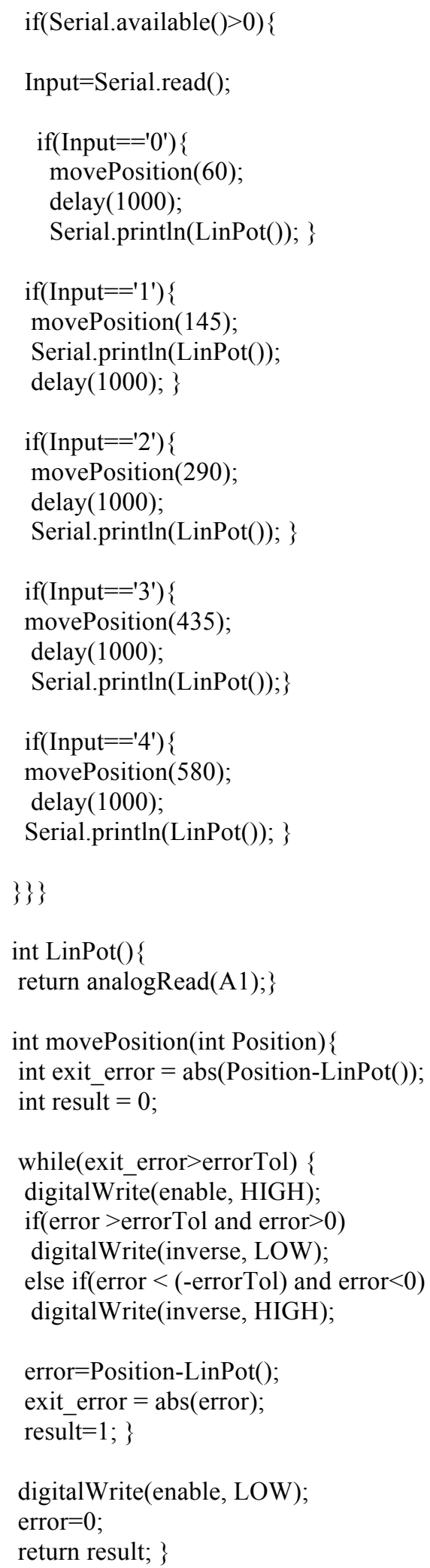




\section{A1.2 MATLAB Code to Calculate Dilation}

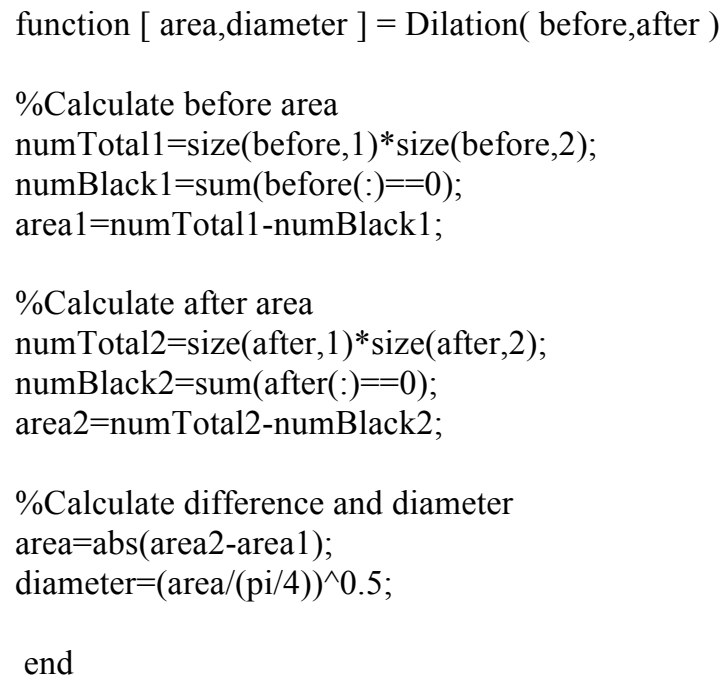

\section{A1.3 Kinect Camera Code for Measuring Effacement}

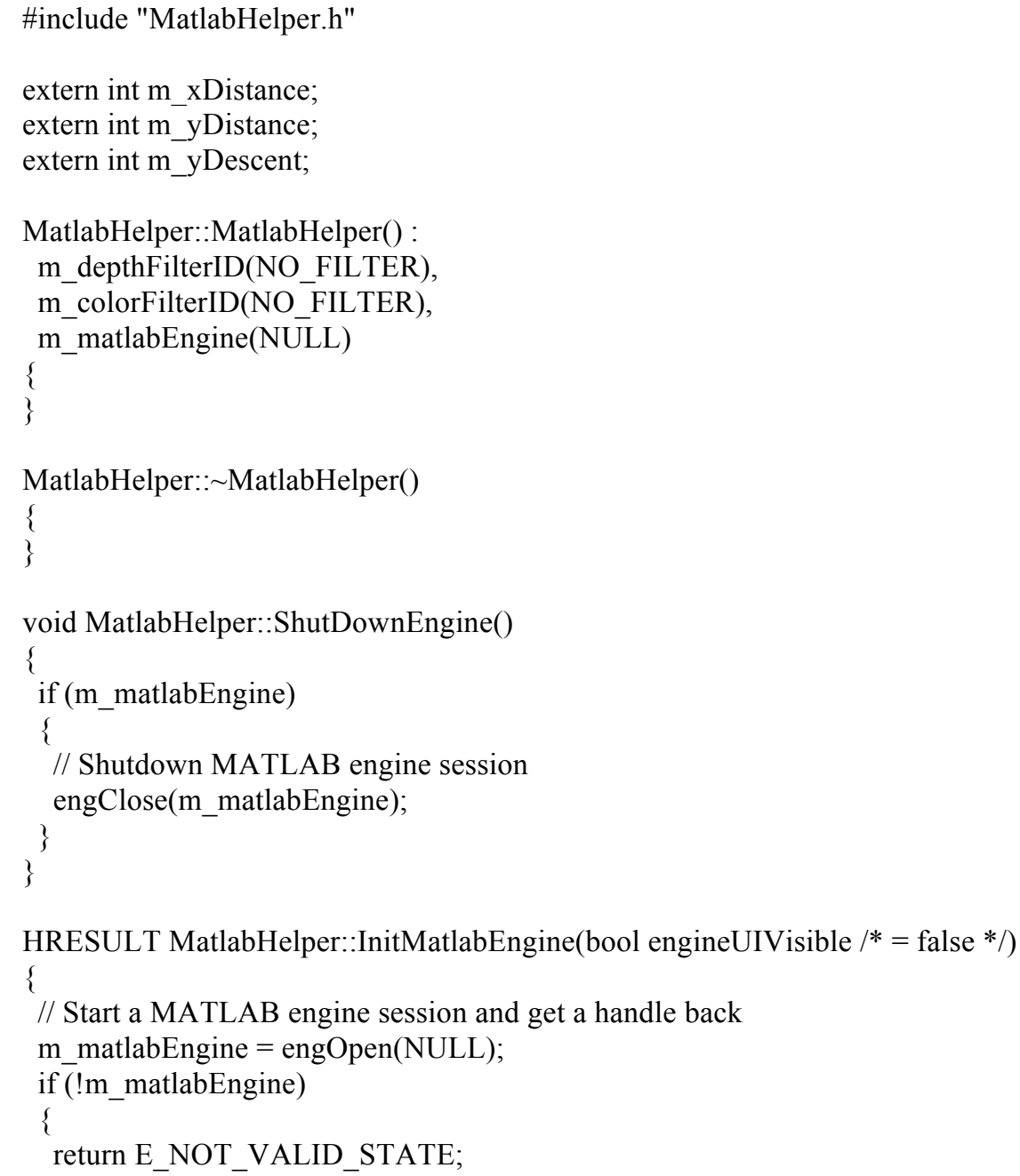




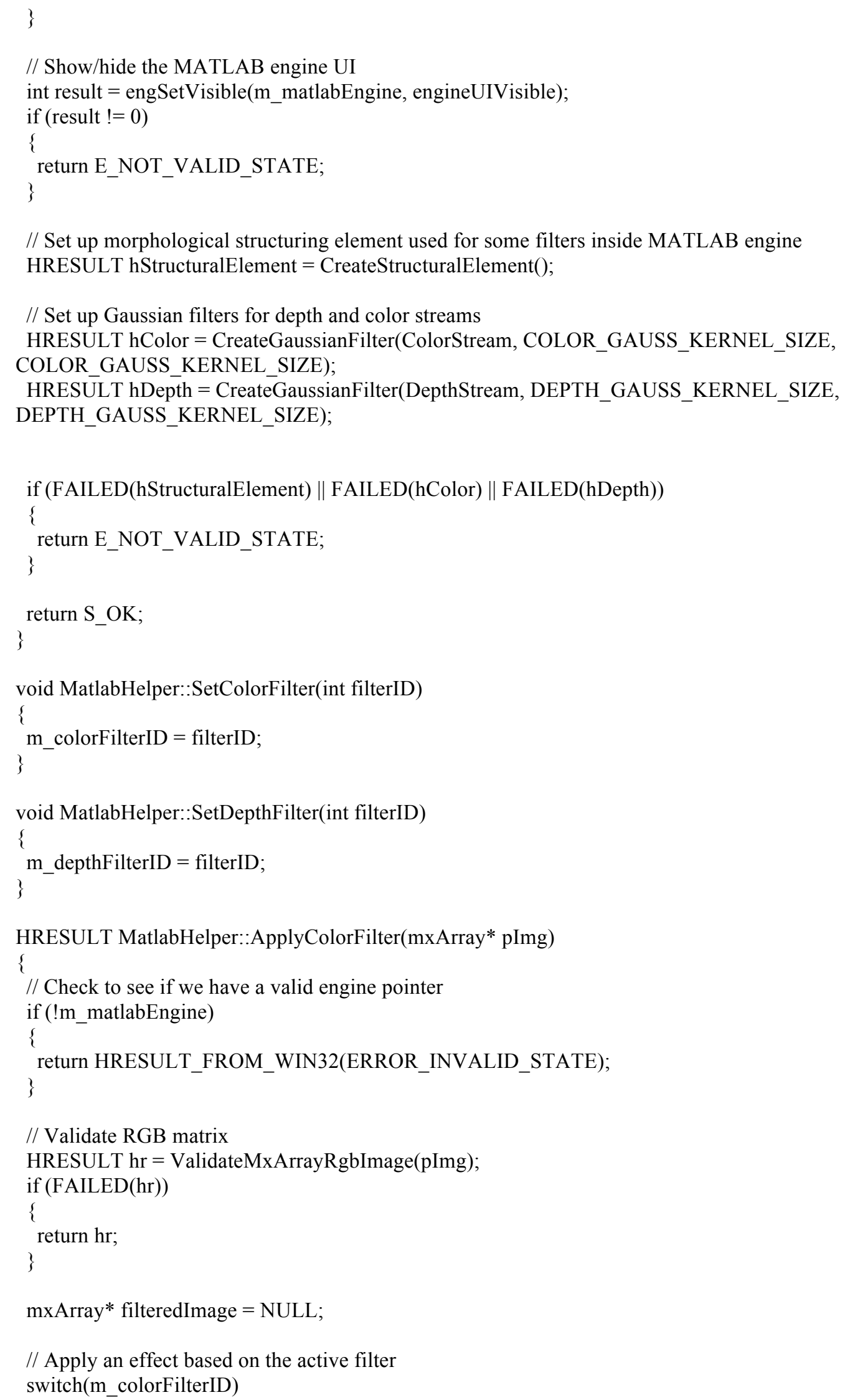




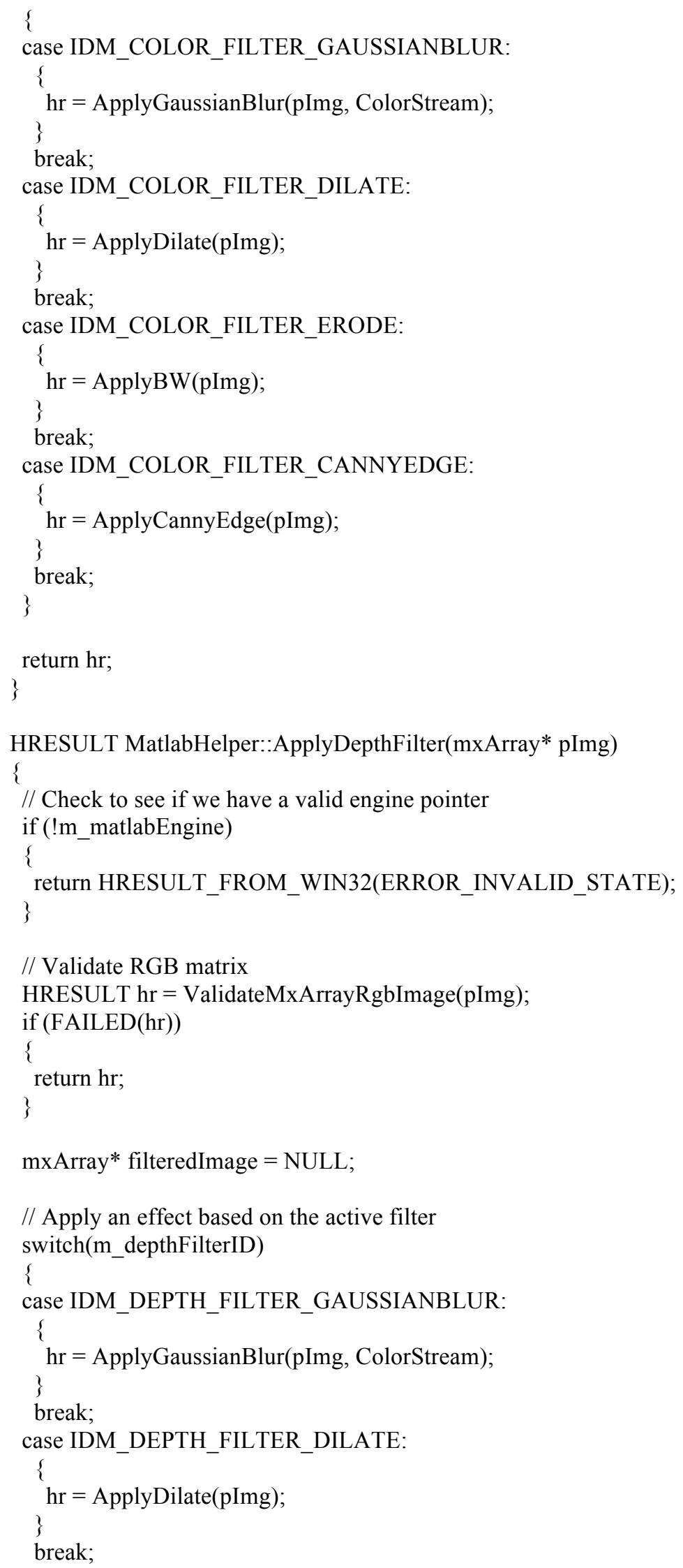




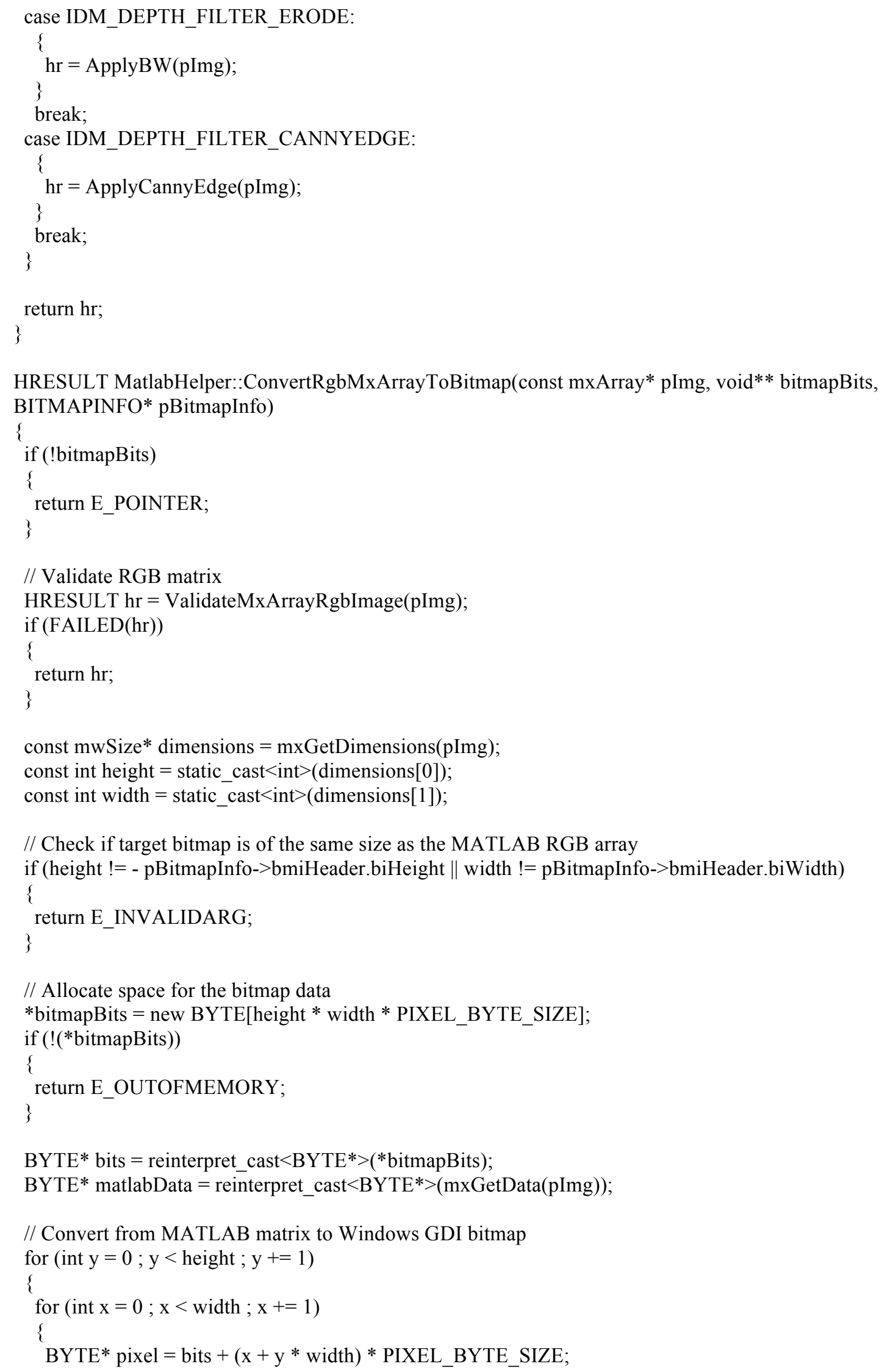




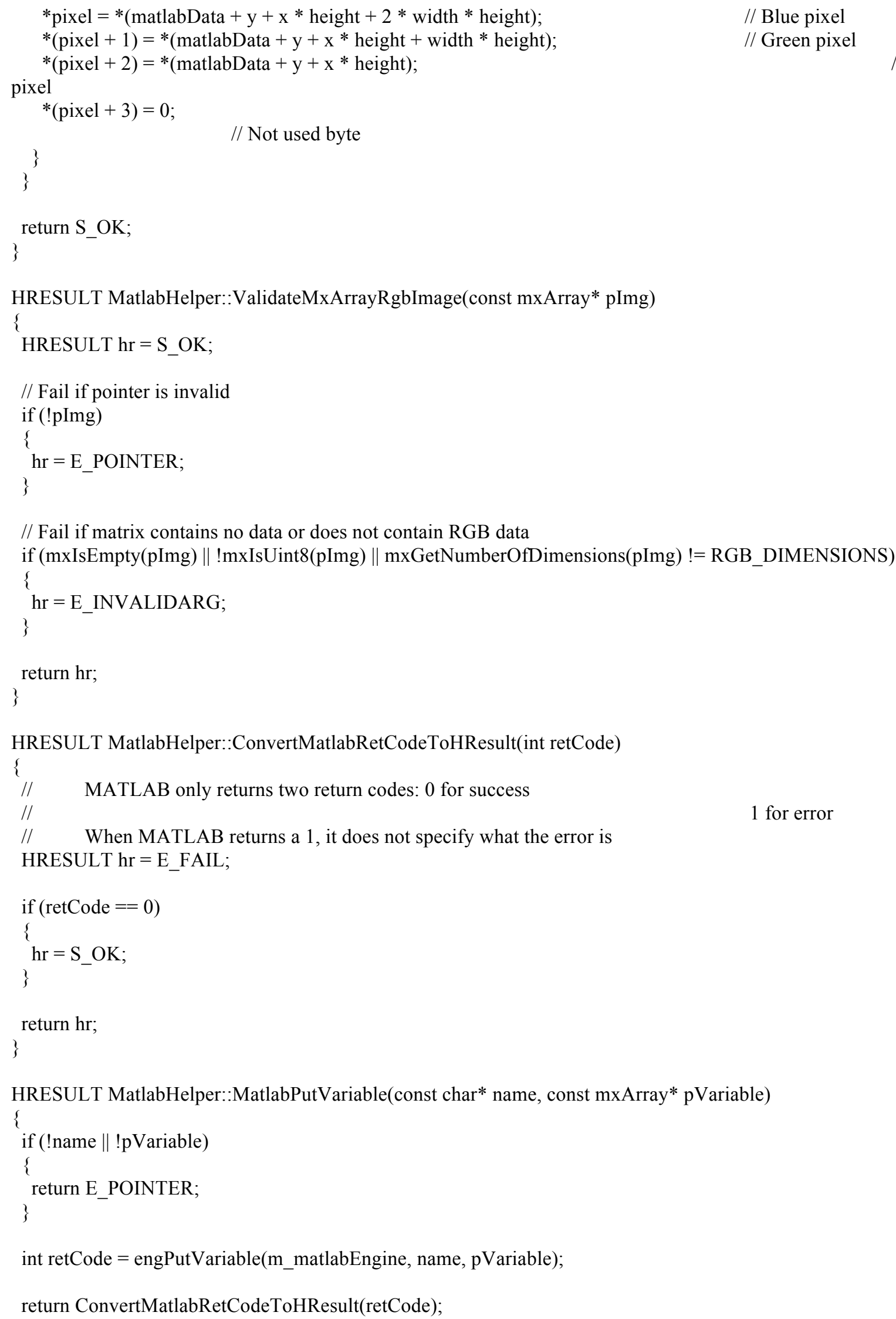


HRESULT MatlabHelper::MatlabGetVariable(const char* name, mxArray** ppVariable)

\{

if (!name $\|$ !ppVariable)

\{

return E_POINTER;

\}

mxArray* $\mathrm{pVar}=$ engGetVariable $\left(\mathrm{m} \_\right.$matlabEngine, name $)$;

if (!pVar) \{

// engGetVaraible only returns NULL if the variable does not exist in the MATLAB environment return E_NOT_SET;

\}

*ppVariable $=$ pVar;

return S_OK;

\}

HRESULT MatlabHelper::MatlabEvalExpr(const char* expr)

\{

if (!expr)

\{

return E_POINTER;

\}

int retCode $=$ engEvalString $\left(\mathrm{m}_{-}\right.$matlabEngine, expr $)$;

return ConvertMatlabRetCodeToHResult(retCode);

\}

HRESULT MatlabHelper::ApplyGaussianBlur(mxArray* pImg, StreamType type)

\{

HRESULT hr;

hr = MatlabPutVariable("img", pImg);

if (FAILED(hr))

\{

return hr;

\}

const char* c_applyColorGaussianFilterExpr = "filtered_img = imfilter(img, color_gauss_filter, 'replicate');"; const char* c_applyDepthGaussianFilterExpr = "filtered_img = imfilter(img, depth_gauss_filter, 'replicate');";

// Pick appropriate Gaussian filter based on image stream

if $($ type $==$ ColorStream)

\{

// Apply the Gaussian blur filter

hr = MatlabEvalExpr(c_applyColorGaussianFilterExpr); \}

else if (type $==$ DepthStream)

\{

// Apply the Gaussian blur filter

$\mathrm{hr}=$ MatlabEvalExpr(c_applyDepthGaussianFilterExpr);

\} 


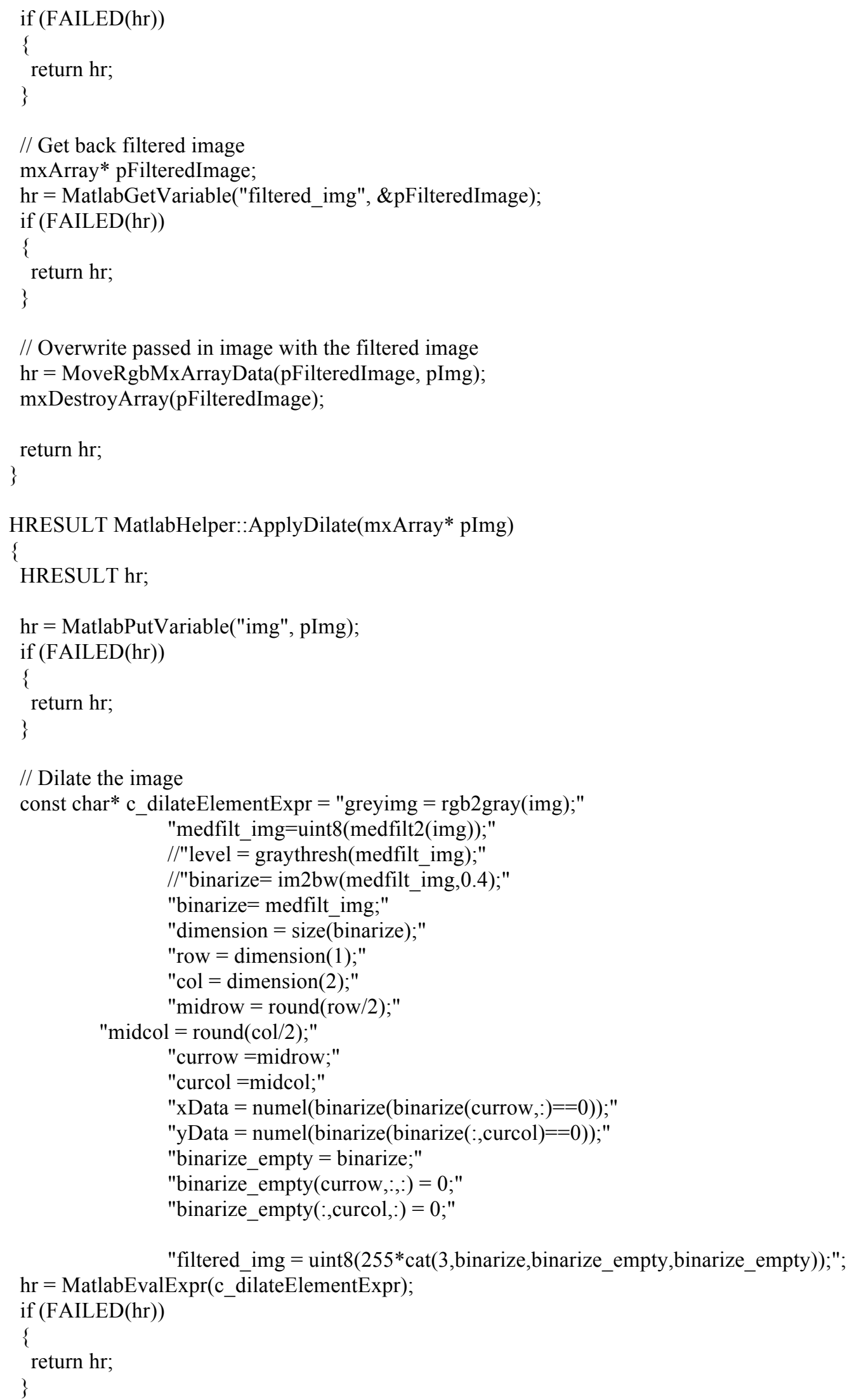




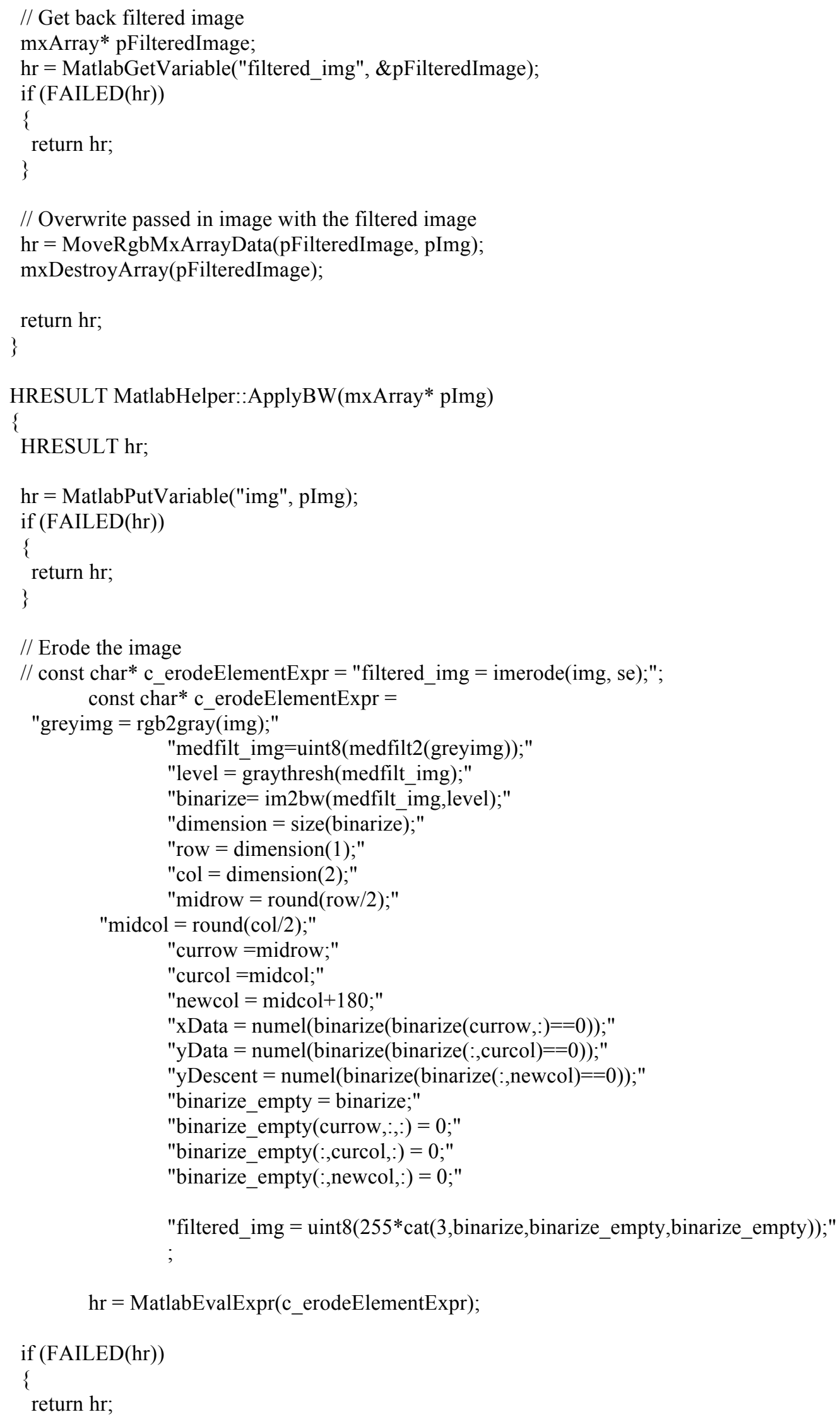




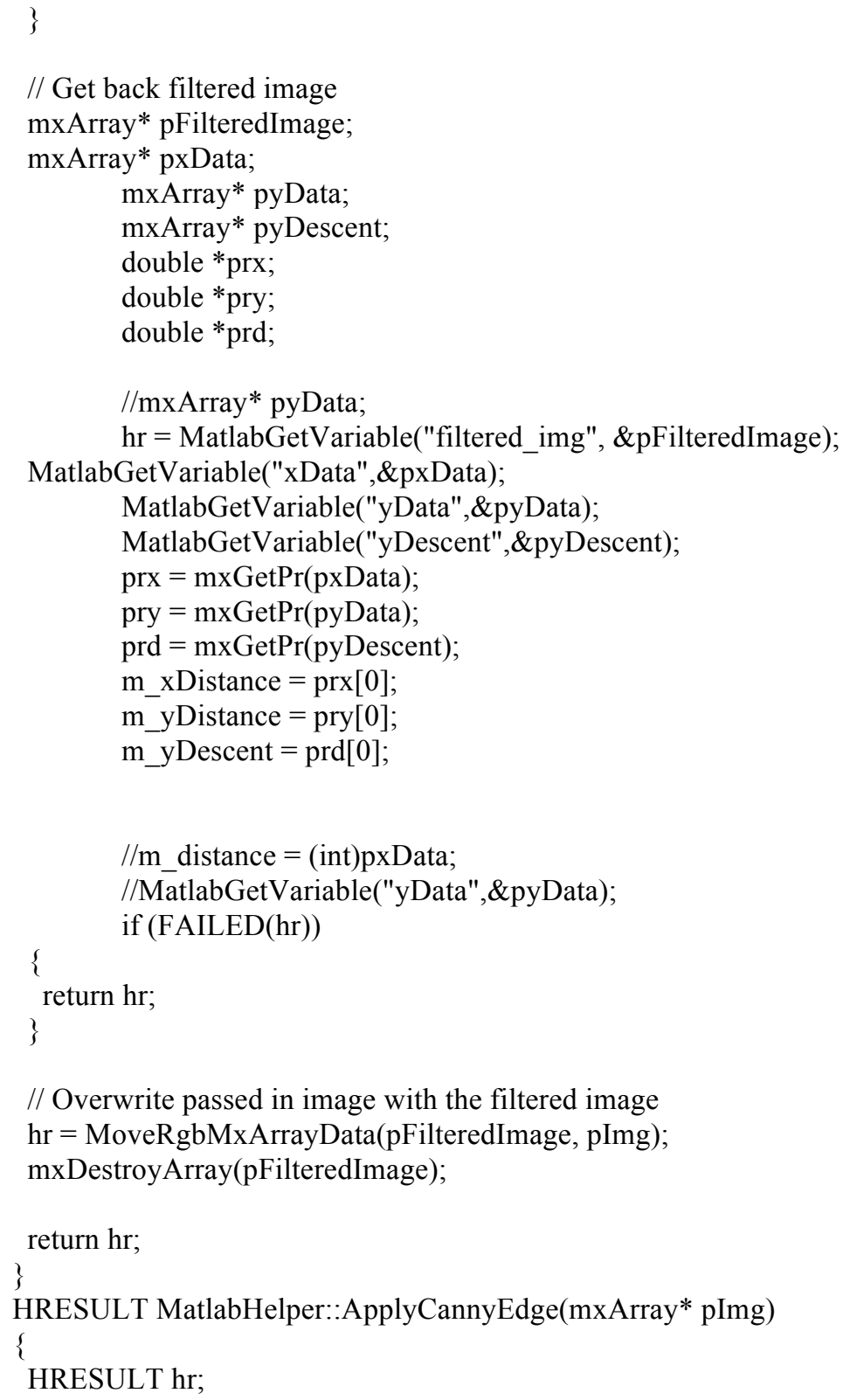

// Convert filtered image from binary into RGB 


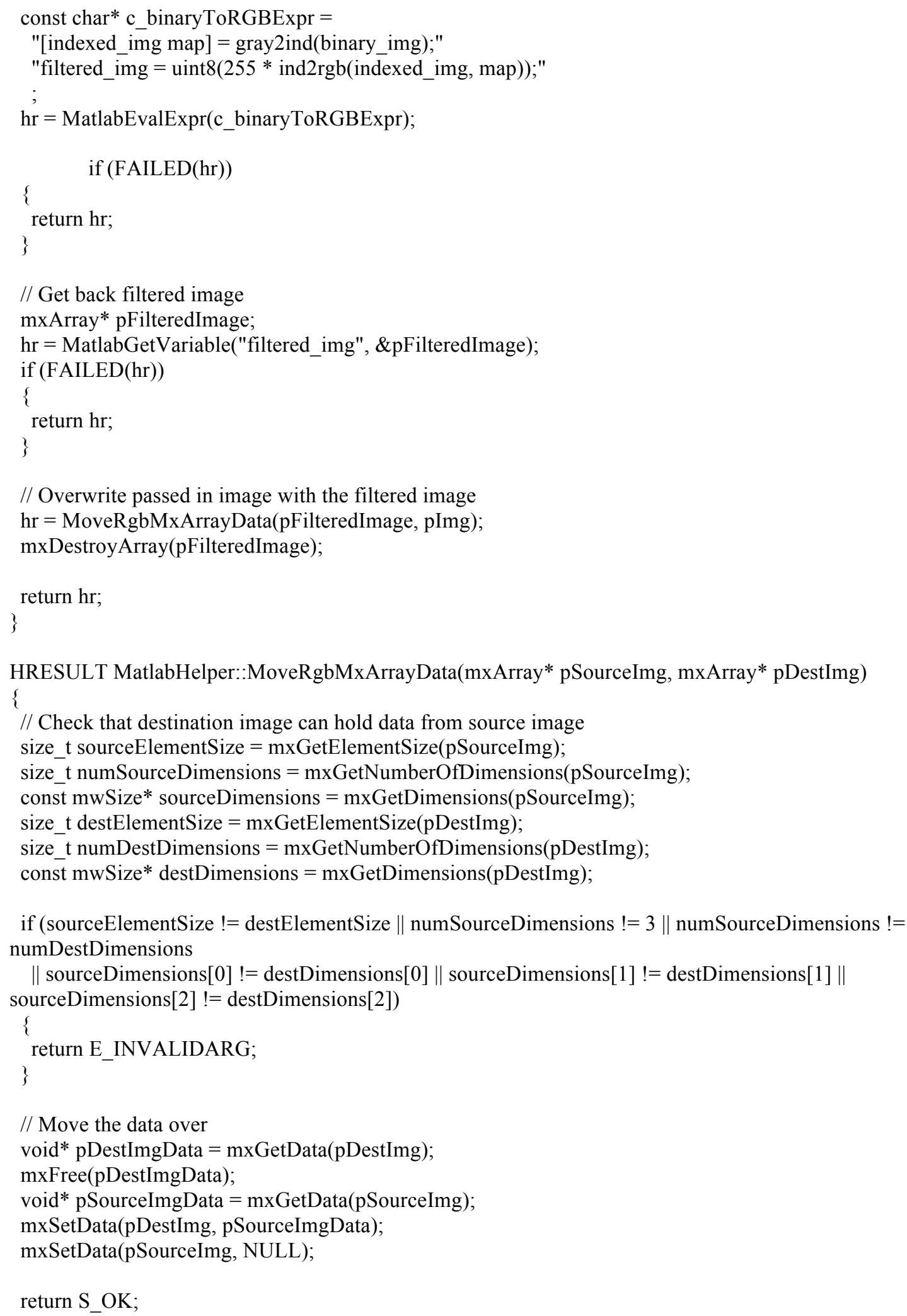




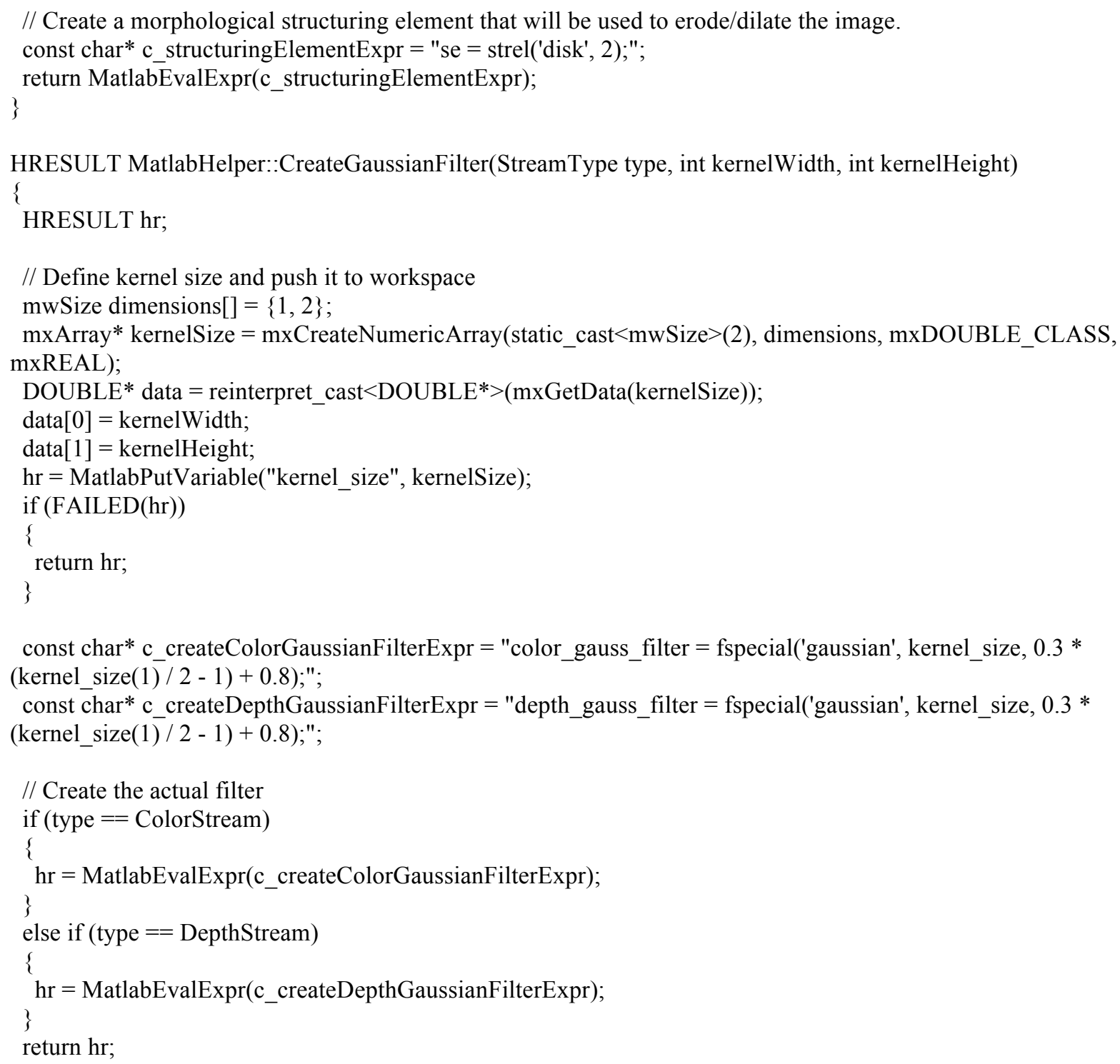




\section{A2 Linear Actuator Calibration}

Table A1: The Calibration of the Firgelli Linear Actuator

\begin{tabular}{|l|l|l|l|l|l|l|l|l|}
\hline \multirow{2}{*}{$\begin{array}{l}\text { Position } \\
\text { No. }\end{array}$} & \multirow{2}{*}{$\begin{array}{l}\text { Digital } \\
\text { Reading }\end{array}$} & \multicolumn{4}{|l|}{ Measured Length $(\mathrm{mm})$} & & \\
\cline { 3 - 9 } & & & 2 & 3 & 4 & 5 & $\begin{array}{l}\text { Average } \\
(\mathrm{mm})\end{array}$ & $\begin{array}{l}\text { Standard } \\
\text { Deviation }\end{array}$ \\
\hline 1 & 380 & 27.86 & 27.15 & 27.46 & 27.43 & 28.00 & 27.58 & 0.35 \\
\hline 2 & 460 & 30.17 & 31.75 & 31.73 & 30.87 & 31.70 & 31.24 & 0.71 \\
\hline 3 & 540 & 35.15 & 34.71 & 35.09 & 35.26 & 35.61 & 35.16 & 0.32 \\
\hline 4 & 620 & 38.90 & 38.67 & 38.53 & 39.04 & 38.93 & 38.81 & 0.21 \\
\hline
\end{tabular}

Linear actuator length was measured with digital calipers to verify the reproducibility of the pre-programmed indentation displacements.

\section{A3 Post-Hysterectomy Cervix 3D Scans}

Table A2: List of Anonymized Hysterectomy Patients

\begin{tabular}{|r|l|r|}
\hline ID & $\begin{array}{l}\text { Date of } \\
\text { Surgery }\end{array}$ & Age \\
\hline 1 & $11 / 23 / 2015$ & 45 \\
\hline 2 & $12 / 8 / 2015$ & 51 \\
\hline 3 & $12 / 16 / 2015$ & 47 \\
\hline 4 & $12 / 16 / 2015$ & 50 \\
\hline 5 & $2 / 9 / 2016$ & 33 \\
\hline 6 & $2 / 19 / 2016$ & 48 \\
\hline 7 & $2 / 19 / 2016$ & 48 \\
\hline 8 & $2 / 26 / 2016$ & 47 \\
\hline 9 & $3 / 22 / 2016$ & 45 \\
\hline 10 & $4 / 20 / 2016$ & 31 \\
\hline
\end{tabular}




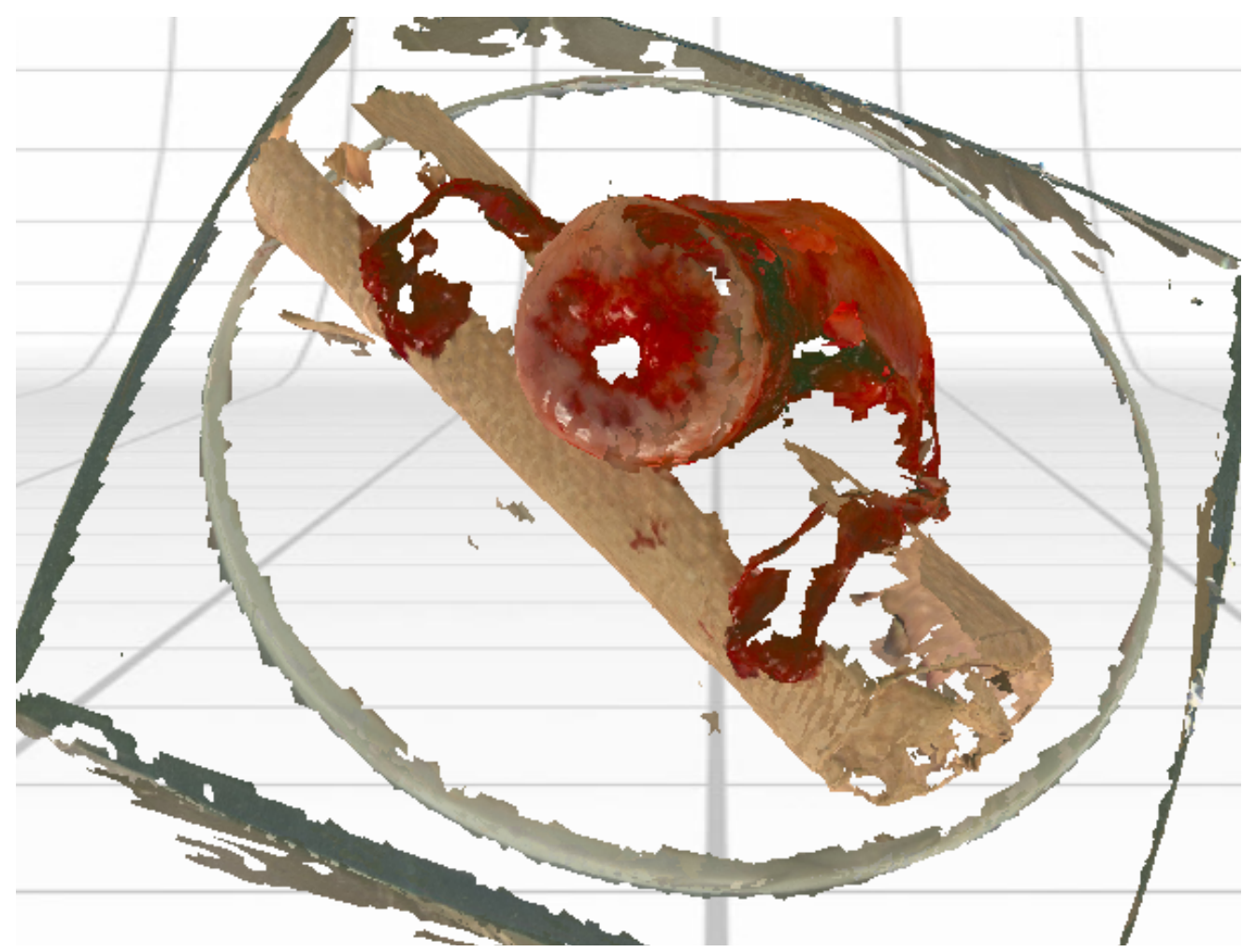

Figure A1: NextEngine 3D Scan of Cervix and Uterus 1

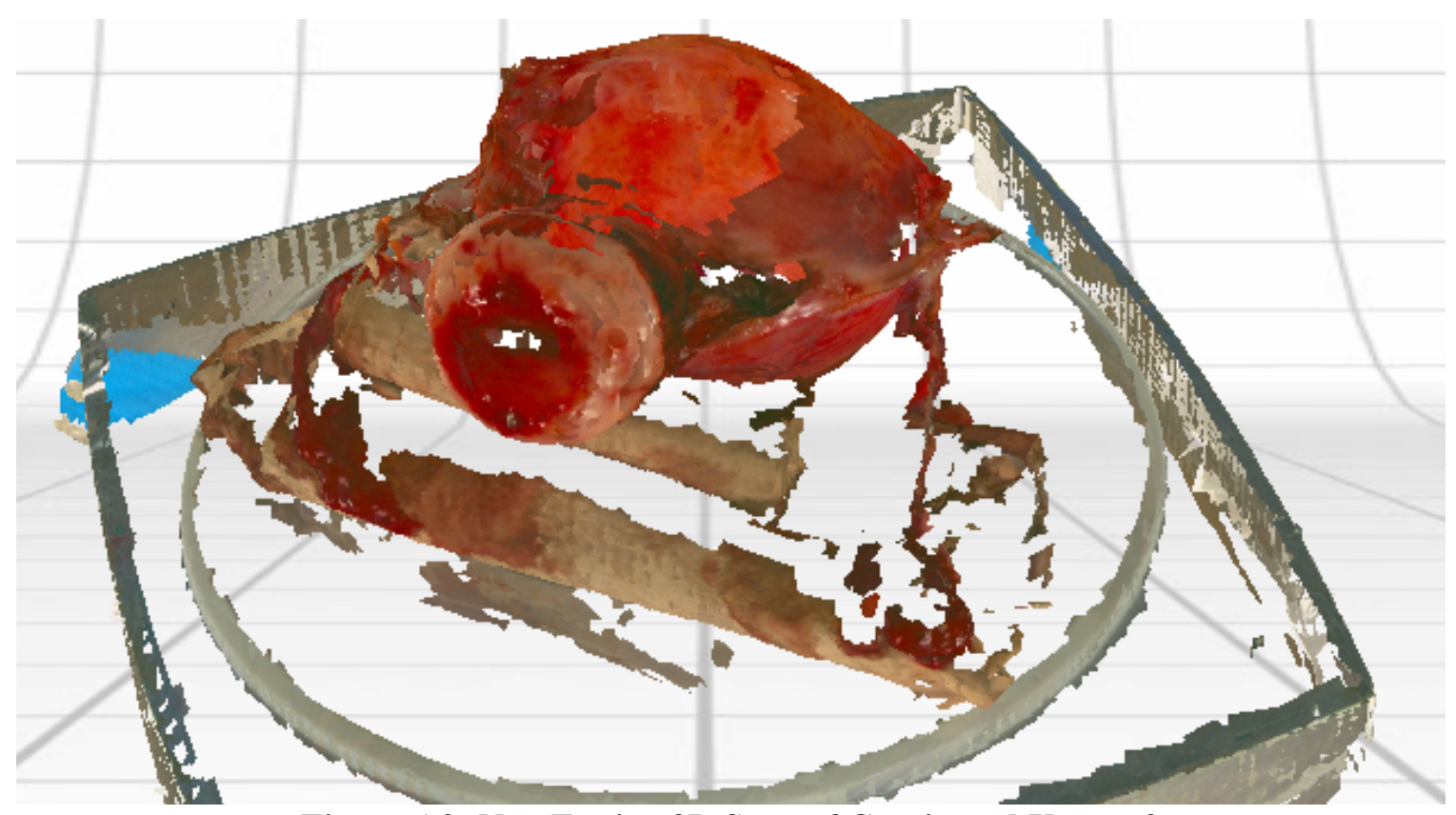

Figure A2: NextEngine 3D Scan of Cervix and Uterus 2 


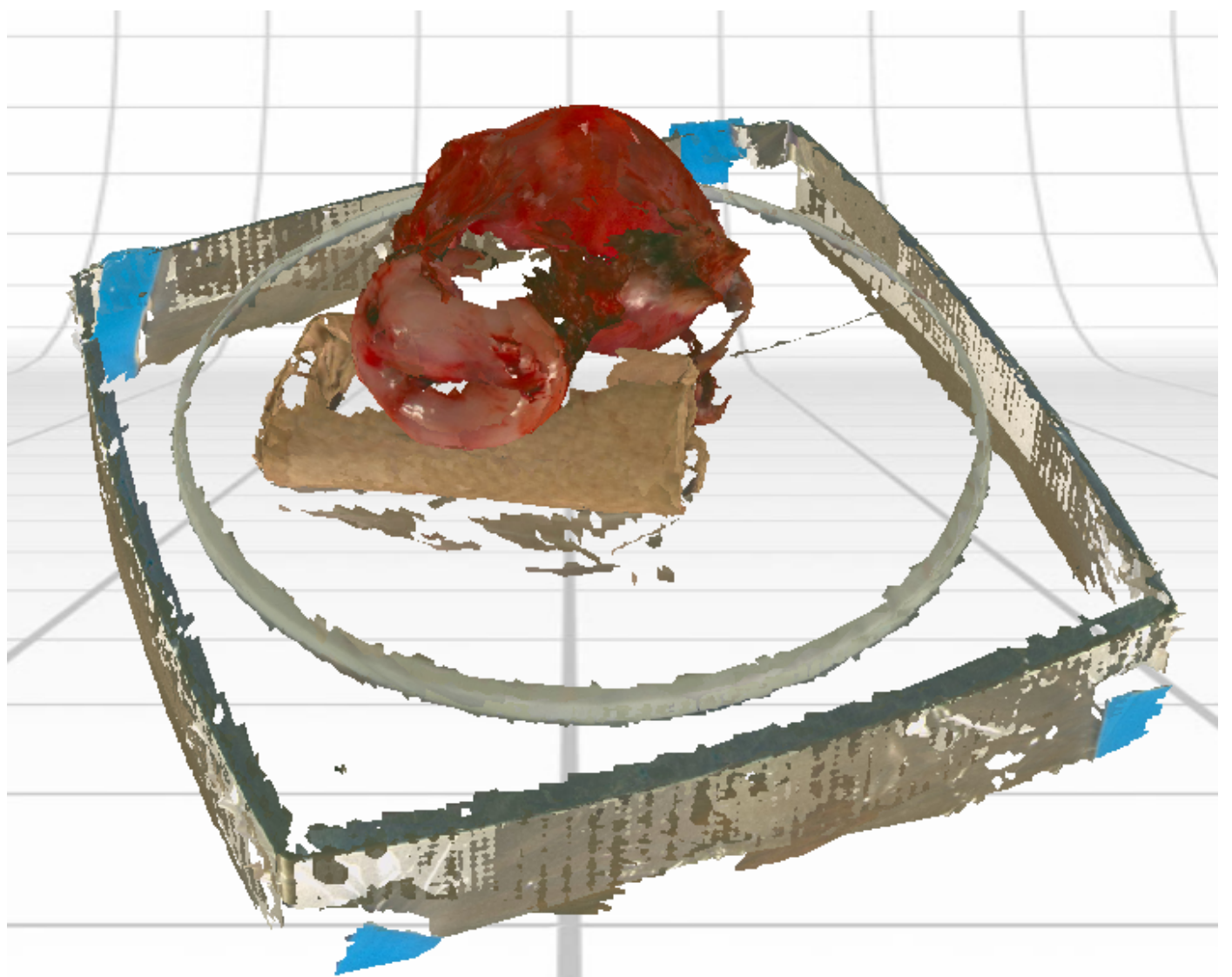

Figure A3: NextEngine 3D Scan of Cervix and Uterus 3

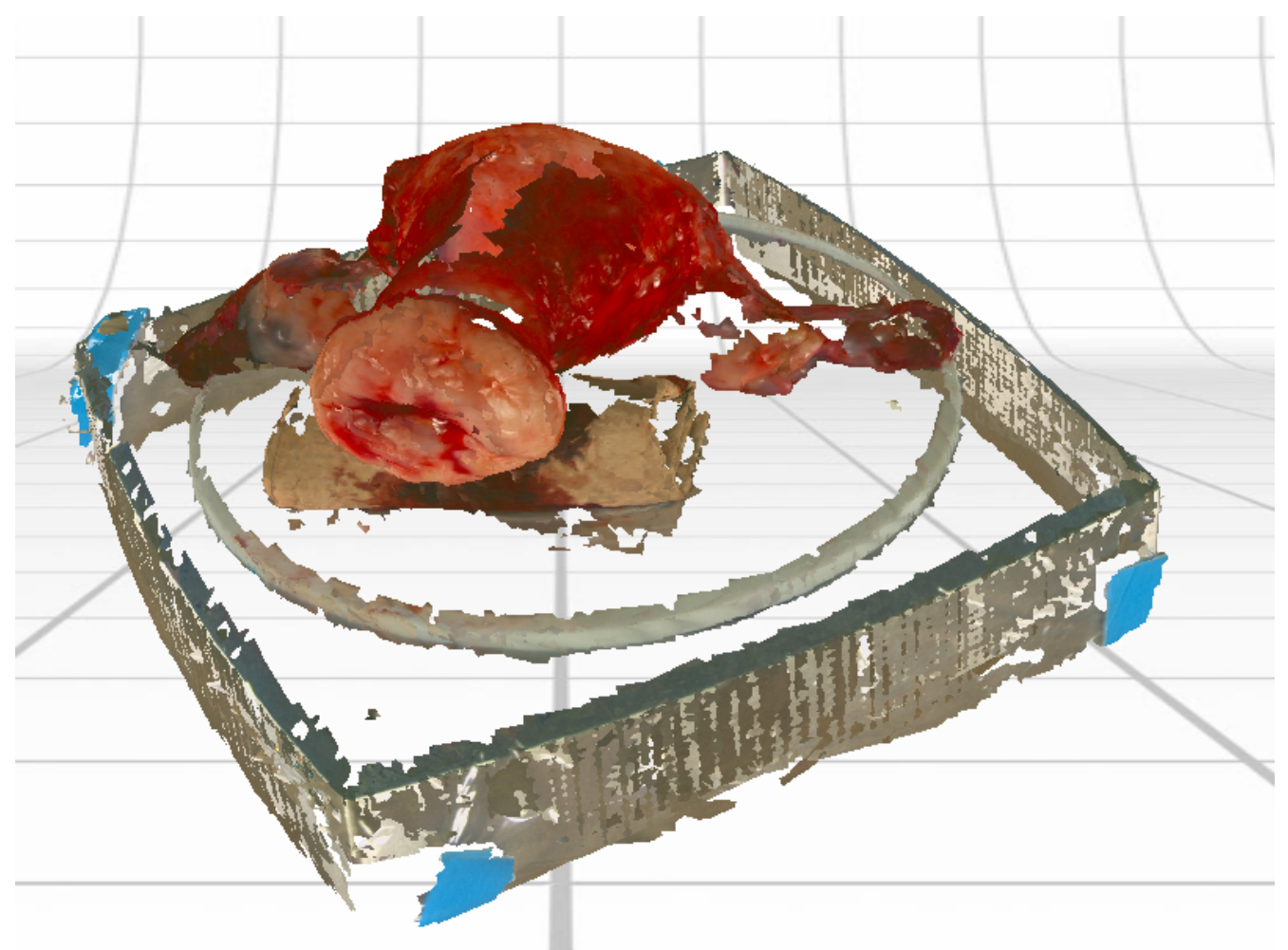

Figure A4: NextEngine 3D Scan of Cervix and Uterus 4 


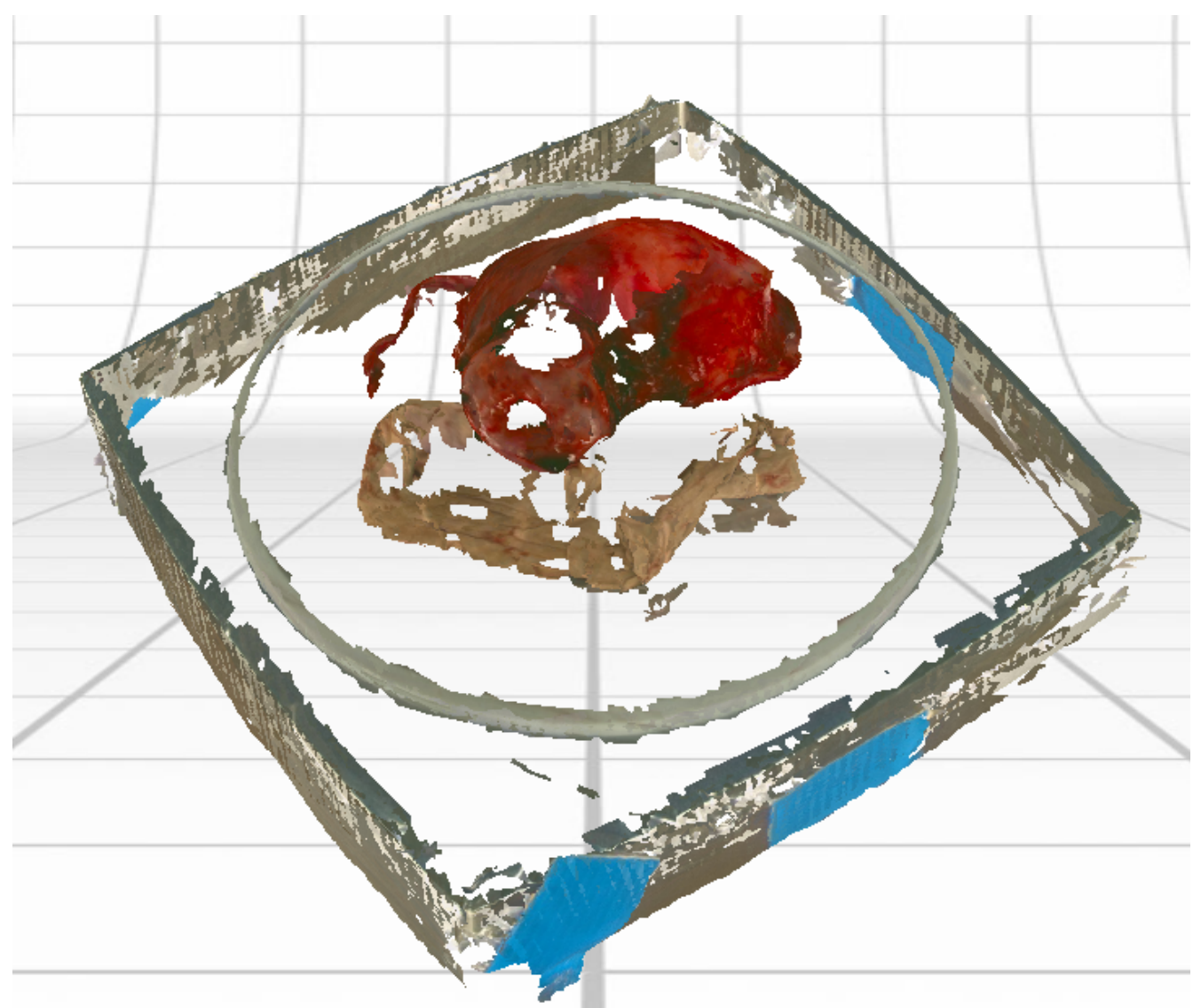

Figure A5: NextEngine 3D Scan of Cervix and Uterus 5

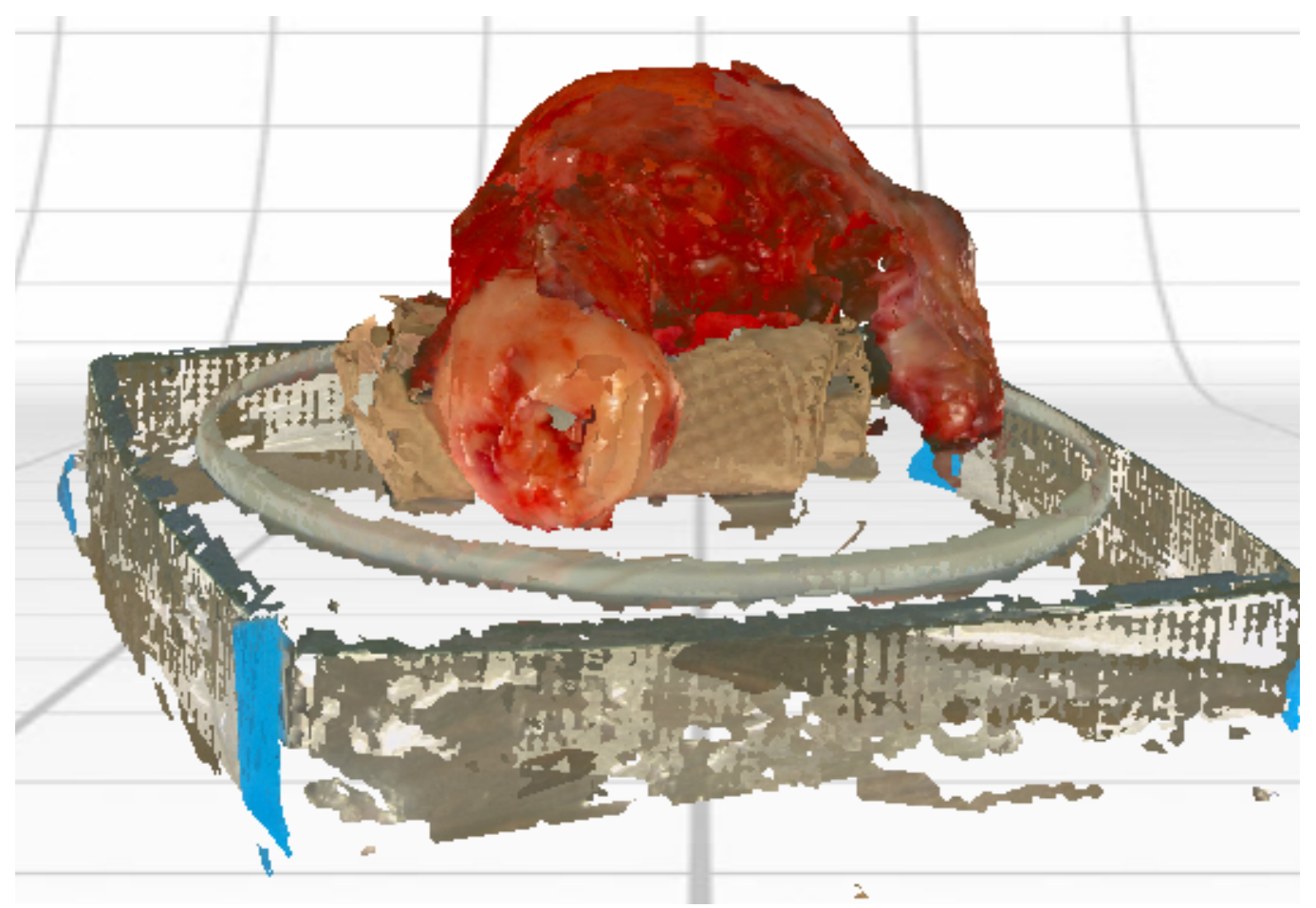

Figure A6: NextEngine Scan of Cervix and Uterus 6 


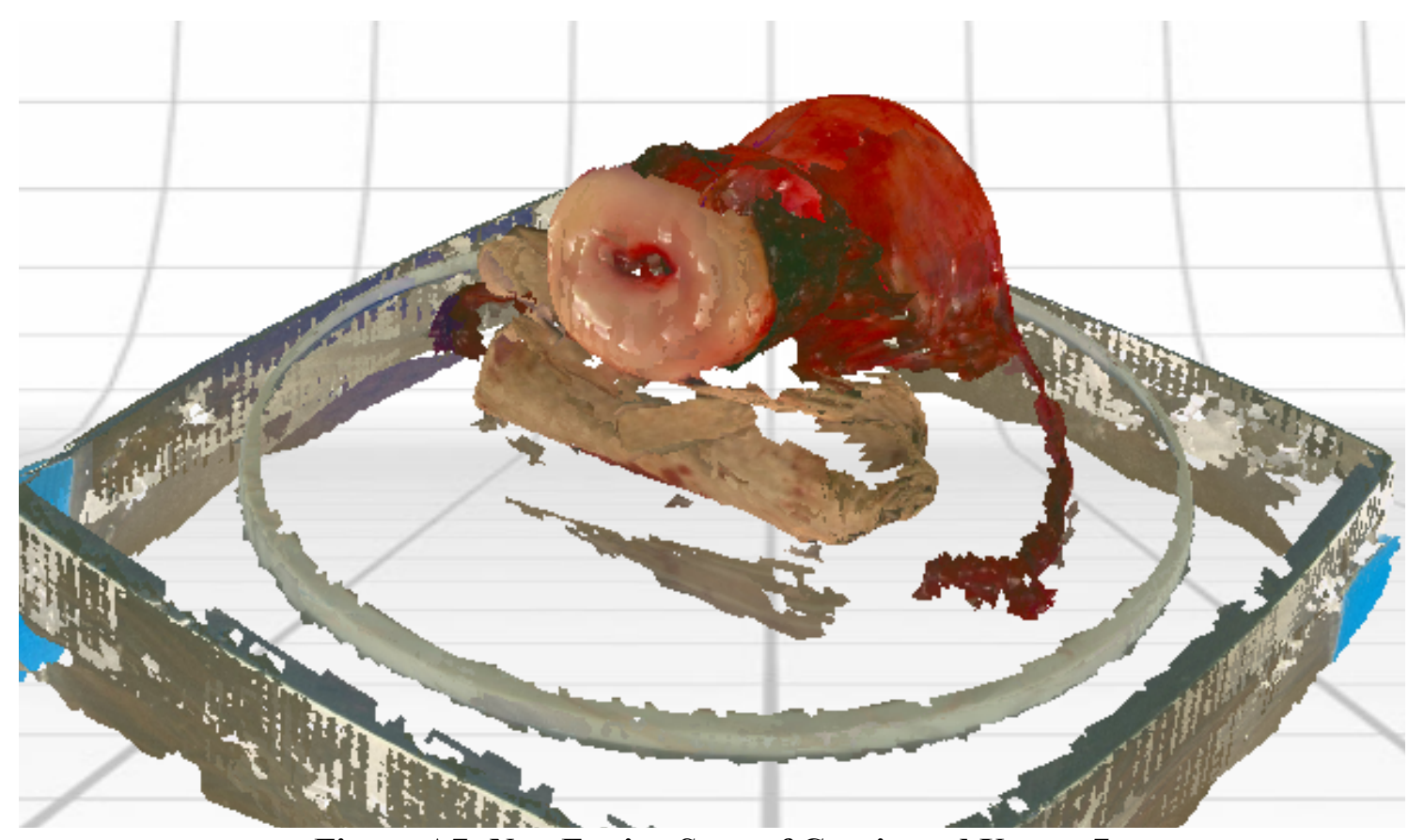

Figure A7: NextEngine Scan of Cervix and Uterus 7

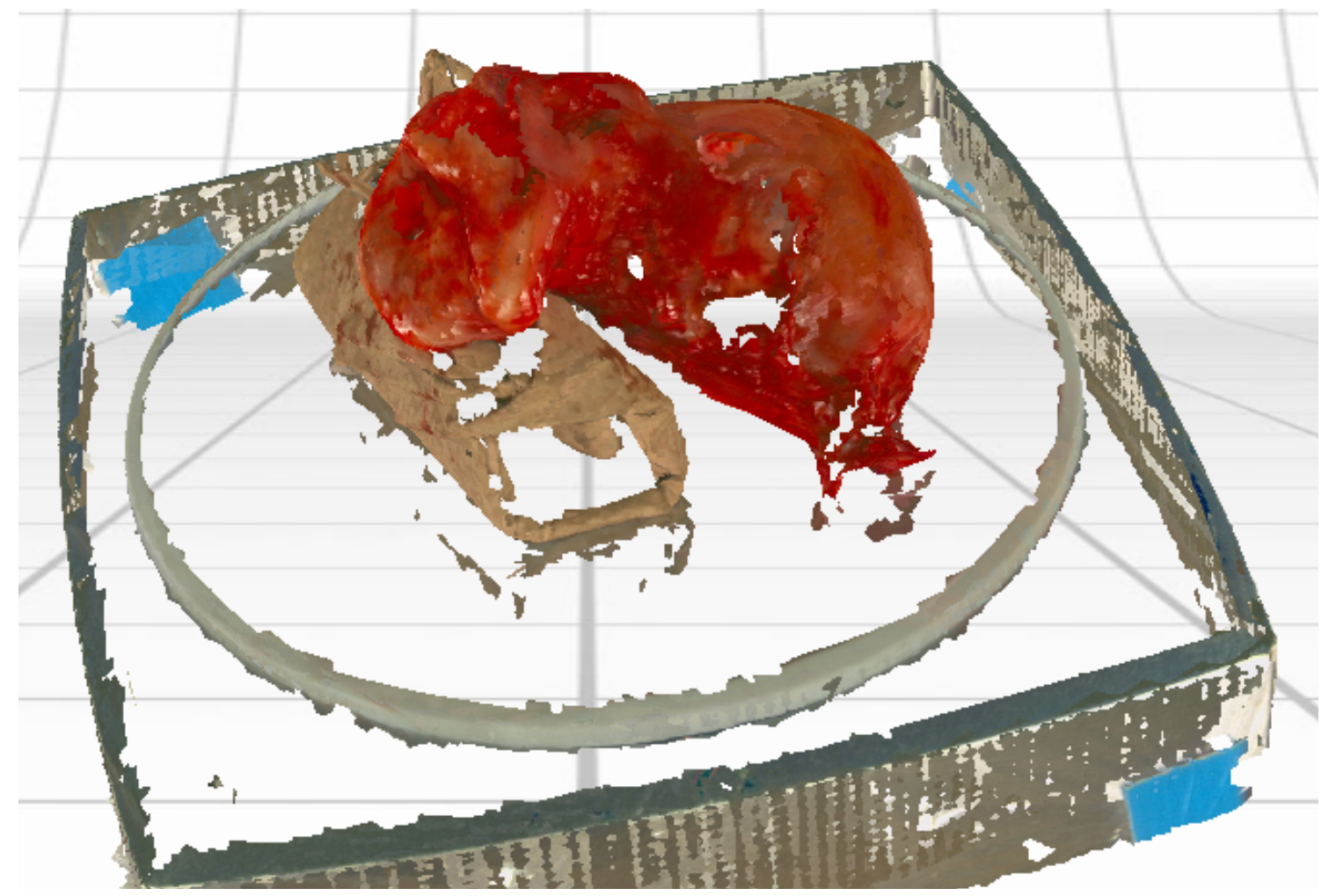

Figure A8: NextEngine Scan of Cervix and Uterus 8 


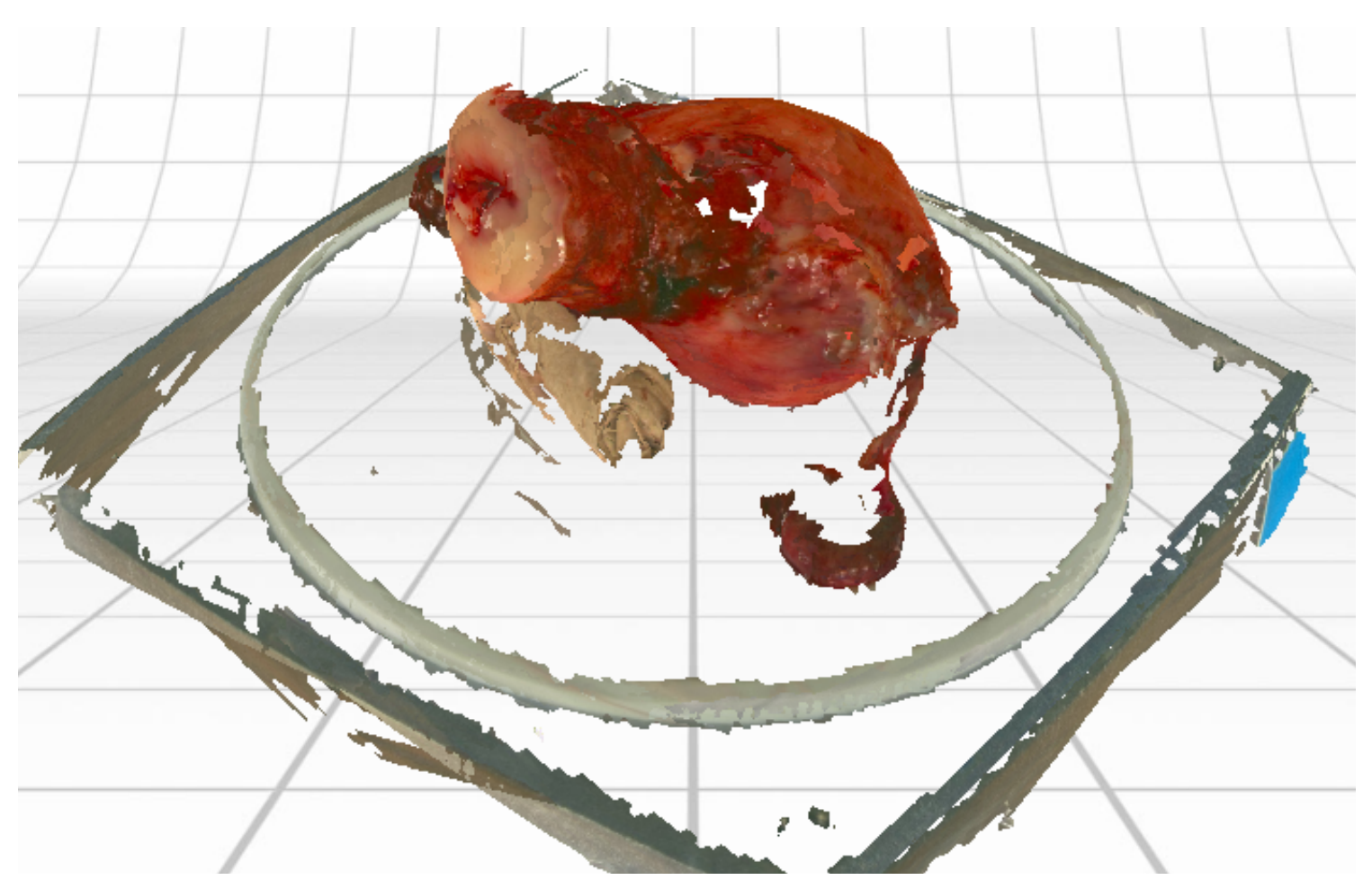

Figure A9: NextEngine Scan of Cervix and Uterus 9

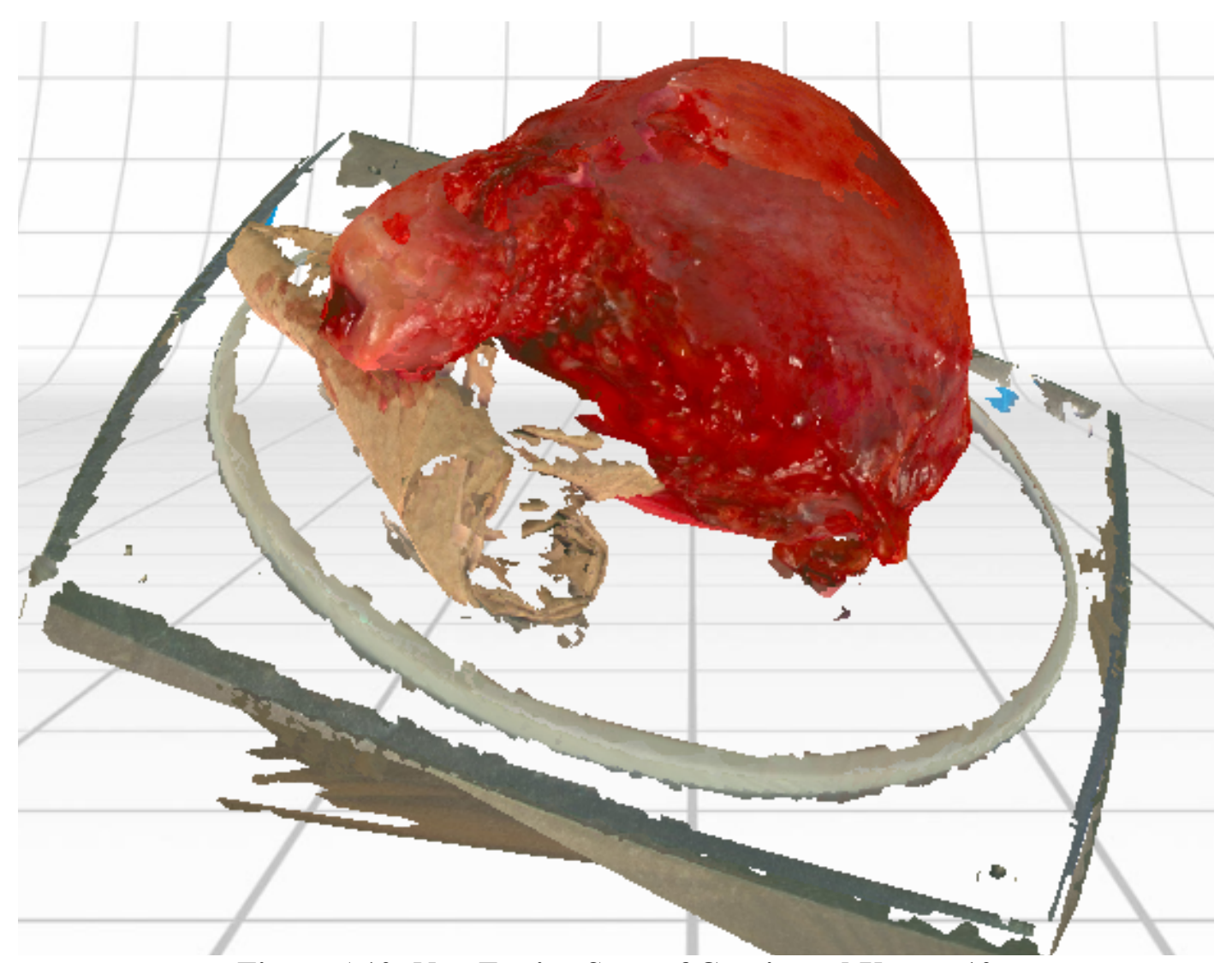

Figure A10: NextEngine Scan of Cervix and Uterus 10 


\section{References}

[1] S. Guendelman, D. Thornton, J. Gould, and N. Hosang, "Obstetric complications during labor and delivery: Assessing ethnic differences in California," Women's Health Issues, vol. 16, no. 4, pp. 189-197, 2006.

[2] A. H. Shennan, "Management of labour," Anaesthesia and Intensive Care Medicine, vol. 5, no. 7, pp. 226-227, Jul. 2004.

[3] B. Greulich and B. Tarrant, "The Latent Phase of Labor: Diagnosis and Management," Journal of Midwifery and Women's Health, vol. 52, no. 3, pp. 190-198, 2007.

[4] R. Garcia-Simon, D. Oros, D. Gracia-Colera, E. Moreno, C. Paules, S. Canizares, E. Gascon, and E. Fabre, "Cervix assessment for the management of labor induction: Reliability of cervical length and Bishop score determined by residents," Journal of Obstetrics and Gynaecology Research, vol. 41, no. 3, pp. 377-382, 2015.

[5] J. Iams, "Prediction and early detection of preterm labor," Obstetrics \& Gynecology, vol. 101, no. 2, pp. 402-412, 2003.

[6] D. C. Jude, G. G. Gilbert, and D. Magrane, "Simulation training in the obstetrics and gynecology clerkship," American Journal of Obstetrics and Gynecology, vol. 195, no. 5, pp. 1489-1492, 2006.

[7] A. G. Gallagher, E. M. Ritter, H. Champion, G. Higgins, M. P. Fried, G. Moses, C. D. Smith, and R. M. Satava, "Virtual reality simulation for the operating room: proficiencybased training as a paradigm shift in surgical skills training.," Annals of Surgery, vol. 241, no. 2, pp. 364-372, 2005.

[8] I. a. Grable and P. Ochoa, "Simulations in obstetrics," Disease-a-Month, vol. 57, no. 12, pp. 763-774, 2011.

[9] B. Robertson, "An obstetric simulation experience in an undergraduate nursing curriculum," Nurse Educator, vol. 31, no. 2, pp. 74-78, 2006.

[10] D. M. Gaba, "The future vision of simulation in health care.," Quality \& Safety in Healthcare, vol. 13 Suppl 1, pp. i2-i10, 2004.

[11] Next Engine Inc., "NextEngine 3D Scanner Ultra HD Tech Specs." [Online]. Available: http://www.nextengine.com/assets/pdf/scanner-techspecs-uhd.pdf. [May 30, 2016].

[12] S. Follmer, D. Leithinger, A. Olwal, N. Cheng, and H. Ishii, "Jamming User Interfaces: Programmable Particle Stiffness and Sensing for Malleable and Shape-Changing Devices," Proceedings of the 25th annual ACM symposium on User interface software and technology - UIST '12, pp. 519-528, 2012.

[13] M. B. Sennström, G. Ekman, G. Westergren-Thorsson, a Malmström, B. Byström, U. Endrésen, N. Mlambo, M. Norman, B. Ståbi, and a Brauner, "Human cervical ripening, an inflammatory process mediated by cytokines.," Molecular Human Reproduction, vol. 6, no. 4, pp. 375-381, 2000. 
[14] M. L. McCormick, H. C. G. Sanghvi, B. Kinzie, and N. McIntosh, "Preventing postpartum hemorrhage in low-resource settings," International Journal of Gynecology and

Obstetrics, vol. 77, no. 3, pp. 267-274, 2002.

[15] J. E. Norman, "Preterm labour. Cervical function and prematurity.," Clinical Obstetrics \& Gynaecology, vol. 21, no. 5, pp. 791-806, Oct. 2007.

[16] D. Danforth, "The morphology of the human cervix," Clinical Obstetrics \& Gynaecology., vol. 26, pp. 7-13, 1983.

[17] P. C. Leppert, “Anatomy and Physiology of Cervical Ripening," Clinical Obstetrics \& Gynaecology, vol. 38, no. 2, pp. 267-279, 1995.

[18] K. M. Myers, H. Feltovich, E. Mazza, J. Vink, M. Bajka, R. J. Wapner, T. J. Hall, and M. House, "The mechanical role of the cervix in pregnancy," Journal of Biomechanics, vol. 48, no. 9, pp. 1511-1523, 2015.

[19] S. Wozniak, P. Czuczwar, P. Szkodziak, and T. Paszkowski, "Usefulness of elastography in predicting the outcome of Foley catheter labour induction," Australian and New Zealand Journal of Obstetrics and Gynaecology, vol. 55, no. 3, pp. 245-250, 2015.

[20] E. H. Bishop, "Pelvic Scoring for Elective Induction," Obstetrics and Gynecology, vol. 24, no. 2, pp. 266-268, 1964.

[21] A. Cubal, J. Carvalho, M. J. Ferreira, G. Rodrigues, and O. Do Carmo, "Value of Bishop score and ultrasound cervical length measurement in the prediction of cesarean delivery.," Journal of Obstetrics and Gynaecology Research, vol. 39, no. 9, pp. 1391-6, Sep. 2013.

[22] E. F. Faltin-Traub, M. Boulvain, D. L. Faltin, P. Extermann, and O. Irion, "Reliability of the Bishop score before labour induction at term," European Journal of Obstetrics \& Gynecology and Reproductive Biology, vol. 112, no. 2, pp. 178-181, Feb. 2004.

[23] Z. Vaknin, Y. Kurzweil, and D. Sherman, "Foley catheter balloon vs locally applied prostaglandins for cervical ripening and labor induction: A systematic review and metaanalysis," American Journal of Obstetrics and Gynecology, vol. 203, no. 5, pp. 418429, 2010.

[24] J. E. Norman and S. Stock, "Intracervical Foley catheter for induction of labour," Lancet, vol. 378, no. 9809, pp. 2054-2055, 2011.

[25] M. Boulvain, C. Stan, and O. Irion, "Membrane sweeping for induction of labour.," Cochrane Database of Systematic Reviews, no. 1, p. CD000451, 2005.

[26] P. C. Tan, R. Jacob, and S. Z. Omar, "Membrane sweeping at the initiation of labour induction increased spontaneous vaginal delivery rates," Evidence-based Obstetrics \& Gynecology, vol. 8, no. 3-4, pp. 88-89, Sep. 2006.

[27] S. M. Yount and N. Lassiter, "The Pharmacology of Prostaglandins for Induction of Labor," Journal of Midwifery and Women's Health, vol. 58, no. 2, pp. 133-144, 2013.

[28] S. Niromanesh, A. Mosavi-Jarrahi, and F. Samkhaniani, "Intracervical Foley catheter balloon vs. prostaglandin in preinduction cervical ripening," International Journal of Gynecology and Obstetrics, vol. 81, no. 1, pp. 23-27, 2003. 
[29] M. Boulvain, a Kelly, C. Lohse, C. Stan, and O. Irion, "Mechanical methods for induction of labour.," Cochrane Database of Systematic Reviews, no. 4, p. CD001233, 2001.

[30] C. James, A. Peedicayil, and L. Seshadri, "Use of the Foley catheter as a cervical ripening agent prior to induction of labor," International Journal of Gynecology \& Obstetrics, vol. 47, no. 3, pp. 229-232, Dec. 1994.

[31] S. Deering, T. Auguste, and E. Lockrow, "Obstetric simulation for medical student, resident, and fellow education," Seminars in Perinatology, vol. 37, no. 3, pp. 143-145, 2013.

[32] S. Cooper, R. Cant, J. Porter, F. Bogossian, L. McKenna, S. Brady, and S. Fox-Young, "Simulation based learning in midwifery education: A systematic review," Women and Birth, vol. 25, no. 2, pp. 64-78, 2012.

[33] R. Hogan, "Simulation in midwifery education - what works? Findings from an extensive literature review," Women and Birth, vol. 24, p. S39, Oct. 2011.

[34] E. J. Kim, P. Theprungsirikul, M. K. McDonald, E. D. Gurewitsch, and R. H. Allen, “A biofidelic birthing simulator: Models for research and training in complicated human births," IEEE Engineering in Medicine and Biology Magazine, vol. 24, no. 6, pp. 34-39, 2004.

[35] A. Ziv, P. R. Wolpe, S. D. Small, and S. Glick, "Simulation-Based Medical Education: An Ethical Imperative," Academic Medicine, vol. 78, no. 8, pp. 783-788, 2003.

[36] S. W. Holmström, K. Downes, J. C. Mayer, and L. A. Learman, "Simulation training in an obstetric clerkship: a randomized controlled trial.," Obstetrics and Gynecology, vol. 118, no. 3, pp. 649-654, 2011.

[37] R. Gardner, "Simulation and simulator technology in obstetrics: past, present and future," Expert Review of Obstetrics \& Gynecology, vol. 2, p. 775, Jun. 2007.

[38] Limbs \& Things, "PROMPT Birthing Simulator Standard," 2007. [Online]. Available: http://assets.limbsandthings.com/uploads/80000_UG.pdf.

[39] Gaumard Scientific Inc., "Maternal and Neonatal Computer Interactive Simulation System With Maternal / Neonatal / Fetal Monitors." 2008. [Online]. Available: https://s3.amazonaws.com/gaumard2.0-downloads/manuals/36_S560.pdf [May 24, 2016].

[40] 3B Scientific GmbH, "SIMone: Obstetrical medicine background," 2008. [Online]. Available: https://www.a3bs.com/product-manual/XP818GMH_US09_screen.pdf.

[41] "CAE Healthcare presented its childbirth simulator for the first time in Canada," 2014. [Online] Available: http://www.cae.com/CAE-Healthcare-presented-its-childbirthsimulator-for-the-first-time-in-Canada-2014/?contextualBUID=178, [June 13, 2016].

[42] C. H. S.L.York, S.Migas, J.Haag, Z.Liu, R.Stiller, W.C. McGaghie, "Creation and Initial Assessment of a Second-Trimester Uterine Model," Simulation in Healthcare, vol. 9, no. 3, pp. 199-202, 2014.

[43] J. S. Kim, "3D printed phantom to mimic dynamic softening of cervix during pre-labour," M.A.Sc thesis, Ryerson University, Canada, 2015.

[44] S. Kim, C. Laschi, and B. Trimmer, "Soft robotics: A bioinspired evolution in robotics," Trends in Biotechnology, vol. 31, no. 5, pp. 287-294, 2013.

[45] Z. Wang, M. Z. Q. Chen, and J. Yi, "Soft robotics for engineers," HKIE Transactions, vol. 22, no. 2, pp. 88-97, 2015. 
[46] L. Shi, S. Guo, M. Li, S. Mao, N. Xiao, B. Gao, Z. Song, and K. Asaka, "A novel soft biomimetic microrobot with two motion attitudes.," Sensors (Basel, Switzerland)., vol. 12, no. 12, pp. 16732-58, Jan. 2012.

[47] F. Ilievski, A. D. Mazzeo, R. F. Shepherd, X. Chen, and G. M. Whitesides, "Soft robotics for chemists," Angewandte Chemie - International Edition, vol. 50, no. 8, pp. 1890-1895, 2011.

[48] H. Lee, C. Xia, and N. X. Fang, "First jump of microgel; actuation speed enhancement by elastic instability," Soft Matter, vol. 6, no. 18, p. 4342, Sep. 2010.

[49] N. G. Cheng, M. B. Lobovsky, S. J. Keating, A. M. Setapen, K. I. Gero, A. E. Hosoi, and K. D. Iagnemma, "Design and analysis of a robust, low-cost, highly articulated manipulator enabled by jamming of granular media," Proceedings - IEEE International Conference on Robotics and Automation, pp. 4328-4333, 2012.

[50] J. Amend, E. Brown, N. Rodenberg, H. Jaeger, and H. Lipson, "A Positive Pressure Universal Gripper Based on the Jamming of Granular Material," IEEE Transactions on Robotics, vol. 28, no. 2, pp. 341-350, 2012.

[51] E. Brown, N. Rodenberg, J. Amend, A. Mozeika, E. Steltz, M. R. Zakin, H. Lipson, and H. M. Jaeger, "Universal robotic gripper based on the jamming of granular material," Proceedings of the National Academy of Sciences, vol. 107, no. 44, pp. 18809-18814, Oct. 2010.

[52] S. Mishra, "Application of 3D printing in medicine," Indian Heart Journal, vol. 68, no. 1, pp. $108-109,2016$.

[53] Z. Wang, M. Z. Q. Chen, and J. Yi, "Soft robotics for engineers," HKIE Transactions, vol. 22, no. 2, pp. 88-97, May 2015.

[54] N. W. Bartlett, M. T. Tolley, J. T. B. Overvelde, J. C. Weaver, B. Mosadegh, K. Bertoldi, G. M. Whitesides, and R. J. Wood, "Robot Powered By Combustion," Science, vol. 349, no. July, pp. 161-165, 2015.

[55] Internet Wire, "NinjaFlex(R) 3D Printer Filament Makes Waves," Business Insights: Global, Jul-2014. [Online]. Available:

http://bi.galegroup.com.ezproxy.lib.ryerson.ca/global/article/GALE\%7CA375036896/9a4 51a7a6f9c5b8cbb62651060f320e8?u=rpu_main [May 12, 2016].

[56] S. Desai, T. Sinha, and A. Mahal, "Prevalence of hysterectomy among rural and urban women with and without health insurance in Gujarat, India," Reproductive Health Matters, vol. 19, no. 37, pp. 42-51, 2011.

[57] K. M. Myers, A. P. Paskaleva, M. House, and S. Socrate, "Mechanical and biochemical properties of human cervical tissue," Acta Biomaterialia, vol. 4, no. 1, pp. 104-116, 2008.

[58] W. Yao, K. Yoshida, M. Fernandez, J. Vink, R. J. Wapner, C. V. Ananth, M. L. Oyen, and K. M. Myers, "Measuring the compressive viscoelastic mechanical properties of human cervical tissue using indentation," Journal of the Mechanical Behavior of Biomedical Materials, vol. 34, pp. 18-26, 2014.

[59] R. J. Dewall, T. Varghese, M. a Kliewer, J. M. Harter, and M. Hartenbach, "CompressionDependent Viscoelastic Behavior of Human Cervix Tissue," Ultrasonic Imaging, vol. 32, no. 4, pp. 214-228, 2010.

[60] N. Jagani, H. Schulman, A. Fleischer, J. Mitchell, and G. Randolph, "Role of the cervix in the induction of labor.," Obstetrics and Gynecology, vol. 59, no. 1, pp. 21-26, 1982.

[61] J. Smith, "A simplified cervix model in response to induction balloon in pre-labour," Theoretical Biology and Medical Modelling, vol. 10, no. 1, p. 58, 2013. 
[62] Aleph Objects, "TAZ 4 User Manual," 2014. [Online]. Available: https://download.lulzbot.com/TAZ/4.0/documentation/2014Q2/manual/TAZ_4_Manual.p df. [May 19, 2016].

[63] C. Purdel and M. Stere, "Stress analysis of toroidal shell," INCAS Bulletin, vol. 2, no. 4, pp. 215-224, 2010.

[64] J. Iams, R. B. Newman, E. A. Thom, and R. L. Goldenberg, "Frequency of uterine contractions and the risk of spontaneous preterm delivery," New England Journal of Medicine, vol. 346, no. 4, pp. 250-256, 2002.

[65] J. A. Smith, A. Leshchenko, K. Atwell, and A. El-Falou, "A Dynamic Cervical Model for Training and Induction Device Testing," Daphne Cockwell School of Nursing Sixth Annual Research Day. 2013.

[66] Structur3d Printing, "Discov3ry: A Universal Paste Extruder for YOur Desktop 3D Printer," 2016. [Online]. Available: http://www.structur3d.io/discov3ryproducts/discov3ry. [June 2, 2016]. 


\section{Glossary}

cervical os- the lower part of the cervical canal opening into the vagina from the uterus

cervical ripening- process that occurs at the onset of labour that softens the cervix in preparation for dilation

dilation- opening of the cervical canal such that the newborn's head can pass through during labour

effacement- the thinning out of the cervical length during labour

extracellular matrix- collection of molecules around cells that provide structural and biochemical support

fetal membrane rupture- breaking of the amniotic sac, commonly referred to as the water breaking

latent labour- the initial phase of labour, characterized by the softening of the cervix and dilation up to $3 \mathrm{~cm}$

uterine contractility- the ability of the uterine smooth muscle to contract to allow for dilation and effacement

uterus liquor- the amniotic fluid, or commonly referred to as the pregnant woman's water 